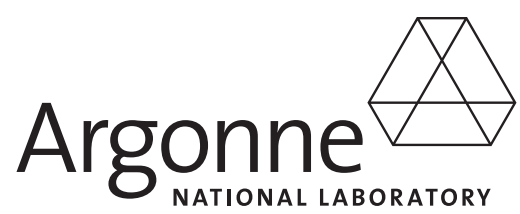

ANL/EVS/AGEM/TR-06-08

\title{
Final Work Plan for Targeted Investigation at Hilton, Kansas
}

\section{Environmental Science Division}




\begin{abstract}
About Argonne National Laboratory
Argonne is a U.S. Department of Energy laboratory managed by UChicago Argonne, LLC under contract DE-AC02-06CH11357. The Laboratory's main facility is outside Chicago, at 9700 South Cass Avenue, Argonne, Illinois 60439. For information about Argonne, see www.anl.gov.
\end{abstract}

\title{
Availability of This Report
}

This report is available, at no cost, at http://www.osti.gov/bridge. It is also available on paper to the U.S. Department of Energy and its contractors, for a processing fee, from:

U.S. Department of Energy

Office of Scientific and Technical Information

P.O. Box 62

Oak Ridge, TN 37831-0062

phone (865) 576-8401

fax (865) 576-5728

reports@adonis.osti.gov

\section{Disclaimer}

This report was prepared as an account of work sponsored by an agency of the United States Government. Reference herein to any specific commercial product, process, or service by trade name, trademark, manufacturer, or otherwise, does not necessarily constitute or imply its endorsement, recommendation, or favoring by the United States Government or any agency thereof. The views and opinions of document authors expressed herein do not necessarily state or reflect those of the United States Government or any agency thereof, Argonne National Laboratory, or UChicago Argonne, LLC. 


\section{Final Work Plan for Targeted Investigation at Hilton, Kansas}

by

Applied Geosciences and Environmental Management Section

Environmental Science Division, Argonne National Laboratory

June 2007

USDA 


\section{Contents}

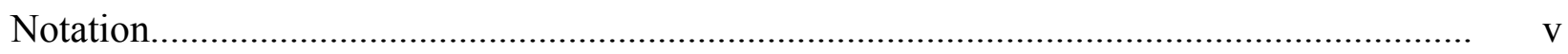

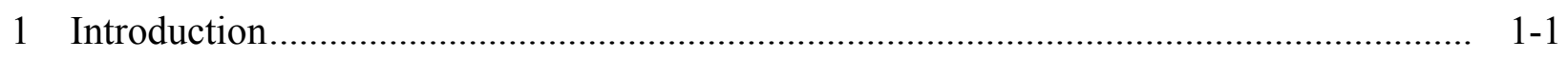

2 Background Information and Previous Studies ................................................................ 2-1

$2.1 \quad$ Background Information............................................................................... 2-1

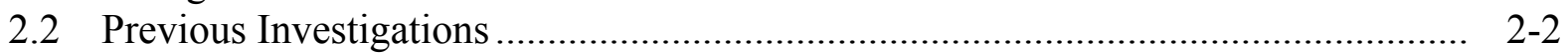

2.2.1 Geologic and Hydrogeologic Setting ........................................................ 2-2

2.2.2 Groundwater and Soil Contamination ........................................................ 2-4

2.3 Identification of the Former Grain Storage Facilities ............................................... 2-5

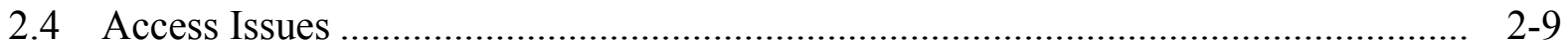

3 Technical Program ............................................................................................ $3-1$

3.1 Project Objectives ........................................................................................ 3-1

3.2 Scope of Work ………………...................................................................... 3-1

3.2.1 Segment 1: Investigate for Carbon Tetrachloride Contamination in

Shallower Soil and the Shallow Aquifer Unit on the Former CCC/USDA

Property......

3.2.2 Segment 2: If Contamination Is Found in Shallower Soil and/or the Shallow Aquifer Unit on the Former CCC/USDA Property, Extend the Investigation for Carbon Tetrachloride Contamination Downward to the Main Aquifer Unit, and Determine the Groundwater Flow Direction.

3.2.3 Segment 3: If a Significant Contaminant Source Is Confirmed on the Former CCC/USDA Property, Determine the Lateral and Vertical Extent of the Carbon Tetrachloride Plume Emanating from the Property ................ 3-6

3.2.4 Segment 4: If the Former CCC/USDA Facility Is Identified as a Contaminant Source, Establish a Groundwater Monitoring Network ........... 3-7

3.3 Investigation Methods...................................................................................... 3-7

3.3.1 Methods for Sampling of Existing Wells..................................................... 3-7

3.3.2 Methods for Groundwater Sampling with the Cone Penetrometer................ 3-8

3.3.3 Methods for Vertical-Profile Soil Sampling with the Cone Penetrometer .... 3-9

3.3.4 Methods for Soil and Groundwater Sampling with the Sonic Drilling Rig ............................................................................................. 3-10

3.3.5 Methods for Installing Permanent Piezometers and Monitoring Wells......... 3-10

3.3.6 Methods for Handling and Disposal of Investigation-Derived Waste........... 3-11

3.4 Sampling and Reporting Schedule ...................................................................... 3-12

3.5 Quality Assurance and Quality Control................................................................... 3-13

3.6 Health and Safety Information......................................................................... 3-15

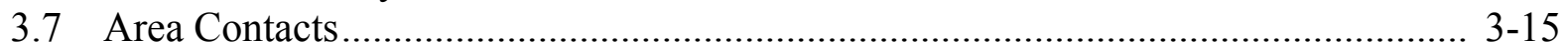

3.8 Argonne Contacts............................................................................................. 3-16

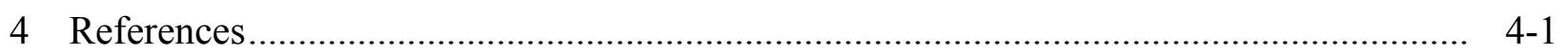


Appendix A: Comprehensive Historical Analytical Data for Hilton, Kansas ........................ A-1

Appendix B: Records of Property Transactions Affecting the Grain Storage Facilities at Hilton, Kansas

\section{Figures}

1.1 Locations of Hilton, Kansas, the former CCC/USDA and private grain storage facilities, and contaminated well GW01

2.1 Historical results of carbon tetrachloride analyses on groundwater samples collected in the Hilton area

2.2 Simplified stratigraphic section derived from core descriptions for SB01, SB06, and SB07 at Hilton.

2.3 Locations of monitoring wells, piezometers, and geologic cross sections

2.4 Southeast-to-northwest geologic cross section $\mathrm{A}-\mathrm{A}^{\prime}$, illustrating the stratigraphic relationships in the vicinity of the former CCC/USDA and private grain storage facilities at Hilton

2.5 West-to-east geologic cross section B-B', illustrating the stratigraphic relationships in the vicinity of the former $\mathrm{CCC} / \mathrm{USDA}$ and private grain storage facilities at Hilton.

2.6 Regional potentiometric surface for the Equus Beds aquifer, McPherson County, Kansas, prepared from Kansas Geological Survey records and Argonne Phase II water level data

2.7 Soil and vegetation sampling locations (1996) and analytical results for carbon tetrachloride, shown on a 1963 aerial photograph

2.8 Locations of current property boundaries, the former CCC/USDA facility, the present Mid-Kansas Co-op, and the former Union Pacific Railroad Company property at Hilton

2.9 The maximum scale of grain storage facilities at Kansas, interpreted from a 1963 aerial photograph

3.1 Proposed investigation locations on the former CCC/USDA property 3-18

3.2 Interpreted hydrologic section B-B' through the former CCC/USDA grain storage facility 
Work Plan for Targeted Investigation at Hilton, Kansas

Version 03, 05/17/07

\section{Figures (cont.)}

3.3 Locations of existing monitoring wells, piezometers, and well GW01 .................. 3-20

3.4 Emergency route from Hilton to Memorial Hospital, McPherson, Kansas ................. 3-21

\section{Tables}

3.1 Construction information for existing wells and piezometers at Hilton, Kansas ......... 3-5

3.2 Emergency information for the Hilton, Kansas, investigation ............................... 3-17

A.1 Analytical results for previous sampling at Hilton, Kansas ................................... A-2

B.1 Timeline of Hilton property documentation on file at Argonne ............................. B-2

C.1 Potential applicable or relevant and appropriate requirements for

former CCC/USDA facilities in Kansas ............................................................. C-9 


\section{Notation}

AGEM Applied Geosciences and Environmental Management

AMSL above mean sea level

AOC administrative order on consent

ASCS Agricultural Stabilization and Conservation Service

BGL below ground level

${ }^{\circ} \mathrm{C} \quad$ degree(s) Celsius

CCC Commodity Credit Corporation

CPT cone penetrometer

EPA U.S. Environmental Protection Agency

$\mathrm{ft} \quad$ foot (feet)

gal gallon(s)

gpm gallon(s) per minute

h hour

I.D. inner diameter

IDW investigation-derived waste

in. inch(es)

KDHE Kansas Department of Health and Environment

KGS Kansas Geological Survey

$\mu \mathrm{g} / \mathrm{kg} \quad$ microgram(s) per kilogram

$\mu \mathrm{g} / \mathrm{L} \quad$ microgram(s) per liter

$\mathrm{mg} / \mathrm{L} \quad$ milligram(s) per liter

mi mile(s)

NAIP National Agricultural Imagery Program

$\mathrm{pg} / \mathrm{g} \quad$ picogram(s) per gram

PVC polyvinyl chloride

QA quality assurance

QC quality control

USDA U.S. Department of Agriculture

VOC volatile organic compound 


\section{Final Work Plan for Targeted Investigation at Hilton, Kansas}

\section{Introduction}

This Work Plan outlines the scope of a targeted investigation to update the status of carbon tetrachloride contamination in groundwater associated with grain storage operations at Hilton, Kansas. The Commodity Credit Corporation (CCC), an agency of the U.S. Department of Agriculture (USDA), operated a grain storage facility in Hilton during the 1950s and 1960s. At the time of the CCC/USDA operation in Hilton, grain storage facilities (CCC/USDA and private) were located along the both sides of the former Union Pacific railroad tracks (Figure 1.1). The main grain storage structures were on or near the railroad right-of-way.

The proposed targeted investigation, to be conducted by Argonne National Laboratory on the behalf of CCC/USDA, will supplement Argonne's Phase I and Phase II investigations in 1996-1997. The earlier investigations erroneously focused on an area east of the railroad property where the CCC/USDA did not operate, specifically on a private grain storage facility. In addition, the investigation was limited in scope, because access to railroad property was denied (Argonne 1997a,b). The hydrogeologic system at Hilton is potentially complex.

In 2005, Argonne conducted a property records search on behalf of the CCC/USDA. The documents found indicate the following:

- The CCC/USDA leased only one strip of land, on the west side of the former railroad tracks, from 1954 to 1965, for use as a grain storage facility. The grain storage facilities on the east side of the railroad tracks were operated by various commercial entities.

- The property formerly owned by Union Pacific Railroad Company was conveyed to the Central Kansas Conservancy in 1997. The railroad tracks were abandoned, and the bed is now used as a walking and biking trail.

On the basis of the results of the 2005 property records search and Argonne's 1996-1997 Phase I and Phase II investigations, and in response to a request of the Kansas Department of Health and Environment (KDHE), the following specific technical objectives are proposed for this targeted investigation: 
- Investigate for carbon tetrachloride contamination in shallower soil and the shallow aquifer unit on the former CCC/USDA property (west of the former railroad) to determine whether a soil source of carbon tetrachloride and a soilto-groundwater migration pathway exist on the property.

- If contamination is found in shallower soil and/or the shallow aquifer unit on the former CCC/USDA property, extend the investigation for carbon tetrachloride contamination to the main aquifer unit, and determine the groundwater flow direction.

- If a significant contaminant source is found on the former CCC/USDA property, determine the lateral and vertical extent of the carbon tetrachloride plume emanating from the property.

- If the former CCC/USDA facility is identified as a contaminant source, establish a groundwater monitoring network.

The results of the proposed work will provide the basis for determining what further CCC/USDA activities are necessary at the Hilton site.

To ensure the involvement of the CCC/USDA and KDHE project managers in decision making during the Hilton investigation, a stepwise approach is proposed. The proposed investigation includes the following four segments, in chronological order:

- Segment 1: Investigate for carbon tetrachloride contamination in shallower soil and the shallow aquifer unit on the former CCC/USDA property (west of the former railroad) to determine whether a soil source of carbon tetrachloride and a soil-to-groundwater migration pathway exist on the property.

- Activity 1: At five proposed locations on the former CCC/USDA property, use the cone penetrometer (CPT) unit to collect subsurface soil samples in vertical profiles at intervals of $4 \mathrm{ft}$ or at changes in lithology, to the depth of refusal. If the shallow aquifer unit is encountered (at a depth of approximately $80 \mathrm{ft}$ BGL [below ground level]), collect groundwater samples, install temporary piezometers, and determine the groundwater 
flow direction. Analyze the soil samples for volatile organic compounds (VOCs); analyze the groundwater samples for VOCs and 1,2-dibromoethane.

- Segment 2: If contamination is found in shallower soil and/or the shallow aquifer unit on the former CCC/USDA property, extend the investigation for carbon tetrachloride contamination downward to the main aquifer unit, and determine the groundwater flow direction.

- Activity 2a: To determine whether the former CCC/USDA facility contributed to the contamination identified in the lower aquifer at well GW01, use the CPT (or a sonic drilling rig if necessary) to collect soil samples in vertical profiles at the locations investigated in Segment 1 at which carbon tetrachloride was found. When the main aquifer is encountered (at a depth of approximately $170 \mathrm{ft}$ BGL), collect groundwater samples. Analyze the soil samples for volatile organic compounds (VOCs); analyze the groundwater samples for VOCs and 1,2-dibromoethane.

- Activity 2b: For groundwater level measurements, install temporary piezometers (if CPT installations are possible) or permanent piezometers (if drilling is required) at appropriate locations on the former CCC/USDA property investigated in Activity 2a. Measure groundwater levels in the available wells and piezometers.

- Activity 2c: Acquire detailed information, both in the field and from available records, regarding current and historical water usage and pumping rates from irrigation wells in the immediate vicinity of the Hilton site, to evaluate their potential to significantly affect the local hydrology.

- Segment 3: If a significant contaminant source is confirmed on the former CCC/USDA property, determine the lateral and vertical extent of the carbon tetrachloride plume emanating from the property. 
- Activity 3a: Conduct groundwater sampling for VOCs and 1,2-dibromoethane analyses in existing monitoring wells and piezometers determined to be downgradient, as well as in selected upgradient wells.

- Activity 3b: Conduct additional groundwater sampling for VOCs and 1,2-dibromoethane analyses at selected new upgradient and downgradient locations, through use of the CPT if possible.

- Segment 4: If the former CCC/USDA facility is identified as a contaminant source, establish a groundwater monitoring network.

- Activity 4: Install permanent piezometers or monitoring wells at selected locations, both upgradient and downgradient from the former CCC/USDA facility, to form a network for monitoring of contaminant migration and seasonal groundwater level fluctuations, as well as for determination of the potentially significant effects of pumping from nearby irrigation wells on the groundwater flow pattern and contaminant migration pathways. Locations available for the installations will depend on the success of access negotiations.

The CCC/USDA and KDHE project managers will be informed of all results and will jointly evaluate (1) the need to progress to the next segment of the investigation and (2) alterations to the existing Work Plan that are required as a consequence of the initial results. 


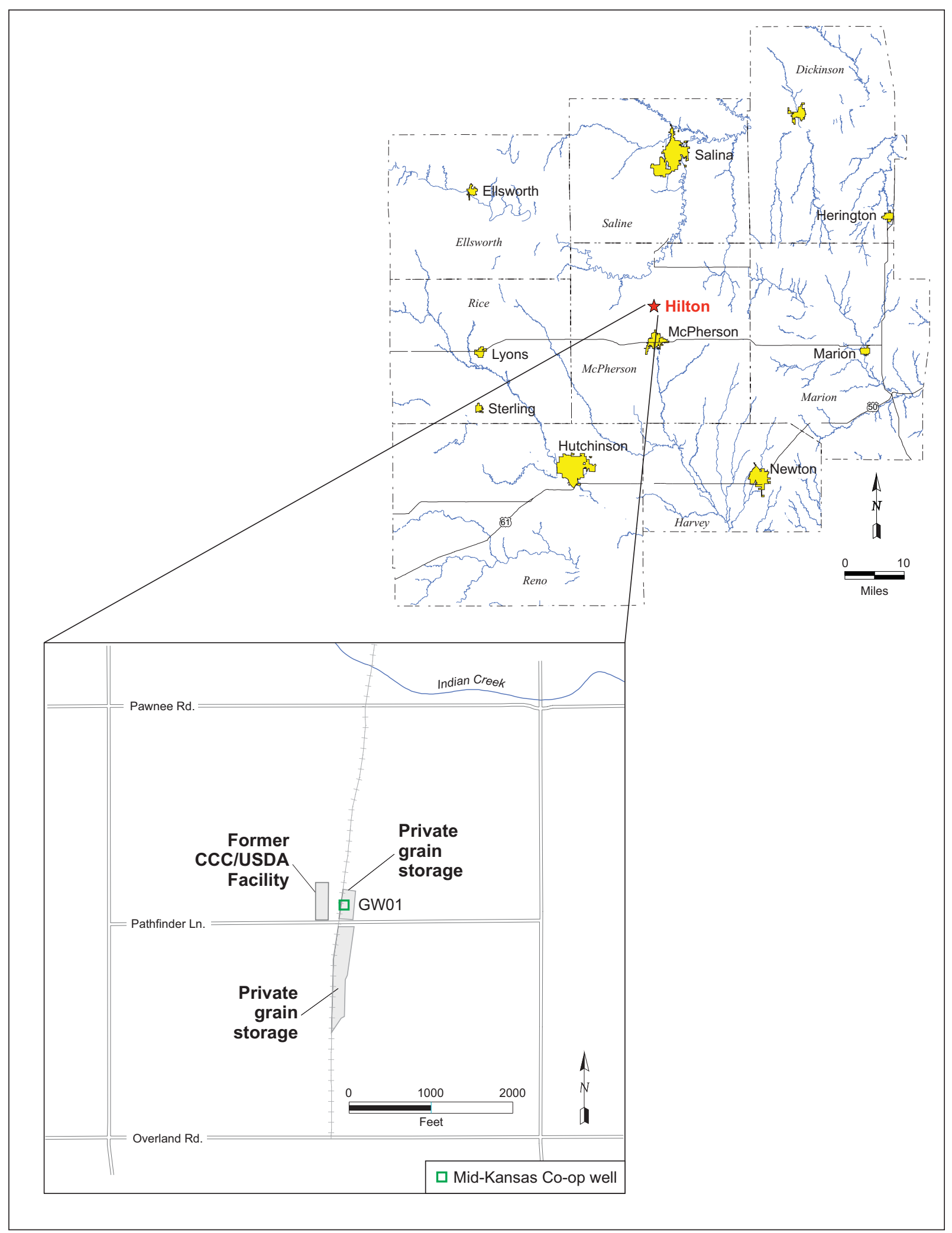

FIGURE 1.1 Locations of Hilton, Kansas, the former CCC/USDA and private grain storage facilities, and contaminated well GW01. 


\section{Background Information and Previous Studies}

The Hilton site is located in central McPherson County, approximately 4 mi north of the city of McPherson (Figure 1.1). The area surrounding Hilton is relatively flat and sparsely populated and is used mainly for agriculture. On the basis of 1985 mapping and U.S. Census data, the KDHE estimated the population within a 1-mi radius of the Hilton site at approximately 20. No formal community or political infrastructure is in place at the site. The grain storage facilities (the main structures at the site) have been operated by various entities.

\subsection{Background Information}

Carbon tetrachloride contamination in groundwater at Hilton was first identified in association with the 1992 sale of the private grain storage facility by Wall-Rogalsky to the current owner, Mid-Kansas Cooperative Association. Sampling of groundwater from a well (GW01) at a former grain storage facilities east of the railroad tracks indicated an elevated carbon tetrachloride concentration of $910 \mu \mathrm{g} / \mathrm{L}$. Groundwater contamination has continued to be found in well GW01 at this level or at higher levels. No contamination has been detected at any other groundwater sampling location, east or west of the former railroad tracks.

The contamination found in well GW01 (east of the former railroad tracks) has been linked to former grain storage activities at Hilton. The private grain storage facilities east of the former railroad tracks are the most likely source of the contamination because of their proximity to contaminated well GW01. Contaminants found in well GW01 include carbon tetrachloride (maximum of $1,630 \mu \mathrm{g} / \mathrm{L}$ in 1994), chloroform $(46.8 \mu \mathrm{g} / \mathrm{L})$, ethylene dibromide $(7.2 \mu \mathrm{g} / \mathrm{L})$, 1,2-dichloroethane $(1.9 \mu \mathrm{g} / \mathrm{L})$, 1,2-dichloropropane $(1.4 \mu \mathrm{g} / \mathrm{L})$, and tetrachloroethylene $(3.6 \mu \mathrm{g} / \mathrm{L})$.

In earlier investigations, carbon tetrachloride was detected in limited soil and vegetation sampling east of the former railroad tracks (Argonne 1997a,b). The CCC/USDA operated its grain storage facility west of the tracks in 1954-1965. 


\subsection{Previous Investigations}

In response to the discovery of contamination in well GW01, the KDHE sampled six domestic and irrigation wells (including two wells at the Ledell residence) within approximately $1.25 \mathrm{mi}$ northeast, west, and southwest of the former grain storage facilities. This sampling was part of a preliminary assessment in November 1992 (KDHE 1993). No carbon tetrachloride contamination was identified in groundwater in any of the sampled wells (Figure 2.1).

In August 1994, the KDHE returned to Hilton to collect a suite of shallow soil samples (at $8 \mathrm{ft}$ and $17 \mathrm{ft} \mathrm{BGL}$ ) within the former grain storage facilities along both sides of the railroad tracks. Carbon tetrachloride was found at trace concentrations $(0.13-0.26 \mu \mathrm{g} / \mathrm{kg})$ at three locations east of the tracks (KDHE 1994). A groundwater sample from GW01 contained carbon tetrachloride at $1,630 \mu \mathrm{g} / \mathrm{L}$ and chloroform at $46.8 \mu \mathrm{g} / \mathrm{L}$. Ethylene dibromide, 1,2-dichloroethane, 1,2-dichloropropane, and tetrachloroethylene were also detected in GW01. Complete analytical results for the Hilton site are in Appendix A, Table A.1. Locations of all previous sampling activities are shown in Figure 2.1.

At the request of the KDHE, Argonne conducted Phase I and Phase II investigations in 1996 and 1997 on behalf of the CCC/USDA. The purpose was to characterize the distribution of the carbon tetrachloride contamination, the stratigraphic units hosting contaminant migration, and local hydrogeology. The investigation involved five CPT pushes, three conventionally drilled soil borings (SB01, SB06, and SB07), a geophysical survey, and shallow soil and vegetation sampling. Three monitoring wells (SB01, SB06, and SB07) and two piezometers (SB04 and SB05) were installed (Argonne 1997a,b). Six private wells were sampled, including three of the wells sampled by the KDHE in 1992. Results of groundwater sampling from the Argonne-installed wells and piezometers, from GW01, and from domestic wells DW01-DW06 are shown in Figure 2.1. Detailed results are in Appendix A, Table A.1.

In August 2005, the KDHE sampled groundwater at seven residences near Hilton (Carey 2005). The results are displayed in Figure 2.1 and are included in Appendix A, Table A.1.

\subsubsection{Geologic and Hydrogeologic Setting}

The characteristics of the geologic and hydrogeologic setting at the Hilton site, as determined in Argonne's Phase I and Phase II investigations, are as follows (Argonne 1997a,b): 
- The shallow geologic section at Hilton (Figure 2.2) consists of Middle Pleistocene age fluvial and overbank sediments of the McPherson Formation, deposited along the eastern flank of the McPherson paleochannel, overlying a Middle Permian shale of the Wellington Formation. The overbank deposits are mainly calcareous silty clays and clays that overlie the sands of the fluvial facies, which is characterized as the part of the Equus Beds aquifer (a part of the regional High Plans aquifer; Figures 2.3-2.5). Protection of this aquifer is a motivating force for investigation of the contamination found in well GW01.

- The sands in the basal portion of the McPherson Formation that form the main Hilton aquifer are part of Equus Beds. This main Hilton aquifer unit (Equus Beds) is present at SB01 and SB07 (in the vicinity of the former private grain storage facilities east of the railroad) but is absent at SB06 (about 1,200 ft west of the railroad). At SB06 the main Hilton aquifer unit is represented by a series of gray to gray-brown, very dense clays. Wells SB01 and SB07 and piezometers SB04 and SB05 are screened in the main Hilton aquifer unit (Equus Beds). These relationships are illustrated in Figures 2.3-2.5.

- An upward-fining sequence of coarse-grained sand to fine- to very finegrained sand occurs in thin lenses, in a predominantly silty to sandy clay matrix. This sequence forms the shallow aquifer unit at the Hilton site. The sand unit is present in the upper portion of the McPherson Formation at SB06, but it pinches out eastward and southward and is absent at SB01 and SB07 (Figures 2.4 and 2.5). The well at location SB06 is screened in the shallow aquifer unit.

- The main Hilton aquifer unit (Equus Beds) may be locally confined or semiconfined in the vicinity of Hilton. The sands of this aquifer thin locally to slightly over $14 \mathrm{ft}$ in thickness. The wells completed at or near Hilton in the less developed, thinner sands of the Equus Beds aquifer (at the edge of the McPherson paleochannel) are reported to produce significantly less water (2-15 gpm) than wells to the west and southwest, developed in the thicker sections of the McPherson paleochannel, where the sequence is dominated by coarse sand and gravel. Irrigation wells developed in this coarser-grained section are reported to produce water at rates in excess of 1,000 gpm. 
- The mean potentiometric surface, based on January 1997 regional water level data (KGS 1997), declines from an elevation of $1,548 \mathrm{ft}$ above mean sea level (AMSL) at the eastern edge of McPherson County to 1,400 ft AMSL in the McPherson paleochannel at the western edge of the county. However, the January 1997 water levels in the vicinity of Hilton were between 1,438 ft and 1,440 $\mathrm{ft}$ AMSL, with a groundwater flow bearing of $\mathrm{S} 6^{\circ} \mathrm{W}$ (Figure 2.6). The average gradient in the observation well network in 1997 was 0.0004 , with a range of 0.0003-0.0005 at that time. (The gradient is expressed as a unitless fraction or decimal number.)

\subsubsection{Groundwater and Soil Contamination}

The characteristics of the groundwater and soil contamination at the Hilton site are as follows (Argonne 1997a,b):

- Carbon tetrachloride and chloroform have been found persistently in well GW01. However, no contamination was found in monitoring wells and piezometers or in domestic wells sampled in the vicinity of the Hilton site (Figure 2.1).

- The results of vegetation and soil sampling — outside the railroad property and surrounding private land to which access was denied - indicated the presence of low concentrations of carbon tetrachloride in a defined area on the eastern edge of the former private grain storage facilities east of the railroad tracks and south of Pathfinder Lane (Figure 2.7).

- The source of the contamination in well GW01 cannot be determined without access to the property on which the well is located. In the absence of additional evidence regarding the contaminant distribution at Hilton, the contaminant source can most reasonably be assumed to be near well GW01. The exact nature and length of the gravel pack surrounding the well is unknown. However, the possibility remains that well GW01 provided a conduit through which contaminants located higher in the saturated silts and clays or in the vadose zone reached the aquifer sands in the screened interval. 
In August 2005, the KDHE sampled groundwater at seven residences near Hilton, including four wells sampled in previous investigations (Carey 2005). The results confirmed the previously observed absence of carbon tetrachloride contamination in groundwater surrounding the former grain storage facilities at Hilton (Figure 2.1).

\subsection{Identification of the Former Grain Storage Facilities}

The results of Argonne's 2005 property records search are illustrated in Figure 2.8. The records found are in Appendix B. The results indicate that grain storage facilities formerly operated at Hilton in the following three areas:

- A western facility, west of the railroad tracks (location of the former $\mathrm{CCC} / \mathrm{USDA}$ operation)

- A northeastern facility, east of the railroad tracks and north of Pathfinder Lane (location of private operations on the railroad right-of-way)

- A southeastern facility, east of the railroad tracks and south of Pathfinder Lane (location of private operations on the railroad right-of-way and on adjacent privately owned property)

Historical aerial photos taken in 1950, 1956, 1963, and 1970 indicate that the grain storage facilities reached their maximum size during the 1960s (ASCS 1950, 1956, 1963, 1970). At the largest operational scale, the following structures are evident in the 1963 aerial photo (Figure 2.9):

- Western facility: 30 grain storage bins (20 ft in diameter) - operated by the CCC/USDA in 1954-1965

- Northeastern facility: 1 grain elevator, 1 storage shed and shop, 2 bins (20 ft in diameter), 9 silos, and contaminated well GW01 (south of the storage shed and shop) - operated by private entities 
- Southeastern facility: 1 elevator, 5 small bins ( $20 \mathrm{ft}$ in diameter), 6 silos, and 39 large bins ( $25 \mathrm{ft}$ in diameter) - operated by private entities

The preliminary assessment report (KDHE 1993) described former CCC/USDA grain storage operations on both sides of the tracks at Hilton. In January 1995, the KDHE issued a draft administrative order on consent (AOC) pertaining to Hilton site, identifying the CCC/USDA and Wall-Rogalsky Milling Company as respondents (KDHE 1995). The draft AOC indicated that the CCC/USDA had operated facilities at Hilton for an unidentified period prior to 1963. The draft AOC included the 1963 aerial photo (Figure 2.9), showing approximately 75 metal grain bins on both the west and east sides of the railroad tracks.

Argonne's 2005 records search, on behalf of the CCC/USDA, addressed Section 32, Township 18, Range 3 west, where the former Hilton grain storage facilities are located (Figure 2.9). The property documentation obtained from the McPherson County Register of Deeds office (Appendix B) includes all property transaction and ownership records related to the CCC/USDA operation, as well as additional documentation related to private grain storage operations at Hilton.

The 2005 review of property documents, in conjunction with analysis of aerial photos taken in 1950,1956, 1963, and 1970, led to the following conclusions (Figure 2.8):

- Conclusions with regard to the former CCC/USDA grain storage facility (western facility):

- The only property at Hilton historically leased to the CCC/USDA was on the west side of the railroad tracks. All of the lease documents identify the CCC/USDA leasehold as a strip of land $128 \mathrm{ft} \times 510 \mathrm{ft}$, west of the railroad right-of-way and north of Pathfinder Lane. This property was initially leased to the CCC/USDA by Keith Peterson in 1954 for five years. The lease was extended twice, for five years each time, for the periods 1959-1964 and 1964-1969. The second lease extension (19641969) was terminated early by a discharge of lease dated on April 30 , 1965 (Appendix B). The current deed (Appendix B) shows that since October 6, 2003, the property has been owned by Foster Farms, a Kansas partnership. 
- The historical aerial photographic record of grain storage activity on the Hilton western facility is consistent with the lease record, which places the CCC/USDA operation in the period 1954-1965. A 1950 aerial photo shows no bins on the former CCC/USDA facility. Consistently from 1956 through 1963, 30 metal bins (each $20 \mathrm{ft}$ in diameter) are visible on the CCC/USDA facility. By 1970, all traces of structures on the former CCC/USDA facility had been removed.

- Conclusions with regard to private grain storage facilities on the former Union Pacific Railroad right-of-way (northeastern facility and part of the southeastern facility):

- The former railroad right-of-way in the vicinity of Hilton was owned by the Union Pacific Railroad Company from the end of the 19th century to 1997.

- In 1997, the former Union Pacific Railroad right-of-way was conveyed to the Central Kansas Conservancy (Appendix B), along with the entire 33.4-mi rail line in McPherson and Marion Counties (American Trails 2003). Included was the section of the former railroad between Linsborg and McPherson, which runs through the Hilton site. The conveyance was for "rail banking/interim trail use." The land is now used as a walking and biking trail.

- The 1997 conveyance of the railroad track and right-of-way to the Central Kansas Conservancy specifically excluded three tracts previously or currently occupied by part of the southeastern private grain storage facility. These three 100-ft-wide tracts lay on the former railroad right-ofway south of Pathfinder Lane (Appendix B, Table B.1). A fourth 100-ftwide tract currently occupied by the northeastern private grain storage facility was not specified in the 1997 transaction. Both the northeastern and southeastern facilities are currently operated by the Mid-Kansas Co-op (Figure 2.8). No public documentation has been found regarding the current ownership of these properties. The ownership of an adjacent $33-\mathrm{ft}$ by $660-\mathrm{ft}$ tract is discussed below. 
- The former private grain storage facilities on the east side of the railroad tracks lay mainly on the former railroad right-of-way. The 1950 and 1956 aerial photos show only a few silos and elevators on the east side of the tracks. In the 1963 photo (Figure 2.9), 46 grain storage bins are visible on the east side of tracks, 28 within the railroad right-of-way. By 1970, most of these bins had been removed.

- Railroad companies had no legal obligation to file leases with county governments. No leases for the grain storage facilities on the former Union Pacific Railroad right-of-way at Hilton were filed with the county prior to 1997 (when Argonne was completing its Phase II investigation). Nevertheless, the private operator of the grain storage facility on railroad property at Hilton can be inferred from the historically documented ownership of the facility's extension eastward from the railroad right-ofway (see below).

- Conclusions with regard to the southeastern private grain storage facility outside the railroad right-of-way:

- The southeastern private grain storage facility expanded eastward from the railroad right-of-way to a strip of land, $33 \mathrm{ft}$ by $660 \mathrm{ft}$, originally owned by Lila and Elmer Tector (Figure 2.8). The McPherson Grain Company purchased this strip of the land from the Tectors in 1959 and sold it to Wall-Rogalsky Milling Company in 1981. In 1992, the land was sold to the current owner, Mid-Kansas Cooperative Association (Appendix B). The Mid-Kansas Co-op currently operates facilities both on this strip of land and on properties within the former railroad right-of-way, as indicated above.

- An expanded southeastern private grain storage facility on the strip of land next to the railroad right-of-way, including a row of 18 grain storage bins (each $25 \mathrm{ft}$ in diameter), is first visible in the 1963 photo (Figure 2.9). This observation is consistent with the property transfer from the Tectors to the McPherson Grain Company in 1959. Though no conclusive evidence exists, a reasonable assumption is that the same company operated 
facilities on the leased right-of-way and expanded eastward in or before 1963 to increase its storage space.

\subsection{Access Issues}

During Argonne's Phase I and Phase II investigations in 1996 and 1997, the CCC/USDA was unable to gain access to the railroad right-of-way and to adjacent privately owned lands. Argonne's investigations were therefore limited to the narrow strip of land owned by MidKansas Co-op (Figure 2.8) and to the shoulders of township and county roads.

In a July 1998 letter, the USDA requested assistance from U.S. Environmental Protection Agency (EPA) Region VII in obtaining access to the railroad right-of-way (Gurwitz 1998). The EPA regional counsel responded that because no further response actions were planned at Hilton under Superfund and the KDHE was assuming leadership for the site, access issues would become the concern of the KDHE (Kahn 1998).

In view of the findings of the 2005 property records search, the access permits required in the targeted investigation proposed here will initially be limited to Foster Farms, the Ledell property west of the former railroad, and the Central Kansas Conservancy (Figure 2.8). 


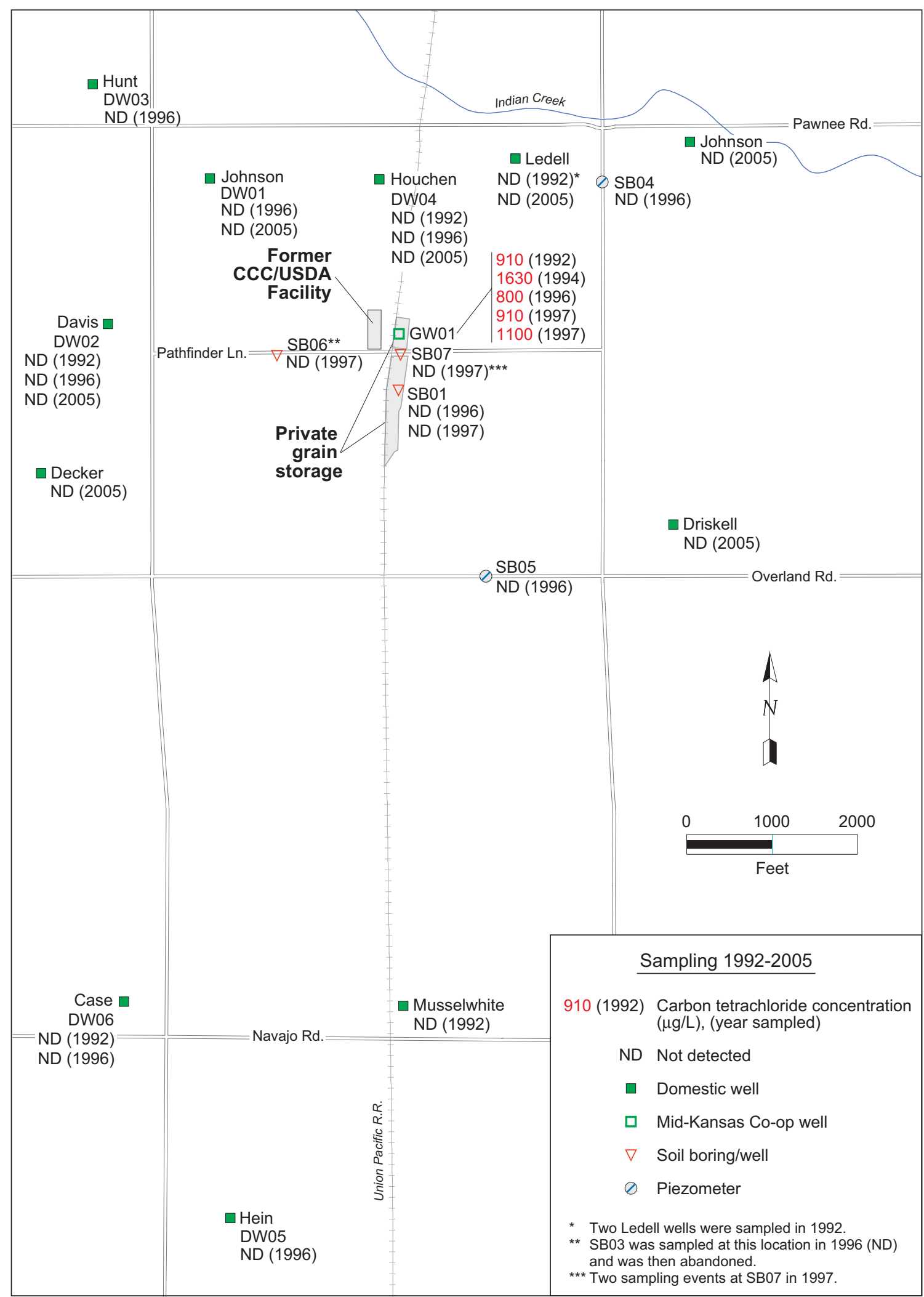

FIGURE 2.1 Historical results of carbon tetrachloride analyses on groundwater samples collected in the Hilton area. 


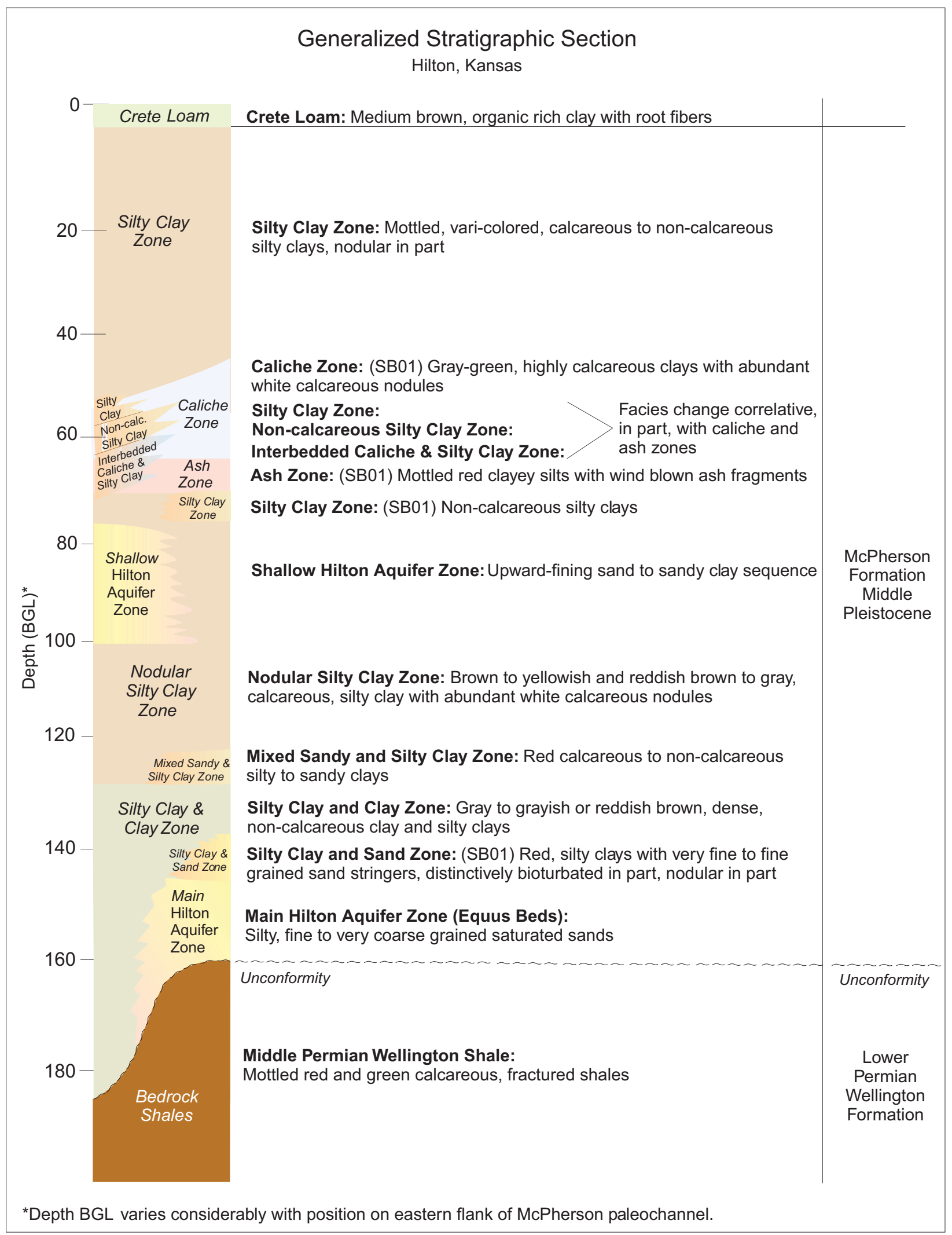

FIGURE 2.2 Simplified stratigraphic section derived from core descriptions for SB01, SB06, and SB07 at Hilton. 


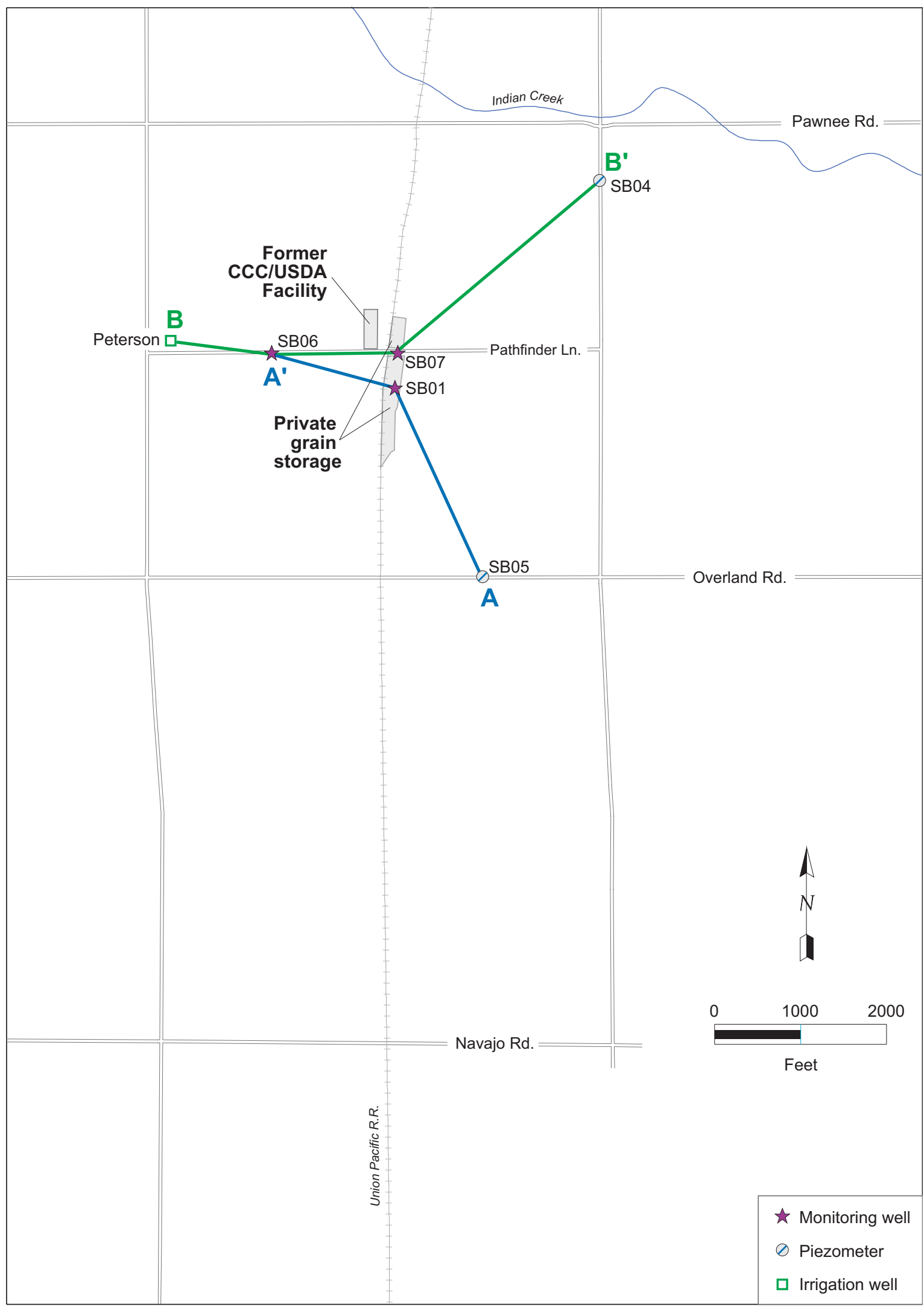

FIGURE 2.3 Locations of monitoring wells, piezometers, and geologic cross sections. 


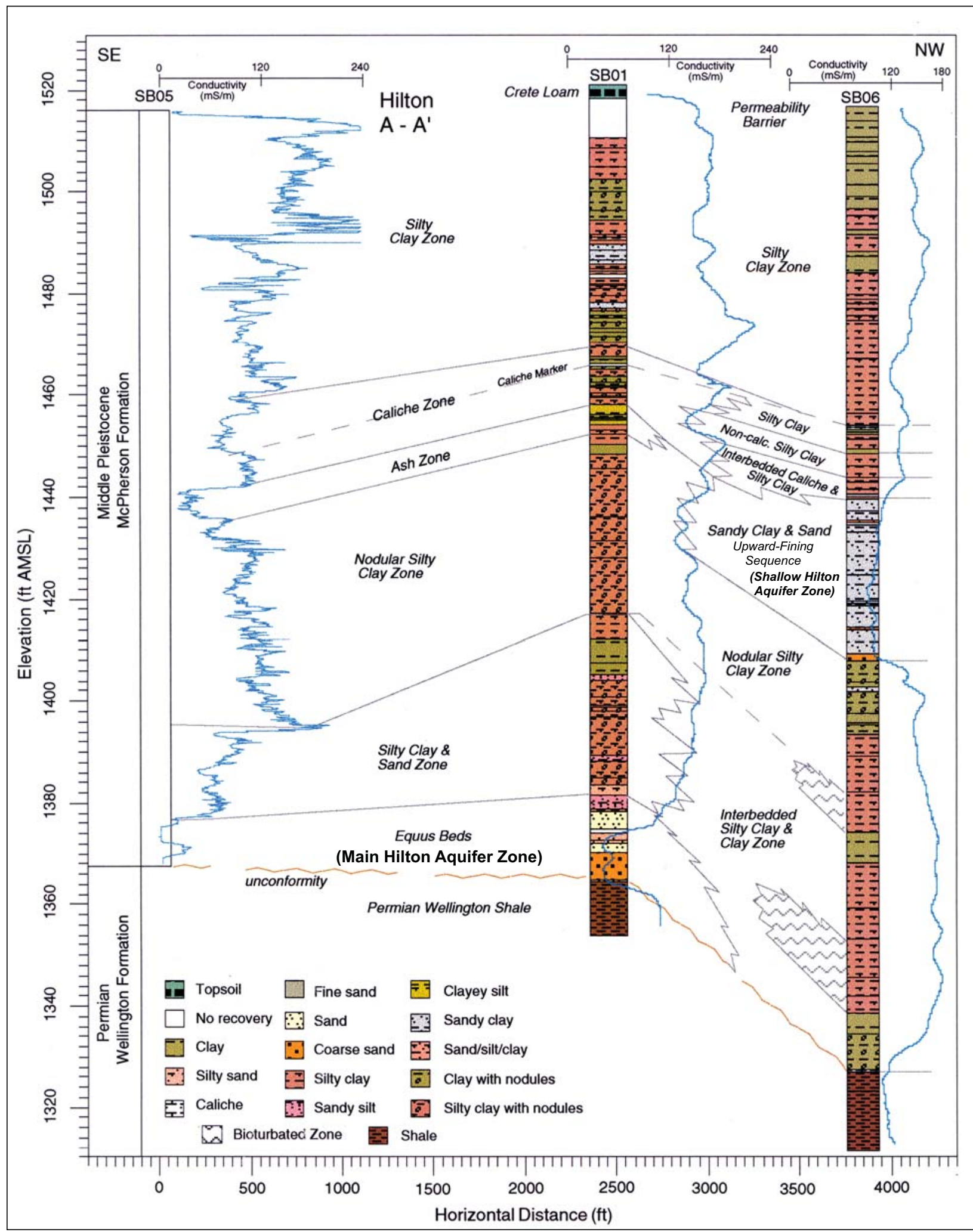

FIGURE 2.4 Southeast-to-northwest geologic cross section A-A' (vertically exaggerated), illustrating the stratigraphic relationships in the vicinity of the former CCC/USDA and private grain storage facilities at Hilton. 


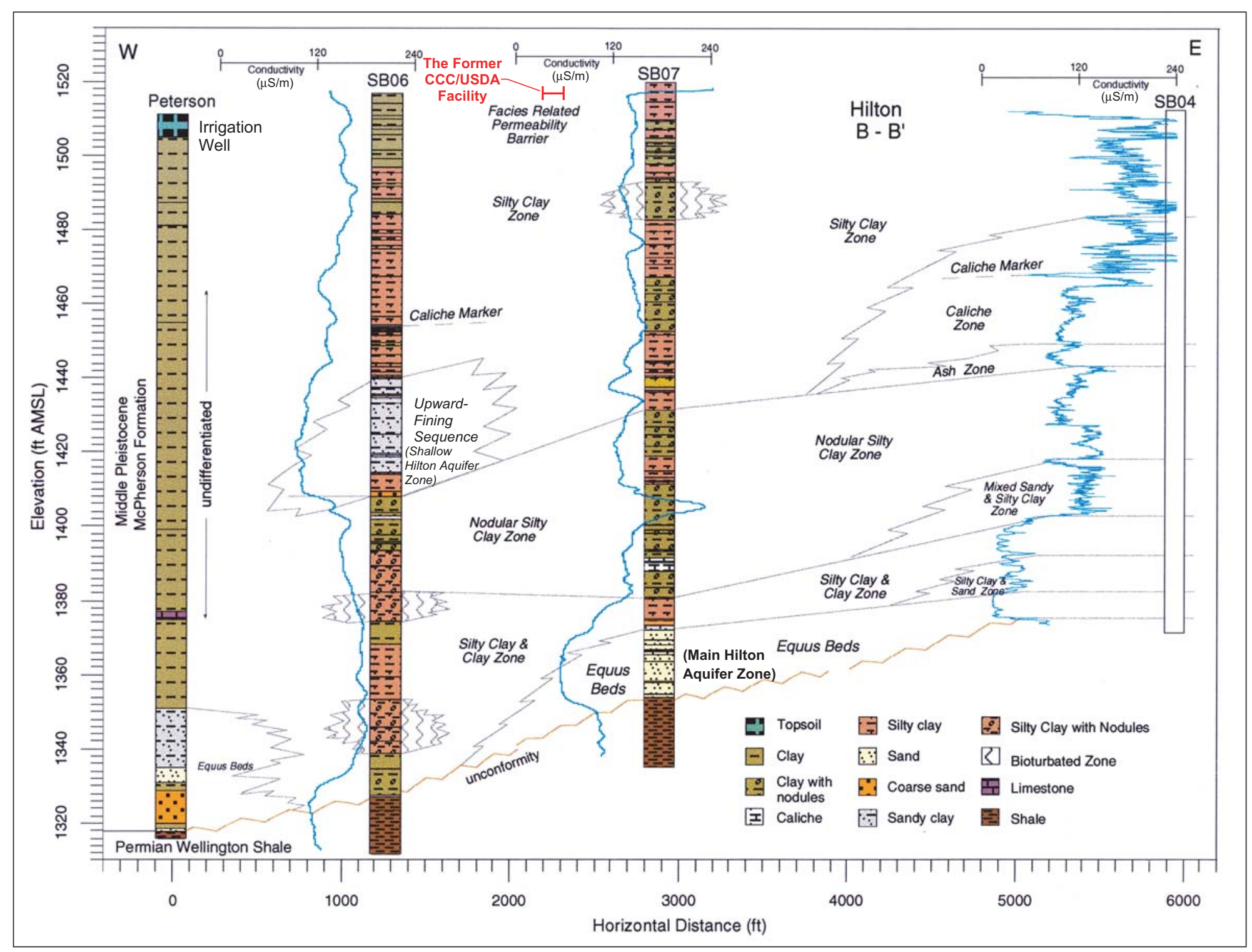

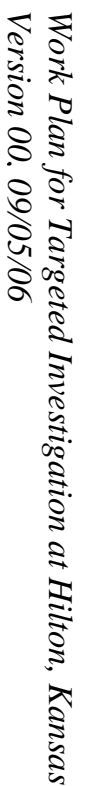

FIGURE 2.5 West-to-east geologic cross section B-B' (vertically exaggerated), illustrating the stratigraphic relationships in the vicinity of the former CCC/USDA and private grain storage facilities at Hilton. 


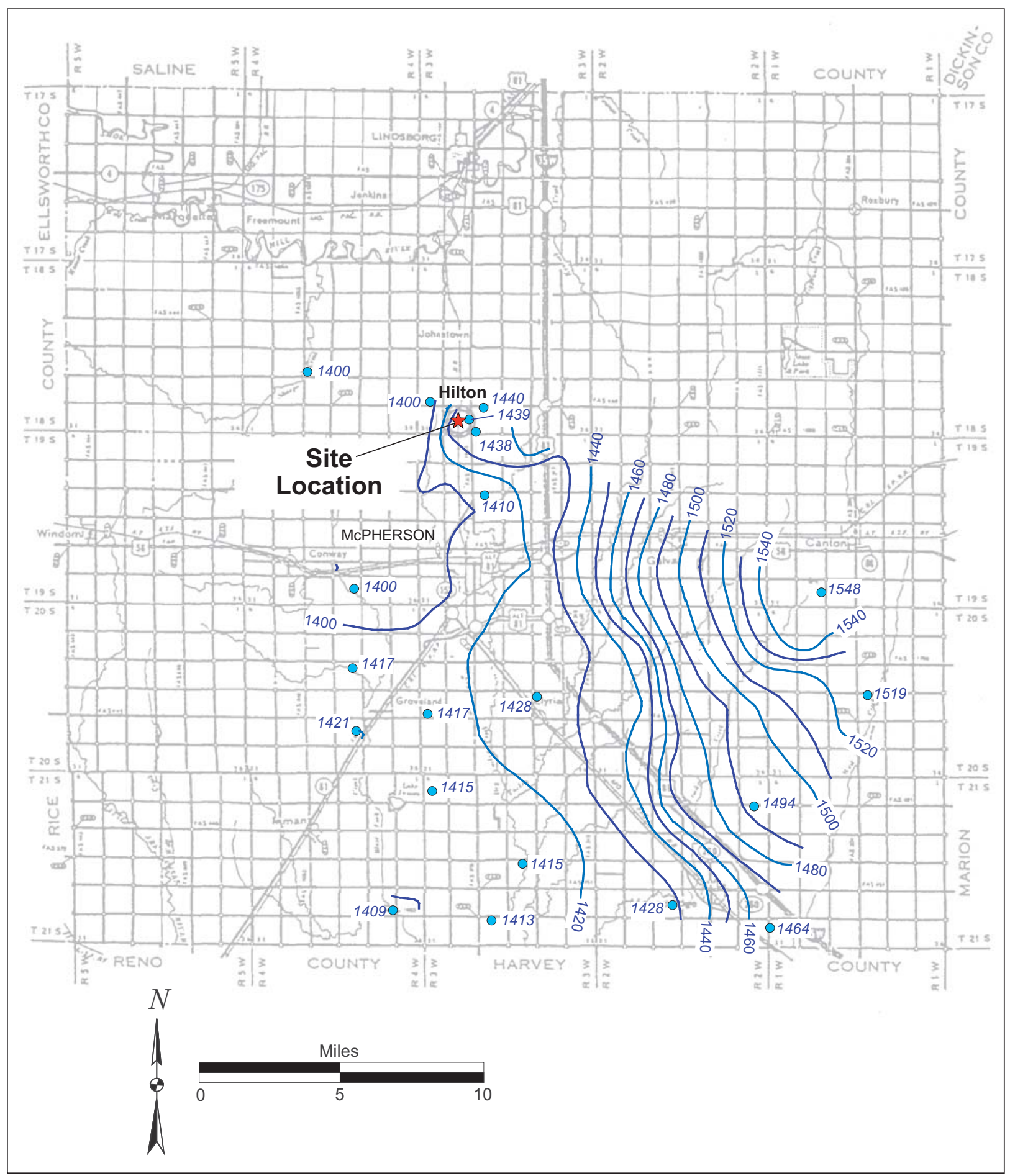

FIGURE 2.6 Regional potentiometric surface (ft AMSL) for the Equus Beds aquifer, McPherson County, Kansas, prepared from Kansas Geological Survey records (KGS 1997) and Argonne Phase II water level data. 


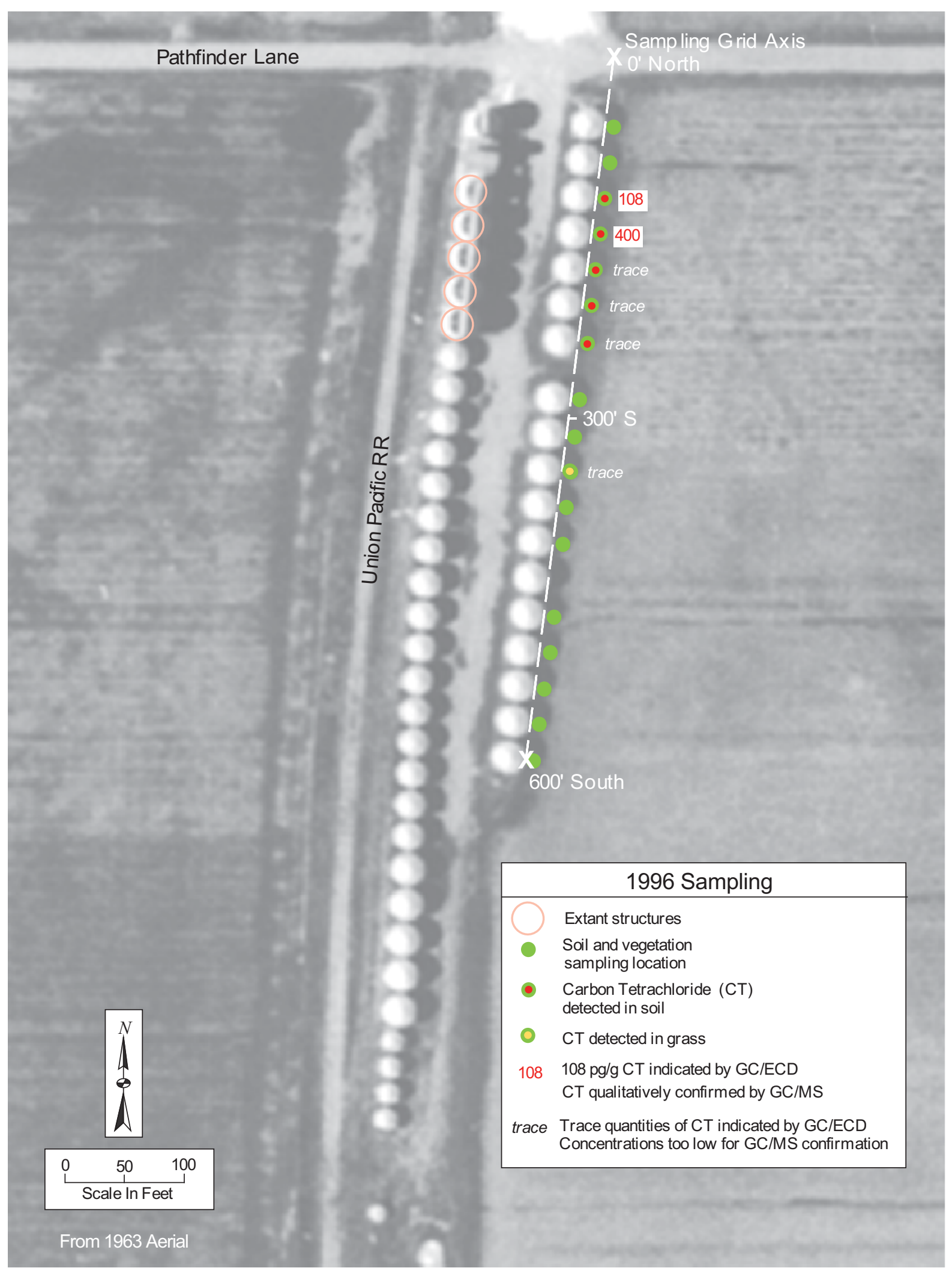

FIGURE 2.7 Soil and vegetation sampling locations (1996) and analytical results for carbon tetrachloride, shown on a 1963 aerial photograph. Source of photograph: ASCS (1963). 


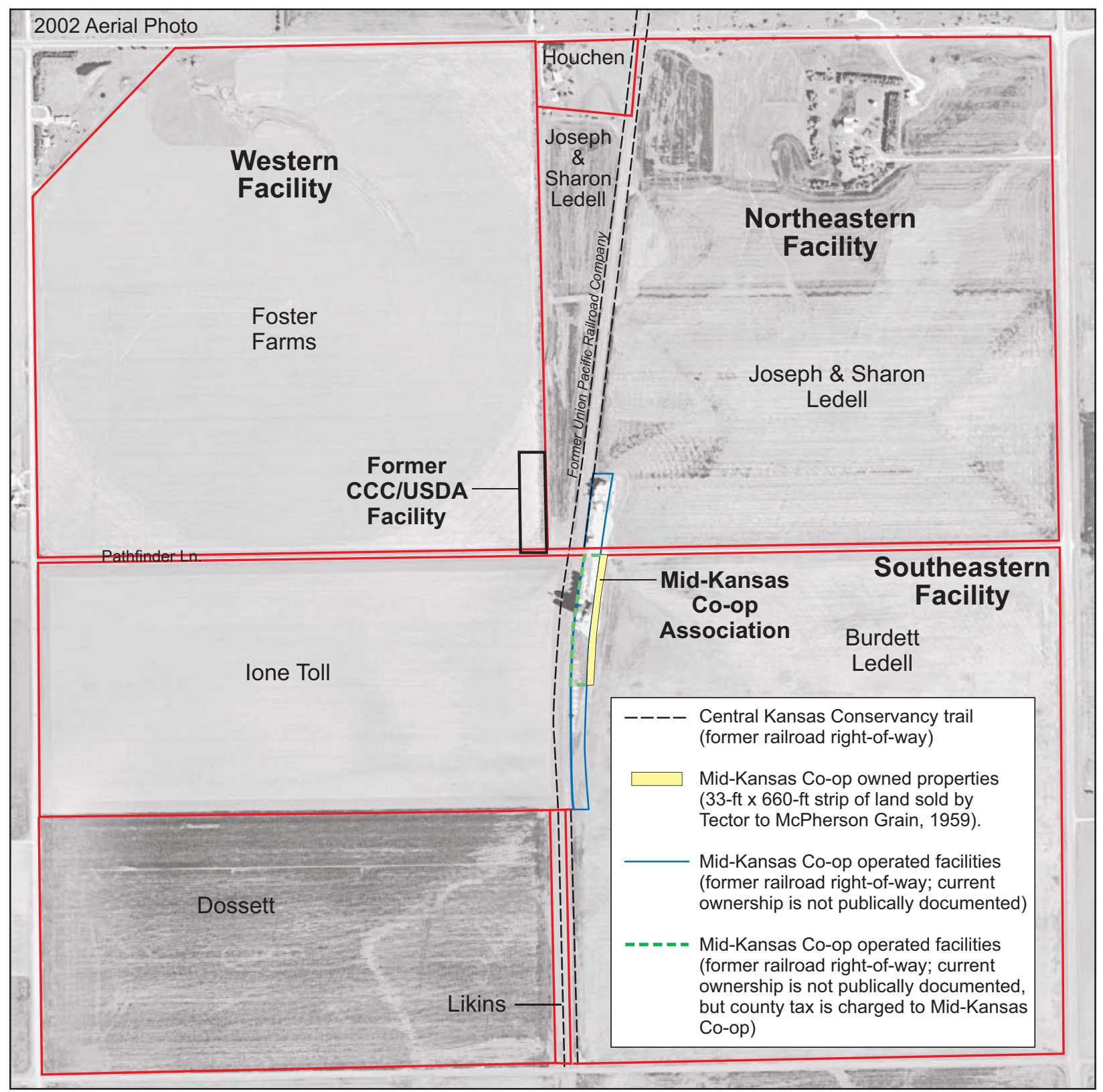

FIGURE 2.8 Locations of current property boundaries, the former CCC/USDA facility, the present MidKansas Co-op, and the former Union Pacific Railroad Company property at Hilton. The former railroad property is now owned in part by the Central Kansas Conservancy and occupied in part by the MidKansas Co-op facilities. Source of photograph: NAIP (2002). 


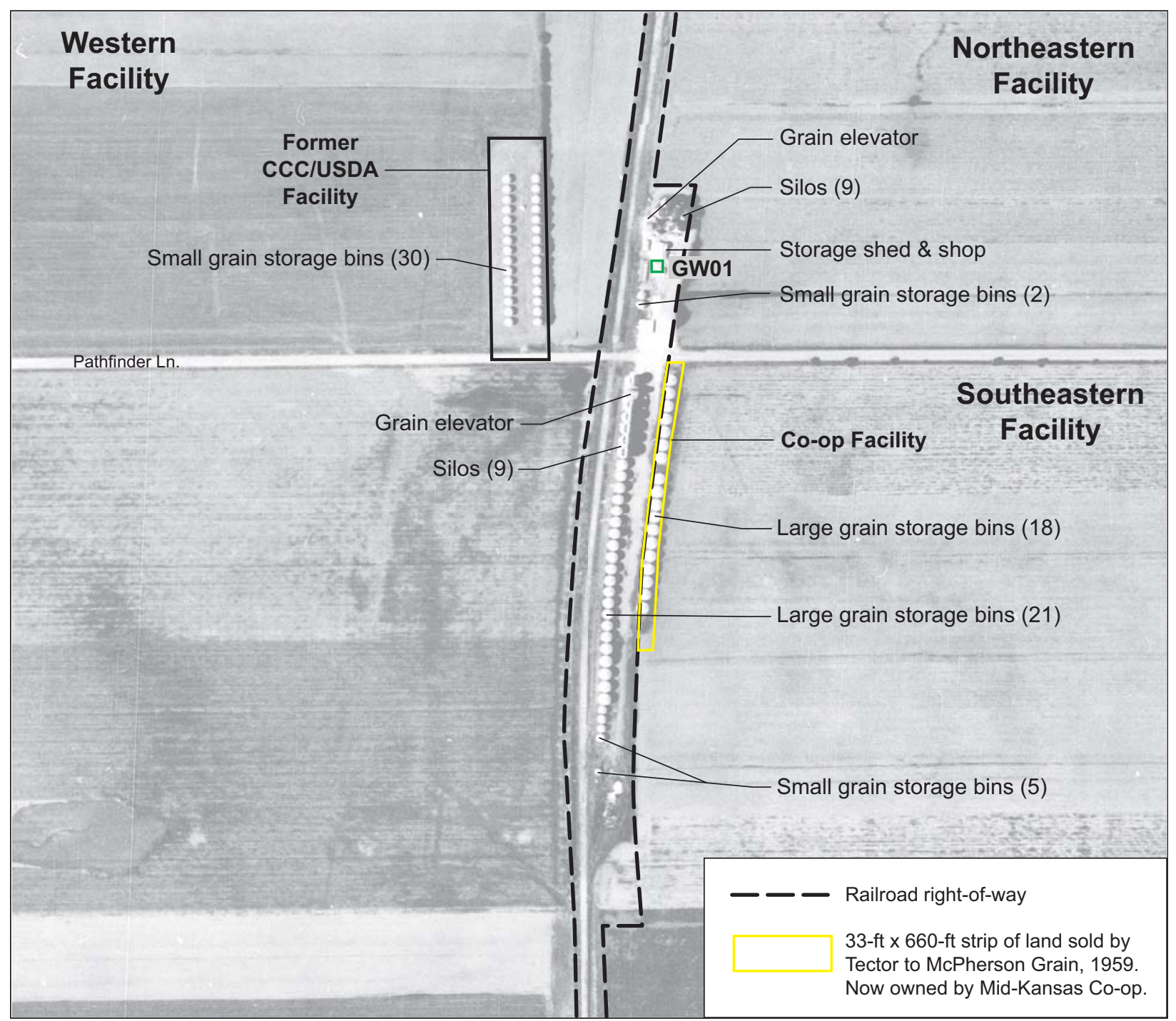

FIGURE 2.9 The maximum scale of grain storage facilities at Kansas, interpreted from a 1963 aerial photograph. Source of photograph: ASCS (1963). 


\section{Technical Program}

The investigative program outlined here focuses on potential groundwater contamination related to the former CCC/USDA facility west of the former railroad tracks. A phased approach is proposed, with four segments of investigation to determine whether carbon tetrachloride is present in soil and groundwater at the former CCC/USDA facility, then define the extent of the potential contamination.

\subsection{Project Objectives}

The technical objectives of the proposed work are as follows:

- Investigate for carbon tetrachloride contamination in shallower soil and the shallow aquifer unit on the former CCC/USDA property (west of the former railroad) to determine whether a soil source of carbon tetrachloride and a soilto-groundwater migration pathway exist on the property.

- If contamination is found in shallower soil and/or the shallow aquifer unit on the former CCC/USDA property, extend the investigation for carbon tetrachloride contamination downward to the main aquifer unit, and determine the groundwater flow direction.

- If a significant contaminant source is found on the former CCC/USDA property, determine the lateral and vertical extent of the carbon tetrachloride plume emanating from the property.

- If the former CCC/USDA facility is identified as a contaminant source, establish a groundwater monitoring network.

\subsection{Scope of Work}

The proposed work will be accomplished in four segments addressing the four technical objectives in Section 3.1. The results from each segment will be evaluated in the field to 
determine whether the activities proposed for the next segment are appropriate and necessary. The CCC/USDA and KDHE project managers will be informed of the results of each segment of the investigation and will jointly determine whether the work is to progress to the next segment.

As new data are acquired during the field program, the recommended tasks for each segment may be revised to reflect an improved understanding of the site. Modifications could include the reduction or expansion of certain activities, as well as the elimination of elements of the program judged to be unnecessary to meet the stated technical objectives. Any changes to the Work Plan will be made in consultation with the CCC/USDA and KDHE project managers.

Detailed procedures for the individual activities recommended by Argonne are presented in the Master Work Plan (Argonne 2002) for environmental investigations in Kansas.

\subsubsection{Segment 1: Investigate for Carbon Tetrachloride Contamination in Shallower Soil and the Shallow Aquifer Unit on the Former CCC/USDA Property}

Investigation Segment 1 will address the former CCC/USDA property west of the former railroad. The planned activity in Segment 1 is as follows:

- Activity 1: At five proposed locations on the former CCC/USDA property, use the cone penetrometer (CPT) unit to collect subsurface soil samples in vertical profiles at intervals of $4 \mathrm{ft}$ or at changes in lithology, to the depth of refusal. If the shallow aquifer unit is encountered (at a depth of approximately $80 \mathrm{ft}$ BGL), collect groundwater samples, install temporary piezometers, and determine the groundwater flow direction. Analyze the soil samples for volatile organic compounds (VOCs); analyze the groundwater samples for VOCs and 1,2-dibromoethane.

Of the five proposed CPT investigation locations, the initial three are shown in Figure 3.1. The remaining CPT locations will be selected in consultation with the CCC/USDA and KDHE project managers as the investigation proceeds, to ensure optimal placement on the basis of the initial results. 
At each of the five proposed locations, the CPT unit will be used to collect soil samples in vertical profiles at intervals of $4 \mathrm{ft}$ or at changes in lithology, to the depth of refusal or to the shallow aquifer. All soil samples will be analyzed for VOCs to identify the presence and concentration of carbon tetrachloride and chloroform.

The hydrogeologic setting beneath the former CCC/USDA facility is expected to be similar to the section illustrated in Figure 3.2, interpreted from Argonne's Phase I and Phase II investigations (Argonne 1997a,b). Verticalprofile soil sampling will continue, if penetration is achieved, to the shallow aquifer unit. This unit is composed of an upward-fining sequence from coarsegrained sand to a fine- to very fine-grained sand occurring in thin lenses in a silty to sandy clay matrix at $78-108 \mathrm{ft}$ BGL. The shallow aquifer unit is locally distributed and in previous investigations has been encountered only at SB06, 1,000 ft west of the former CCC/USDA facility. The shallow aquifer unit will be most vulnerable to contamination if it is present beneath the former CCC/USDA facility.

Groundwater samples for analyses for VOCs and 1,2-dibromoethane will be collected within the shallow aquifer unit below the former CCC facility. Analytical results for these groundwater samples will indicate whether groundwater directly under the former CCC/USDA facility is contaminated with VOCs potentially related to overlying sources.

Temporary 1-in.-diameter observation piezometers will be installed with the CPT at each of the investigated locations where the upper aquifer unit is encountered. Groundwater levels will be measured in these piezometers to determine the flow direction beneath the former CCC/USDA facility. Any temporary observation points will be abandoned in accordance with KDHE requirements upon completion of the field investigation. Alternately, the temporary observation points can later be converted to permanent installations at the discretion of the CCC/USDA and KDHE project managers, if access is granted (Segment 4). 
Segment 1 of the investigation will end upon completion of the activities described above. The presence of contamination in shallower soil and/or the shallow aquifer unit beneath the former CCC/USDA facility will be evaluated as the main criterion for determining whether the activities in Segment 2 of the investigation are appropriate and necessary.

\subsubsection{Segment 2: If Contamination Is Found in Shallower Soil and/or the Shallow Aquifer Unit on the Former CCC/USDA Property, Extend the Investigation for Carbon Tetrachloride Contamination Downward to the Main Aquifer Unit, and Determine the Groundwater Flow Direction}

Investigation Segment 2, if warranted, will further address the former CCC/USDA property west of the former railroad. The planned activities in Segment 2 are as follows:

- Activity 2a: To determine whether the former CCC/USDA facility contributed to the contamination identified in the lower aquifer at well GW01, use the CPT (or a sonic drilling rig if necessary) to collect soil samples in vertical profiles at the locations investigated in Segment 1 at which carbon tetrachloride was found. When the main aquifer is encountered (at a depth of approximately $170 \mathrm{ft} \mathrm{BGL}$ ), collect groundwater samples. Analyze the soil samples for VOCs; analyze the groundwater samples for VOCs and 1,2-dibromoethane.

In this activity, CPT pushes, or drilled boreholes as necessary, will be advanced through the entire McPherson Formation to the underlying red and green calcareous, fractured shales, at an approximate depth of $170 \mathrm{ft}$ BGL. The main Hilton aquifer unit (Equus Beds), which consists of silty, fine to very coarse-grained sands, is expected at the basal portion of the McPherson Formation (about 150-170 ft BGL), with a thickness of less than $15 \mathrm{ft}$.

- Activity 2b: For groundwater level measurements, install temporary piezometers (if CPT installations are possible) or permanent piezometers (if drilling is required) in the main aquifer at appropriate locations on the former CCC/USDA property investigated in Activity 2a. Measure groundwater levels in the available wells and piezometers. 
To determine the current groundwater flow direction, water level measurements will be made in the newly installed piezometers, in existing monitoring wells and piezometers SB01 and SB04-SB07, and in well GW01 (Figure 3.3). Information on the construction of existing wells and piezometers is in Table 3.1. Confirmation of the existence and condition of these wells and piezometers will be part of the field investigation.

- Activity 2c: Acquire detailed information, both in the field and from available records, regarding current and historical water usage and pumping rates from irrigation wells in the immediate vicinity of the Hilton site, to evaluate their potential to significantly affect the local hydrology.

The results of the vertical-profile sampling in Segments 1 and 2 will indicate whether a soil source of carbon tetrachloride and a soil-to groundwater migration pathway exist at the former CCC/USDA facility. Segment 3 of the investigation will be initiated if a significant contaminant source is confirmed at the former CCC/USDA facility.

TABLE 3.1 Construction information for existing wells and piezometers at Hilton, Kansas. ${ }^{a}$

\begin{tabular}{ccccc}
\hline Well/Piezometer & $\begin{array}{c}\text { Depth } \\
\text { (ft BGL) }\end{array}$ & $\begin{array}{c}\text { Diameter } \\
\text { (in.) }\end{array}$ & $\begin{array}{c}\text { Screen } \\
\text { Interval (ft BGL) }\end{array}$ & $\begin{array}{c}\text { Reference } \\
\text { Elevation } \\
\text { (ft AMSL) }\end{array}$ \\
\hline SB01 & 171.5 & 4 & $144-164$ & 1520.90 \\
SB04 & 135.7 & 1 & $130-135$ & 1512.36 \\
SB05 & 148 & 1 & $138-148$ & 1516.12 \\
SB06 & 205 & 4 & $99-109$ & 1516.12 \\
SB07 & 184 & 4 & $146.5-166.5$ & 1518.80 \\
GW01 & $166^{c}$ & Unknown & Unknown & 1520.16 \\
\hline
\end{tabular}

a The present condition of the SB wells and piezometers will need to be evaluated in the field before sampling.

b Elevation at the top of the well or piezometer casing.

c Depth reported by the KDHE (1993). 


\subsubsection{Segment 3: If a Significant Contaminant Source Is Confirmed on the Former CCC/USDA Property, Determine the Lateral and Vertical Extent of the Carbon Tetrachloride Plume Emanating from the Property}

Investigation Segment 3, if warranted, will address the wider area at Hilton. The planned activities in Segment 3 are as follows:

- Activity 3a: Conduct groundwater sampling for analyses for VOCs and 1,2-dibromoethane in existing monitoring wells and piezometers determined to be downgradient, as well as in selected upgradient well(s). Figure 3.3 shows the current existing monitoring wells and piezometers and also well GW01.

- Activity 3b: Conduct additional groundwater sampling for VOCs and 1,2-dibromoethane analyses at selected new upgradient and downgradient locations, through use of the CPT if possible.

Groundwater sampling at the locations hydraulically upgradient from the former CCC/USDA facility will help to define the sources contributing to groundwater contamination under the former CCC/USDA facility (if such contamination is found). The number and locations of sampling points will be determined in consultation with the CCC/USDA and KDHE project managers.

To delineate the groundwater plume originating from the former CCC/USDA facility, vertical-profile groundwater sampling through the aquifer units will be conducted at additional locations downgradient from the former CCC/USDA facility, along potential plume migration pathways. The additional number and locations of sampling points will be determined, in consultation with the CCC/USDA and KDHE project managers, on the basis of the local groundwater flow pattern and the contaminant distribution.

At each additional groundwater sampling location, electronic CPT logs will be acquired to identify the aquifer units, as described in Section 3.2.1, if penetration is achieved by the CPT unit. 
The results of activities in Segment 3 will permit the mapping of carbon tetrachloride concentrations in groundwater throughout the investigation area. The CCC/USDA and KDHE project managers will determine jointly whether the activities proposed for the next segment are appropriate and necessary.

\subsubsection{Segment 4: If the Former CCC/USDA Facility Is Identified as a Contaminant Source, Establish a Groundwater Monitoring Network}

Investigation Segment 4, if warranted, will address the wider area at Hilton. The planned activity in Segment 4 is as follows:

- Activity 4: Install permanent piezometers or monitoring wells at selected locations, both upgradient and downgradient from the former CCC/USDA facility, to form a network for monitoring of contaminant migration and seasonal groundwater level fluctuations, as well as for determination of the potentially significant effects of pumping from nearby irrigation wells on the groundwater flow pattern and contaminant migration pathways.

Locations for the permanent monitoring points will be selected in consultation with the CCC/USDA and KDHE project managers. Locations available will depend on the success of access negotiations.

Analyses of data generated in the investigation will provide the basis for determining what further $\mathrm{CCC} / \mathrm{USDA}$ activities are necessary at the Hilton site.

\subsection{Investigation Methods}

\subsubsection{Methods for Sampling of Existing Wells}

Water level measurement and sampling in existing wells will be conducted in accordance with the procedures described in the Master Work Plan (Argonne 2002), as follows: 
1. The well number, the well owner's name, or both will be documented in the site field notebook.

2. If possible, the static groundwater level and then the total depth will be measured and documented for each well.

3. The groundwater from each well will be purged until field parameters of $\mathrm{pH}$, temperature, and conductivity are stable. If possible, a minimum of three well volumes of water will be purged. The field parameters and volume purged will be documented. Each well will be purged before it is sampled.

4. The wells will be sampled after adequate recharge has occurred but no more than $24 \mathrm{~h}$ after purging.

5. Groundwater samples for analyses for VOCs (including carbon tetrachloride and chloroform) and 1,2-dibromoethane will be collected in laboratoryapproved containers and immediately placed in a cooler at $4^{\circ} \mathrm{C}$. These samples will be shipped for overnight delivery to the Applied Geosciences and Environmental Management (AGEM) Laboratory at Argonne for off-site analysis.

6. Any unavoidable deviations from these procedures will be documented in the field notebook.

\subsubsection{Methods for Groundwater Sampling with the Cone Penetrometer}

The groundwater samples collected with the CPT will be submitted to the AGEM Laboratory for rapid-turnaround (24-h) analyses, to facilitate review of the investigation results by Argonne and by the CCC/USDA and KDHE project managers during the field program.

At each location investigated with the CPT, logs of tip pressure, sleeve friction, and conductance will first be acquired to determine the basic lithostratigraphy and hydrostratigraphy of the site in the context of the local hydrogeologic setting. On the basis of these logs, selected 
depth intervals (to be determined in the field) may be chosen for coring, to provide sediment samples for lithologic confirmation and correlation of the CPT log responses.

At each location investigated with the CPT, depth intervals will be chosen in the field for groundwater sampling on the basis of the site-specific lithologic and hydrostratigraphic information outlined above. The groundwater sampling will be performed in accordance with the procedures described in the Master Work Plan (Argonne 2002), by first using the CPT rods to push a sacrificial tip and 0.5-in.-I.D. polyvinyl chloride (PVC) filter screen and riser to the desired maximum sampling depth. The rods will then be partially withdrawn to the desired minimum sampling depth, to expose the screen to the formation waters. Samples will be collected from the PVC casing by using a bailer, without purging, for preservation and analysis as described in Section 3.3.1 and Section 3.5.

The screen and riser used for sampling at selected locations will be left in place temporarily, to permit the periodic measurement of static groundwater levels during the field program. These temporary observation points will be abandoned in accordance with KDHE requirements upon completion of the field investigation. At the discretion of the CCC/USDA and KDHE project managers - and if access is granted — permanent piezometers or monitoring wells can be installed at these locations in accordance with procedures described in the Master Work Plan (Argonne 2002).

\subsubsection{Methods for Vertical-Profile Soil Sampling with the Cone Penetrometer}

When possible, soil sampling will be performed by using the CPT to obtain core samples from $10 \mathrm{ft}$ below ground surface to the top of the saturated zone. Soil samples will be taken at intervals of $10 \mathrm{ft}$ or less and/or at changes in lithology. The soil samples recovered will be placed in jars, sealed, preserved on dry ice in the field, and shipped to the AGEM Laboratory for preparation and analysis, in accordance with the Master Work Plan (Argonne 2002). A corresponding groundwater sample will be collected from the saturated zone directly beneath each soil sampling location, by using the methods described in Section 3.3.2, to permit direct evaluation of any potential soil source influence. 


\subsubsection{Methods for Soil and Groundwater Sampling with the Sonic Drilling Rig}

Groundwater samples collected with the drilling rig will be submitted to the AGEM Laboratory for rapid-turnaround (24-h) analyses. The rapid analyses will facilitate review of the results by Argonne and by the CCC/USDA and KDHE project managers during the field program and will support decision making that will drive the direction of the investigation.

At each location investigated, an inner drill string with core barrel will be advanced without drilling fluid - $10 \mathrm{ft}$ at a time. Next, an outer sonic casing will be advanced. Some potable water will be used for lubrication; the amount of water added for each interval will be monitored. The inner barrel will then be pulled, and the soil core will be extracted for lithologic confirmation. After this, the stainless steel screen will be installed and set at the bottom of the borehole with the lead rod and a K-packer or other device that forms a seal between the screen and casing. The outer casing will then be vibrated back $5 \mathrm{ft}$ or another desired interval, exposing the screen to the formation. An inflatable packer will be installed to isolate the screened zone. After purging of the volume of water added during advancement of the outer casing, plus five times the volume of the isolated sampling zone, the groundwater sample will be collected by using a bailer. Samples will be preserved and analyzed as described in Section 3.3.1 and Section 3.3.6.

\subsubsection{Methods for Installing Permanent Piezometers and Monitoring Wells}

Permanent monitoring points will be installed in accordance with Kansas regulations. Installation of piezometers (well points) with the CPT unit will follow the procedures in the Master Work Plan (Argonne 2002, Section 6.4.6 and Appendix F). A sand filter pack will be placed around the screened interval, and the remainder of the annulus will be sealed with bentonite grout.

If the subsurface lithologic conditions inhibit penetration with the CPT unit, a drilling rig will be used. Monitoring wells will be installed according to the general procedures in Section 6.4.3 of the Master Work Plan (Argonne 2002). The boreholes will be drilled by using a hollow-stem auger rig. The wells will consist of 2-in. PVC casing installed in 8.25-in.-diameter boreholes. Screens will be 0.010-in. mill slot, PVC, $10 \mathrm{ft}$ long. A 10/20 (or \#20) filter pack is anticipated, on the basis of previous experience in the area. The filter pack will extend $5 \mathrm{ft}$ below 
and $2 \mathrm{ft}$ above the screened interval. At the bottom of each well, a 5-ft section of blank casing capped on the bottom will serve as a silt trap.

All wells will be constructed in accordance with KDHE guidelines. Any variances required will be obtained from the appropriate agency prior to installation. All soil waste will be scarified on location. Development water will be discharged appropriately, as indicated by the results of confirmatory sampling and analysis for contaminants. Surface completions will consist of KDHE-approved flush mounts, as shown in the specifications for 2-in. casing in Figure F.4, Appendix F, of the Master Work Plan (Argonne 2002). After installation, each well will be pumped and developed for a minimum of $2 \mathrm{~h}$.

\subsubsection{Methods for Handling and Disposal of Investigation-Derived Waste}

The approach to handling and disposal of soil and water investigation-derived waste (IDW) is discussed in detail in Appendix C. Briefly, the approach is as follows:

- Soil cores collected during sampling will be retained in core boxes for lithologic description and research. The cores will be transported to and stored at an Argonne facility for further reference.

- Soil IDW from drilling activities will be stored on-site in 55-gal drums or a roll-off container. A representative sample will be collected and analyzed by a KDHE-certified laboratory.

- A Solid Waste Disposal Authorization will be obtained from the KDHE for disposal of soil in a permitted landfill. If analytical data indicate that the soils cannot be disposed of in a permitted landfill, alternative disposal methods will be proposed to the KDHE for review.

- Water IDW will be stored on-site in 55-gal drums or polyurethane containers. If acceptable to the KDHE, the wastewater may be aerated prior to sampling. Then the wastewater will be analyzed for VOCs, nitrate, and 1,2-dibromoethane by a KDHE-approved laboratory. The analysis for 1,2-dibromoethane will use EPA Method 504.1 or EPA Method SW-846 
8011 , to achieve the low detection limit of $0.05 \mu \mathrm{g} / \mathrm{L}$ (the maximum contaminant level for 1,2-dibromoethane).

- The results of the wastewater analyses will be discussed with the KDHE project manager, and the appropriate disposal method will be determined.

\subsection{Sampling and Reporting Schedule}

The proposed investigation is scheduled for 2007, pending successful negotiation of access. The CCC/USDA and Argonne will notify the KDHE a minimum of two weeks before field activities related to the proposed sampling program begin.

A report will be completed and submitted to the KDHE within 120 days after Argonne completes its quality review of the data. The report will follow the guidelines in Scope of Work (SOW) for a Comprehensive Investigation, established by KDHE Policy BER-RS-018 (KDHE 2005). Accordingly, the report will include, at a minimum, the following:

- A narrative of work conducted

- Recommendations for further action(s) at this site, if warranted

- Maps depicting sample locations, groundwater flow, and contaminant levels

- Tables that include all analytical and field data

- Laboratory analytical data reports

- Field documentation

- Quality assurance and quality control data 


\subsection{Quality Assurance and Quality Control}

Included in this section is a summary of methods that will be followed to meet quality assurance/quality control (QA/QC) standards. Descriptions of the QA/QC methods to monitor sampling and analysis are in Section 4.2 of the Master Work Plan (Argonne 2002). That document should be consulted for more a more detailed narrative of the QA/QC procedures.

The $\mathrm{QA} / \mathrm{QC}$ requirements during field sampling are as follows:

- Ensure that samples collected are representative of current site conditions.

- Ensure that sufficient samples are collected to meet the investigational goals.

- Ensure that field instrument calibration procedures are followed and that the appropriate number of field blanks, rinsate samples, trip blanks, and field replicates are collected. For this project, a minimum of one field blank, one rinsate sample, one trip blank, and one or two field replicates will be collected.

- Record in a bound notebook with printed page numbers all details of the work conducted. Use permanent ink for this documentation.

- To the extent possible, use disposable sampling equipment at each sampling location.

- Between wells, thoroughly rinse RediFlo pumps and hoses. Triple-wash all other nondisposable sampling equipment with a nonionic detergent in water, then rinse with water.

- Collect soil and groundwater samples according to the procedures specified in Section 3.3.

- Label sample containers as instructed in Appendix D, Section D.1.4, of the Master Work Plan (Argonne 2002). At a minimum, include the following 
information: sample identifier, date, time, preservative, and intended analysis. Use preprinted sample labels for this task.

- Complete a preprinted chain-of-custody record as instructed in Appendix D, Section D.1.5, of the Master Work Plan (Argonne 2002).

- Appropriately pack and seal shipping containers to ensure that chain of custody is maintained.

- Use preprinted shipping labels for sample containers being sent to laboratories for off-site analyses.

Laboratory $\mathrm{QA} / \mathrm{QC}$ procedures are designed to ensure that sample integrity is maintained and that sample analysis is reproducible. This will be accomplished, in part, by verifying that laboratory-related field documentation is complete and that procedures have been followed with regard to chain-of-custody records, sample storage, and sample holding times. In addition, laboratory procedures, equipment calibration, and performance standards (reproducibility, standards, spikes recoveries, etc.) will be reviewed and documented in the monitoring report discussed in Section 3.4.

Groundwater samples received at the AGEM Laboratory will be analyzed within the specified holding time for VOCs, including carbon tetrachloride and chloroform, according to a modification of EPA Method 524.2 (EPA 1995). Analysis for 1,2-dibromoethane will use EPA Method SW-846 8260B or EPA Method 524.2. To ensure reproducibility, duplicates of $10 \%$ of the samples (or a minimum of one sample) will be shipped directly from the field to a reference laboratory for analysis with the EPA's Contract Laboratory Program methods.

Soil samples received at the AGEM Laboratory will be subjected to purge-and-trap preparation, then analysis for carbon tetrachloride and chloroform using methanol extraction and a gas chromatograph-mass spectrometer system (EPA Methods 5030B and 8260B; EPA 1998), in accordance with the Master Work Plan (Argonne 2002). To ensure reproducibility, $10 \%$ of the samples will be selected for analysis by a second laboratory with the same analytical methodology. 
Quality assurance records completed during the project will be maintained by the QA/QC coordinator and stored with the project files.

\subsection{Health and Safety Information}

The general health and safety plan for the Hilton site is in Section 3 of the Master Work Plan (Argonne 2002). The general plan addresses all anticipated safety issues for the investigation. Specific emergency information for use at the site is given below.

Hilton has emergency 911 service. All emergency calls, including police, fire, and ambulance calls, will be directed for an appropriate response from this number. Note that 911 calls from cell phones can be routed to various 911 centers, depending on which tower picks up the call. Ask whether you have McPherson County Communications before you describe your emergency. The call will be transferred if you have reached a different 911 center.

No emergency medical facilities exist at Hilton. The nearest hospital with emergency medical facilities is in McPherson, Kansas. Driving directions to the hospital and the map showing the route are in Figure 3.4. Additional emergency information is in Table 3.2.

\subsection{Area Contacts}

Susan R. Meng, McPherson County Clerk

117 North Maple Street

McPherson, KS 67460

620-241-3656

Hours: 8 a.m. to 5 p.m.

U.S. Post Office

115 East Kansas Avenue

McPherson, KS 67460

620-241-1532 


\subsection{Argonne Contacts}

Lorraine M. LaFreniere, Ph.D.

Manager, Applied Geosciences and

Environmental Management Section

Environmental Science Division

9700 South Cass Avenue

Argonne, IL 60439-4843

630-252-7969

lafreniere@anl.gov

Y. Eugene Yan, Ph.D.

Hilton Project Manager

Environmental Science Division

9700 South Cass Avenue

Argonne, IL 60439-4843

630-252-6322

eyan@anl.gov
James Hansen

Community Relations Representative Environmental Science Division

Argonne National Laboratory 955 L'Enfant Plaza SW, Suite 6000

Washington, DC 20024

202-488-2453

hansenj@anl.gov 
TABLE 3.2 Emergency information for the Hilton, Kansas, investigation. ${ }^{a}$

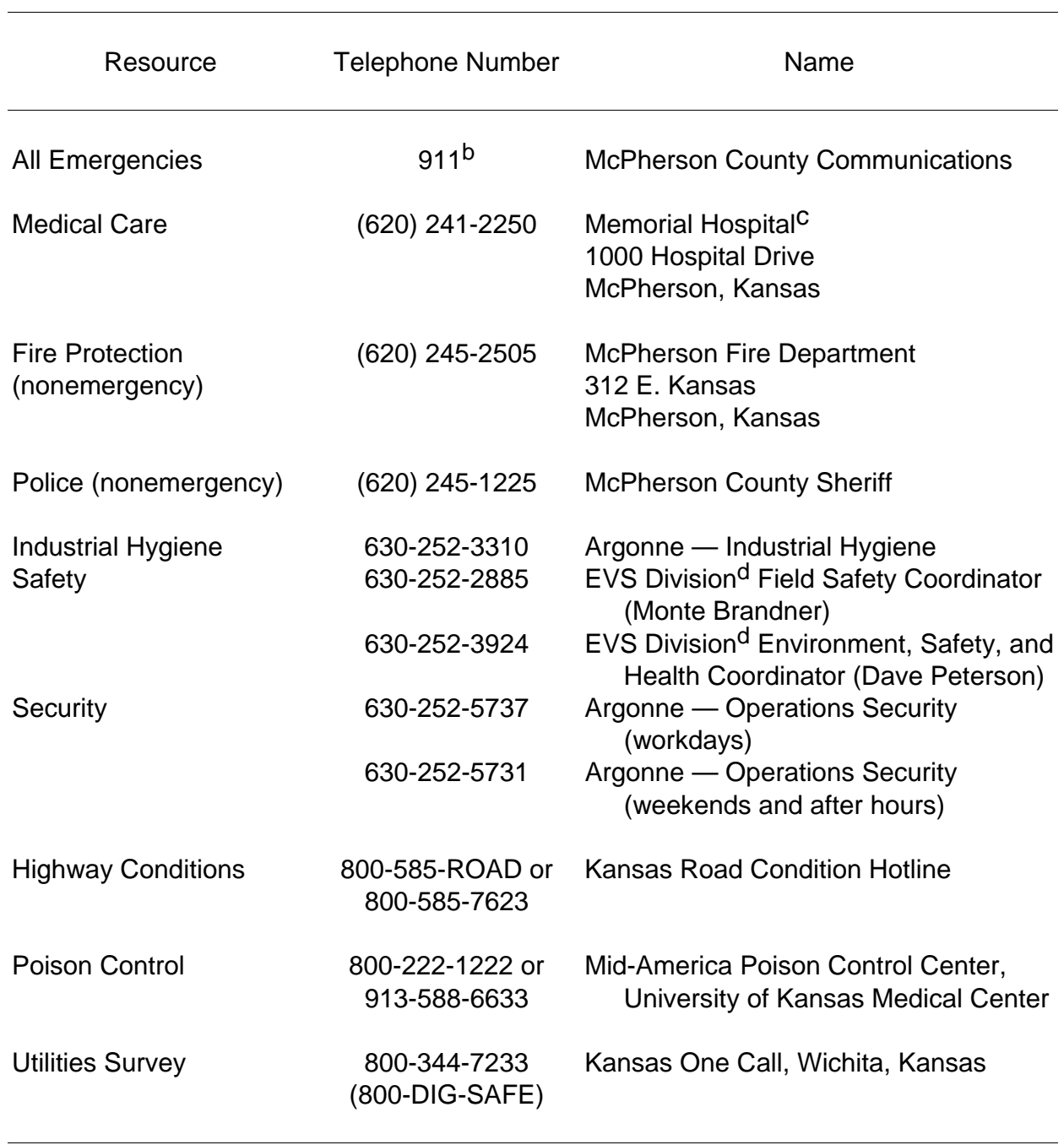

a post this table in the field operations base.

b 911 calls from cell phones can be picked up by various 911 centers, depending on call volumes. Ask whether you have the McPherson County Communications before you describe your emergency. The call will be transferred if you have reached a different 911 center.

C The route from Hilton to the McPherson Memorial Hospital is shown in Figure 3.4.

d Environmental Science Division at Argonne. 


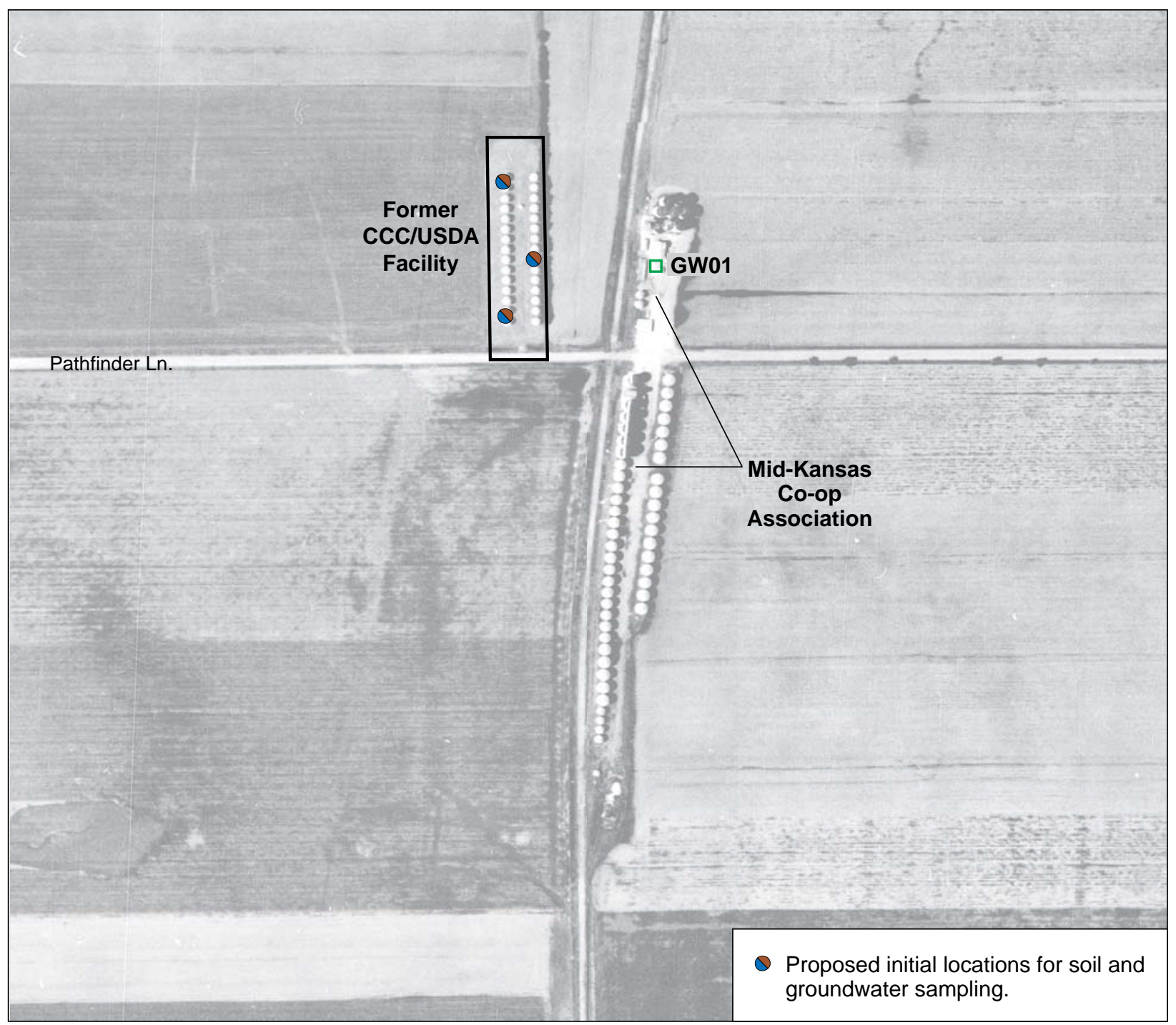

FIGURE 3.1 Proposed investigation locations on the former CCC/USDA property. Source of photograph: ASCS (1963). 


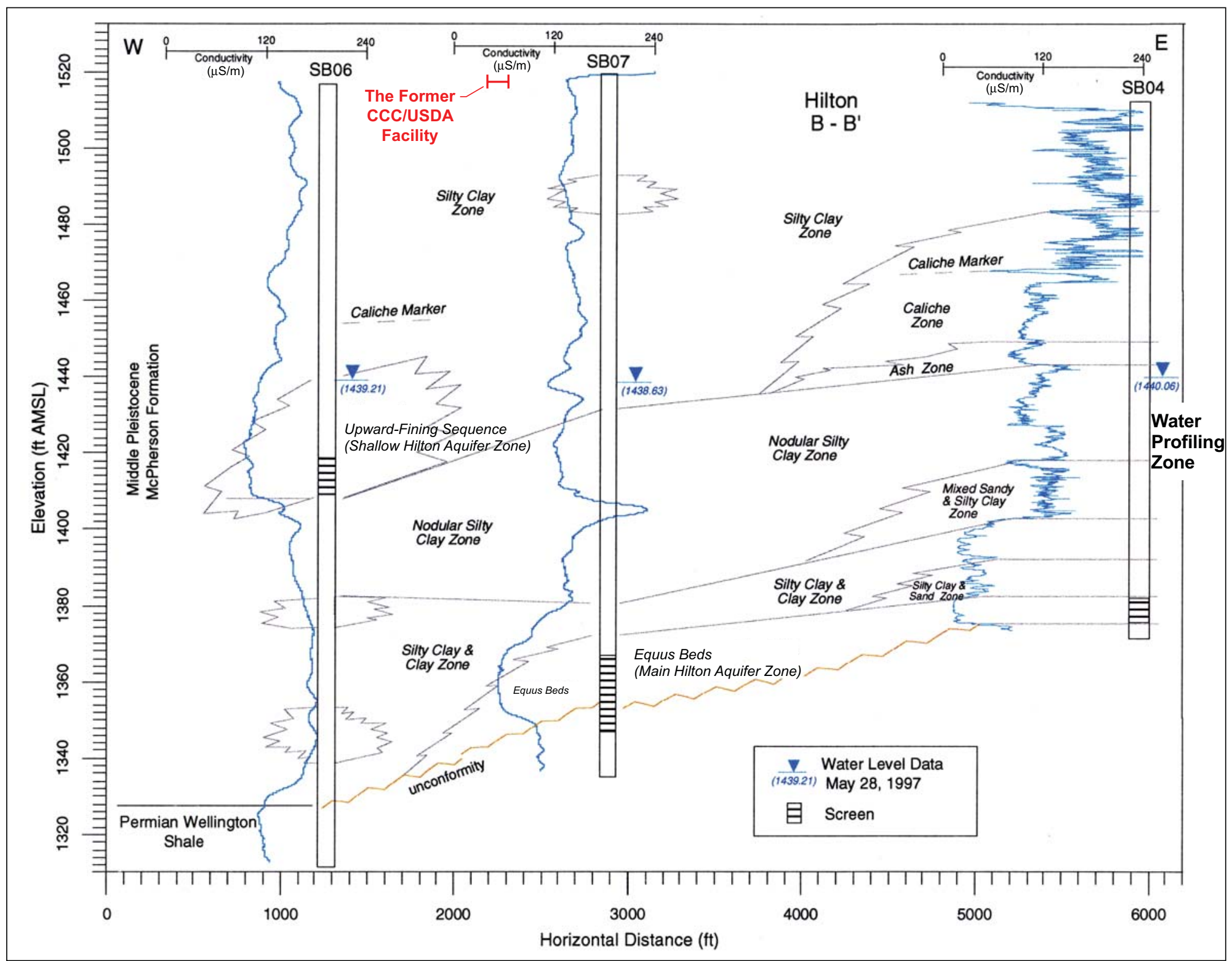

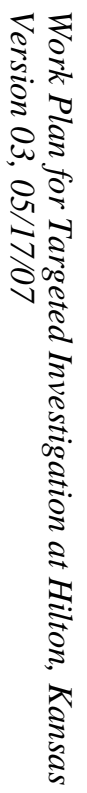

FIGURE 3.2 Interpreted hydrologic section B-B' (vertically exaggerated) through the former CCC/USDA grain storage facility. 


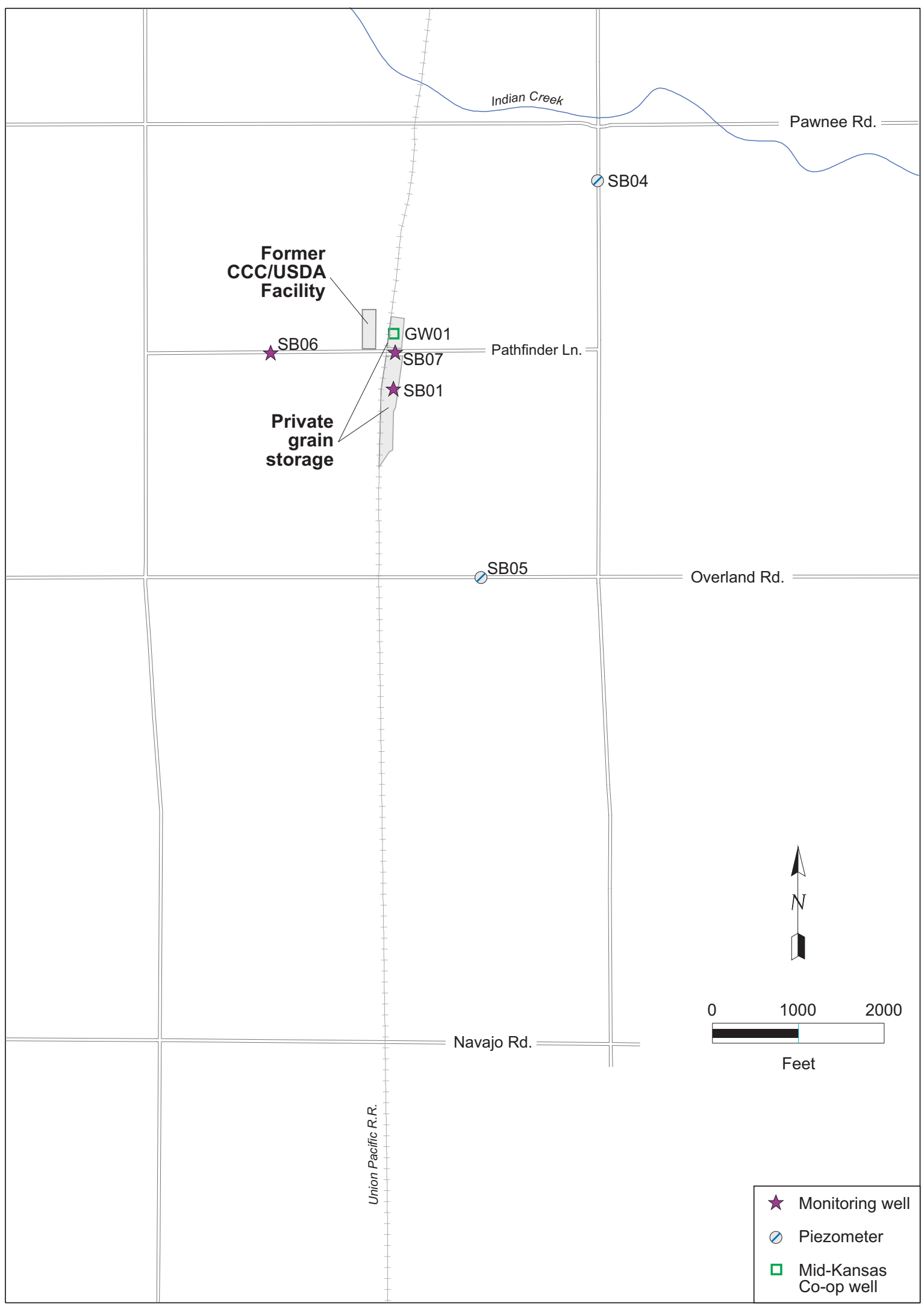

FIGURE 3.3 Location of existing monitoring wells, piezometers, and well GW01. 


\section{Directions from Hilton, Kansas, to Memorial Hospital, McPherson, Kansas (1000 Hospital Drive, McPherson, Kansas)}

- Follow Pathfinder Lane east $(0.45 \mathrm{mi})$ to 14 th Street (CR 1961).

- Follow 14th Street south $(2.7 \mathrm{mi})$, then bear left to North Main Street.

- Follow North Main Street south $(1.6 \mathrm{mi})$ to 4 th Street, then turn west (right).

- Follow 4th Street (west) for 3 blocks.

- Go left (south) just before Hospital Drive.

- Entrance to Emergency Room and Main Entrance (visitors) are next to each other and clearly marked.

\section{Emergency Route To McPherson Memorial Hospital}

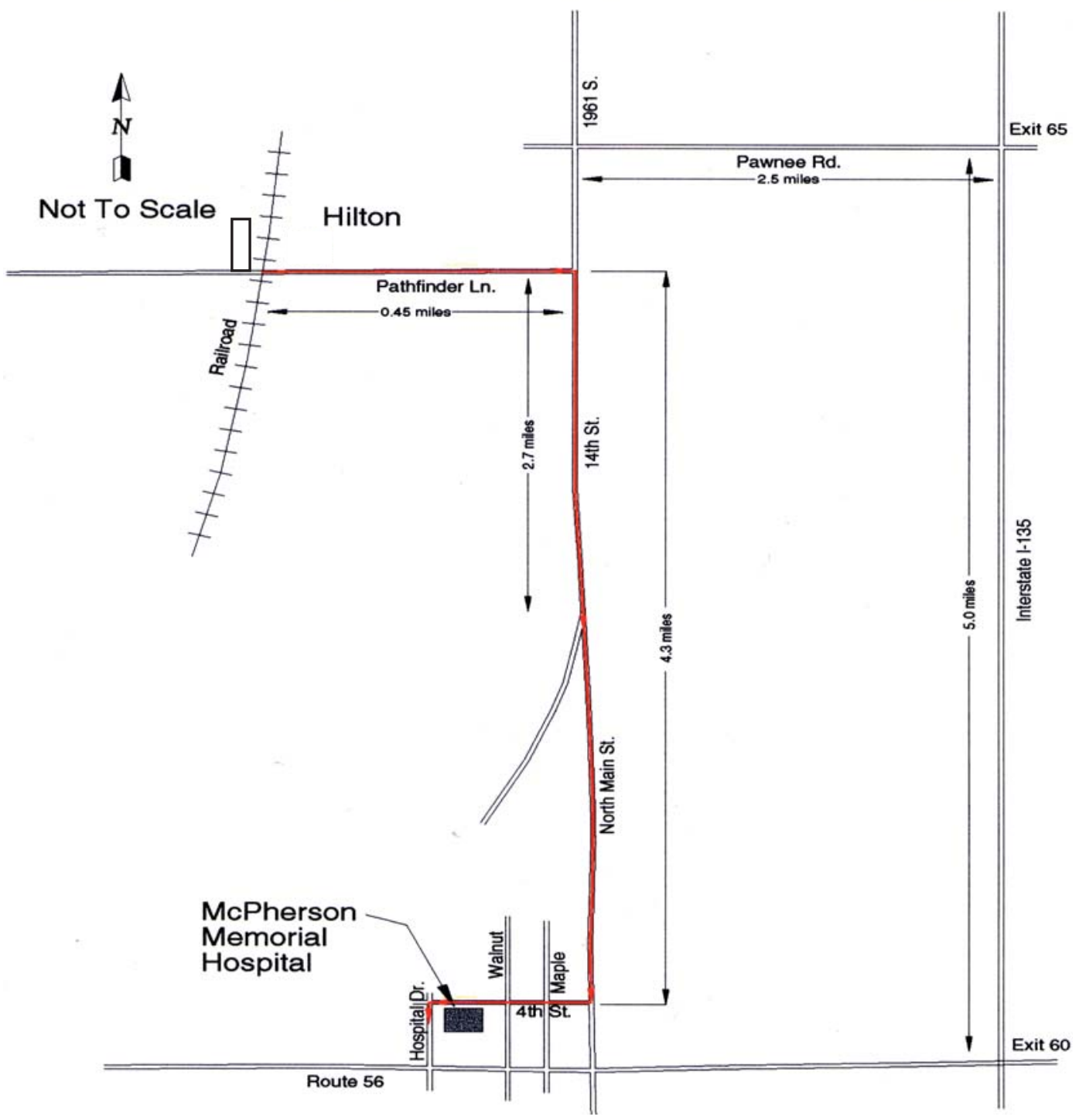

FIGURE 3.4 Emergency route from Hilton to Memorial Hospital, McPherson, Kansas. 


\section{References}

American Trails, 2003, "Rails to Trails: Court Denies Challenge to Financial Responsibility of

Non-Profit Trail Group," $\quad \underline{\text { http://www.americantrails.org/resources/railtrails/ }}$ RailChallengeFinancial.html, accessed February 19, 2007.

Argonne, 1997a, Final Phase I Report and Phase II Work Plan: Expedited Site Characterization, Hilton, Kansas, prepared for the Commodity Credit Corporation, U.S. Department of Agriculture, Washington, D.C., by Argonne National Laboratory, Argonne, Illinois, June.

Argonne, 1997b, Final Phase II Report: Expedited Site Characterization, Hilton, Kansas, prepared for the Commodity Credit Corporation, U.S. Department of Agriculture, Washington, D.C., by Argonne National Laboratory, Argonne, Illinois, October.

Argonne, 2002, Final Master Work Plan: Environmental Investigations at Former CCC/USDA Facilities in Kansas, 2002 Revision, ANL/ER/TR-02/004, prepared for the Commodity Credit Corporation, U.S. Department of Agriculture, Washington, D.C., by Argonne National Laboratory, Argonne, Illinois, December.

ASCS, 1950, aerial photo of Hilton, Kansas (site KA-ZZ, pic 93725, frame 134), U.S. Department of Agriculture, Agricultural Stabilization and Conservation Service, Aerial Photography Field Office, Salt Lake City, Utah, September 6.

ASCS, 1956, aerial photo of Hilton, Kansas (site KA-ZZ, pic 93725, frame 13), U.S. Department of Agriculture, Agricultural Stabilization and Conservation Service, Aerial Photography Field Office, Salt Lake City, Utah, June 9.

ASCS, 1963, aerial photo of Hilton, Kansas (site KA-ZZ, pic 93725, frame 157), U.S. Department of Agriculture, Agricultural Stabilization and Conservation Service, Aerial Photography Field Office, Salt Lake City, Utah, July 18.

ASCS, 1970, aerial photo of Hilton, Kansas (site KA-ZZ, pic 93725, frame 75), U.S. Department of Agriculture, Agricultural Stabilization and Conservation Service, Aerial Photography Field Office, Salt Lake City, Utah, June 27. 
Carey, C., 2005, analytical results provided by Carey (Bureau of Environment Remediation, Kansas Department of Health and Environment, Topeka, Kansas) in attachment to letter to L. LaFreniere (Argonne National Laboratory, Argonne, Illinois), August 25.

EPA, 1995, Method 524.2: Measurement of Purgeable Organic Compounds in Water by Capillary Column Gas Chromatography/Mass Spectrometry, Revision 4.1, edited by J.W. Munch, National Exposure Research Laboratory, Office of Research and Development, U.S. Environmental Protection Agency, Cincinnati, Ohio.

EPA, 1998, Test Methods for Evaluating Solid Waste: Physical/Chemical Methods, EPA SW-846, 3rd edition, Draft Update IVA, U.S. Environmental Protection Agency, January.

Gurwitz, M., 1998, letter from Gurwitz (Office of the General Counsel, U.S. Department of Agriculture, Washington, D.C.) to J. Kahn (Assistant Regional Counsel, U.S. Environmental Protection Agency, Region VII, Kansas City, Kansas), regarding a request for access assistance for investigations at Hilton and Ramona, Kansas, July 27.

Kahn, J., 1998, letter from Kahn (Assistant Regional Counsel, U.S. Environmental Protection Agency, Region VII, Kansas City, Kansas), to M. Gurwitz (Office of the General Counsel, U.S. Department of Agriculture, Washington, D.C.), regarding a request for access assistance for investigations at Hilton and Ramona, Kansas, December 18.

KDHE, 1993, Preliminary Assessment - Hilton Grain Bins Site, Hilton, Kansas, KSD985015320, Bureau of Environment Remediation, Kansas Department of Health and Environment, Topeka, Kansas, March.

KDHE, 1994, Screening Site Inspection, Hilton Grain Bins Site, Hilton, Kansas, KSD985015320, Bureau of Environment Remediation, Kansas Department of Health and Environment, Topeka, Kansas, September

KDHE, 1995, Draft Consent Order for the Hilton Grain Bins Site, McPherson County, Kansas, Kansas Department of Health and Environment, Topeka, Kansas.

KDHE, 2005, Scope of Work (SOW) for a Comprehensive Investigation, BER Policy \#BER-RS018, Bureau of Environmental Remediation/Remedial Section Guidance, Kansas Department of 
Health and Environment, Topeka, Kansas, revised 2005 (http://www.kdheks.gov/ber/policies/ BER_RS_018_SOW.pdf).

KGS, 1997, electronic file of regional water level measurements for January 1997, Kansas Geological Survey, Lawrence, Kansas (http://www.kgs.ku.edu).

NAIP, 2002, aerial photograph of Hilton, Kansas, National Agricultural Imagery Program, U.S. Department of Agriculture, Washington, D.C. (http://www.apfo.USDA.gov/NAIP.html). 


\section{Appendix A}

Comprehensive Historical Analytical Data for Hilton, Kansas 
TABLE A.1 Analytical results for previous sampling at Hilton, Kansas.

Concentration (VOCs Units)

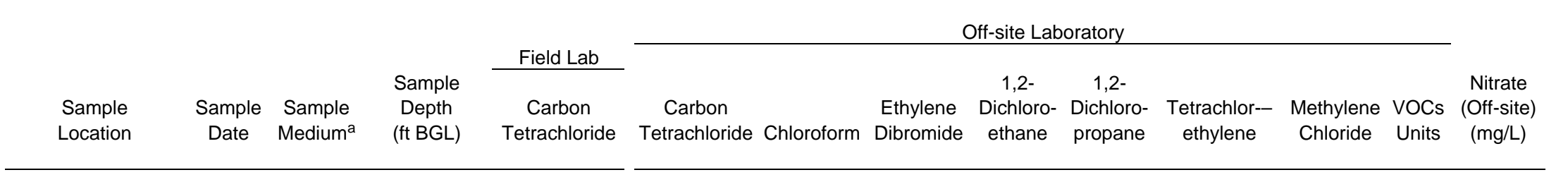

1992 Groundwater Sampling (KDHE 1993)

\begin{tabular}{|c|c|c|c|c|c|c|c|c|c|c|c|c|c|}
\hline Co-op (GW01) & $5 / 12 / 92$ & GW & $166(T D)^{b}$ & $-c$ & 910 & $N A^{d}$ & 2.9 & NA & NA & NA & NA & $\mu \mathrm{g} / \mathrm{L}$ & 88 \\
\hline Case (DW06) & $11 / 30 / 92$ & GW & 175 (TD) & - & $\mathrm{ND}^{\mathrm{e}}(2)$ & ND (2) & ND (2) & NA & NA & NA & NA & $\mu \mathrm{g} / \mathrm{L}$ & NA \\
\hline Davis (DW02) & $11 / 30 / 92$ & GW & Unknown & - & ND (2) & ND (2) & ND (2) & NA & NA & NA & NA & $\mu \mathrm{g} / \mathrm{L}$ & NA \\
\hline Houchen (DW04) & $11 / 30 / 92$ & GW & Unknown & - & ND (2) & ND (2) & ND (2) & NA & NA & NA & NA & $\mu \mathrm{g} / \mathrm{L}$ & NA \\
\hline Ledell (shallow) & $11 / 30 / 92$ & GW & Unknown & - & ND (2) & ND (2) & ND (2) & NA & NA & NA & NA & $\mu \mathrm{g} / \mathrm{L}$ & NA \\
\hline Ledell (deep) & $11 / 30 / 92$ & GW & Unknown & - & ND (2) & ND (2) & ND (2) & NA & NA & NA & NA & $\mu \mathrm{g} / \mathrm{L}$ & NA \\
\hline Musselwhite & $11 / 30 / 92$ & GW & Unknown & - & ND (2) & ND (2) & ND (2) & NA & NA & NA & NA & $\mu \mathrm{q} / \mathrm{L}$ & NA \\
\hline
\end{tabular}

1994 Soil and Groundwater Sampling (KDHE 1994)

$\begin{array}{lcccc}\text { S-1 } & 8 / 31 / 94 & \text { Soil } & 8 & \text { ND }(0.1) \\ \text { S-2 } & 8 / 31 / 94 & \text { Soil } & 8 & \text { ND }(0.1) \\ \text { S-3 } & 8 / 31 / 94 & \text { Soil } & 8 & \text { ND }(0.1) \\ \text { S-4 } & 8 / 31 / 94 & \text { Soil } & 8 & \text { ND }(0.1) \\ \text { S-5 } & 8 / 31 / 94 & \text { Soil } & 8 & 0.16 \\ \text { S-6 } & 8 / 31 / 94 & \text { Soil } & 8 & \text { ND }(0.1) \\ \text { S-7 } & 8 / 31 / 94 & \text { Soil } & 8 & \text { ND }(0.1) \\ \text { S-8 } & 8 / 31 / 94 & \text { Soil } & 8 & \text { ND }(0.1) \\ \text { S-9 } & 9 / 1 / 94 & \text { Soil } & 17 & 0.26 \\ \text { S-10 } & 9 / 1 / 94 & \text { Soil } & 17 & 0.13 \\ & & & & \end{array}$

1996 Groundwater, Soil, and Vegetation Sampling (Argonne 1997a)

\begin{tabular}{|c|c|c|c|c|c|c|c|c|c|c|c|c|c|}
\hline Case (DW06) & $8 / 28 / 96$ & GW & 175 (TD) & - & ND (5) & ND (5) & NA & NA & NA & NA & NA & $\mu \mathrm{g} / \mathrm{L}$ & $<0.1$ \\
\hline Davis (DW02) & 8/27/96 & GW & Unknown & - & ND (5) & ND (5) & NA & NA & NA & NA & NA & $\mu \mathrm{g} / \mathrm{L}$ & 0.5 \\
\hline Hein (DW05) & 8/27/96 & GW & $\sim 190$ & - & ND (5) & ND (5) & NA & NA & NA & NA & NA & $\mu \mathrm{g} / \mathrm{L}$ & 0.2 \\
\hline Houchen (DW04) & 8/27/96 & GW & $\sim 90$ & - & ND (5) & ND (5) & NA & NA & NA & NA & NA & $\mu \mathrm{g} / \mathrm{L}$ & $<0.1$ \\
\hline
\end{tabular}


Concentration (VOCs Units)

Off-site Laboratory

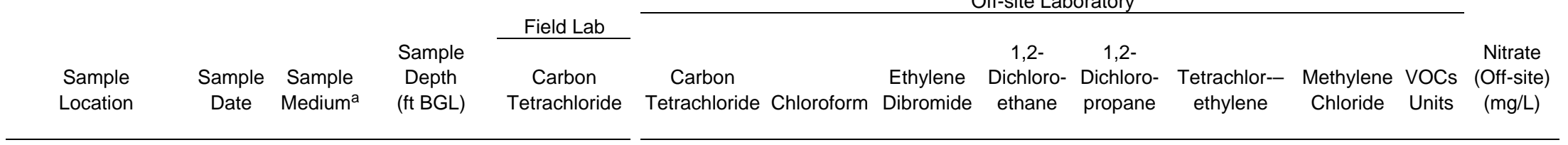

1996 Groundwater, Soil, and Vegetation Sampling (Argonne 1997a) (cont.)

\begin{tabular}{|c|c|c|c|c|c|c|c|c|c|c|c|c|c|}
\hline Hunt (DW03) & $8 / 27 / 96$ & GW & Unknown & - & ND (5) & ND (5) & NA & NA & NA & NA & NA & $\mu \mathrm{g} / \mathrm{L}$ & 0.5 \\
\hline Johnson (DW01) & $8 / 27 / 96$ & GW & Unknown & - & ND (5) & ND (5) & NA & NA & NA & NA & NA & $\mu \mathrm{g} / \mathrm{L}$ & 2.5 \\
\hline Co-op (GW01) & $8 / 28 / 96$ & GW & 166 (TD) & - & 800 & 42 & NA & NA & NA & NA & NA & $\mu \mathrm{g} / \mathrm{L}$ & $<0.1$ \\
\hline SB03 & 10/10/96 & GW & 159.7-162.7 & - & ND (5) & ND (5) & NA & NA & NA & NA & NA & $\mu \mathrm{g} / \mathrm{L}$ & 0.2 \\
\hline SB04 & 9/4/96 & GW & $130-135$ & - & ND (5) & ND (5) & NA & NA & NA & NA & NA & $\mu \mathrm{g} / \mathrm{L}$ & 0.3 \\
\hline SB05 & 9/5/96 & GW & 138-148 & - & ND (5) & ND (5) & NA & NA & NA & NA & NA & $\mu \mathrm{g} / \mathrm{L}$ & 0.7 \\
\hline 087S/000E & 9/5/96 & Soil & $0.8-0.9$ & - & ND (30) & ND (30) & NA & NA & NA & NA & NA & $\mathrm{pg} / \mathrm{g}$ & NA \\
\hline 119S/000E & 9/5/96 & Soil & $0.8-0.9$ & - & 108 & ND (30) & NA & NA & NA & NA & NA & $\mathrm{pg} / \mathrm{g}$ & NA \\
\hline 150S/000E & 9/5/96 & Soil & $0.8-0.9$ & - & 400 & ND (30) & NA & NA & NA & NA & NA & $\mathrm{pg} / \mathrm{g}$ & NA \\
\hline 180S/000E & 9/5/96 & Soil & $0.8-0.9$ & - & $<30$ & ND (30) & NA & NA & NA & NA & NA & $\mathrm{pg} / \mathrm{g}$ & NA \\
\hline 210S/000E & 9/5/96 & Soil & $0.8-0.9$ & - & 68.5 & ND (30) & NA & NA & NA & NA & NA & $\mathrm{pg} / \mathrm{g}$ & NA \\
\hline $242 S / 000 E$ & 9/5/96 & Soil & $0.8-0.9$ & - & $<30$ & ND (30) & NA & NA & NA & NA & NA & $\mathrm{pg} / \mathrm{g}$ & NA \\
\hline $292 S / 000 E$ & 9/5/96 & Soil & $1.0-1.1$ & - & ND (30) & ND (30) & NA & NA & NA & NA & NA & $\mathrm{pg} / \mathrm{g}$ & NA \\
\hline $323 S / 000 E$ & 9/5/96 & Soil & $0.8-0.9$ & - & ND (30) & ND (30) & NA & NA & NA & NA & NA & $\mathrm{pg} / \mathrm{g}$ & NA \\
\hline $355 \mathrm{~S} / 000 \mathrm{E}$ & 9/5/96 & Soil & $0.8-0.9$ & - & ND (30) & ND (30) & NA & NA & NA & NA & NA & $\mathrm{pg} / \mathrm{g}$ & NA \\
\hline 475S/000E & 9/5/96 & Soil & $0.8-0.9$ & - & ND (30) & ND (30) & NA & NA & NA & NA & NA & $\mathrm{pg} / \mathrm{g}$ & NA \\
\hline 508S/000E & 9/5/96 & Soil & $0.8-0.9$ & - & ND (30) & ND (30) & NA & NA & NA & NA & NA & $\mathrm{pg} / \mathrm{g}$ & NA \\
\hline $538 \mathrm{~S} / 000 \mathrm{E}$ & 9/5/96 & Soil & $0.8-0.9$ & - & ND (30) & ND (30) & NA & NA & NA & NA & NA & $\mathrm{pg} / \mathrm{g}$ & NA \\
\hline 570S/000E & 9/5/96 & Soil & $0.8-0.9$ & - & ND (30) & ND (30) & NA & NA & NA & NA & NA & $\mathrm{pg} / \mathrm{g}$ & NA \\
\hline 600S/000E & 9/5/96 & Soil & $0.8-0.9$ & - & ND (30) & ND (30) & NA & NA & NA & NA & NA & $\mathrm{pg} / \mathrm{g}$ & NA \\
\hline 056S/000E & 9/4/96 & Veg & None & - & ND (30) & ND (30) & NA & NA & NA & NA & NA & $\mathrm{pg} / \mathrm{g}$ & NA \\
\hline 087S/000E & 9/4/96 & Veg & None & - & ND (30) & ND (30) & NA & NA & NA & NA & NA & $\mathrm{pg} / \mathrm{g}$ & NA \\
\hline 119S/000E & 9/4/96 & Veg & None & - & ND (30) & ND (30) & NA & NA & NA & NA & NA & $\mathrm{pg} / \mathrm{g}$ & NA \\
\hline $150 S / 000 E$ & 9/4/96 & Veg & None & - & ND (30) & ND (30) & NA & NA & NA & NA & NA & $\mathrm{pg} / \mathrm{g}$ & NA \\
\hline
\end{tabular}


1996 Groundwater, Soil, and Vegetation Sampling (Argonne 1997a) (cont.)

\begin{tabular}{|c|c|c|c|c|c|c|c|c|c|c|c|c|c|}
\hline 180S/000E & $9 / 4 / 96$ & Veg & None & - & ND (30) & ND (30) & NA & NA & NA & NA & NA & $\mathrm{pg} / \mathrm{g}$ & NA \\
\hline $210 \mathrm{~S} / 000 \mathrm{E}$ & 9/4/96 & Veg & None & - & ND (30) & ND (30) & NA & NA & NA & NA & NA & $\mathrm{pg} / \mathrm{g}$ & NA \\
\hline $242 \mathrm{~S} / 000 \mathrm{E}$ & 9/4/96 & Veg & None & - & ND (30) & ND (30) & NA & NA & NA & NA & NA & $\mathrm{pg} / \mathrm{g}$ & NA \\
\hline $292 \mathrm{~S} / 000 \mathrm{E}$ & 9/4/96 & Veg & None & - & ND (30) & ND (30) & NA & NA & NA & NA & NA & $\mathrm{pg} / \mathrm{g}$ & NA \\
\hline $323 \mathrm{~S} / 000 \mathrm{E}$ & 9/4/96 & Veg & None & - & ND (30) & ND (30) & NA & NA & NA & NA & NA & $\mathrm{pg} / \mathrm{g}$ & NA \\
\hline 355S/000E & 9/4/96 & Veg & None & - & ND (30) & ND (30) & NA & NA & NA & NA & NA & $\mathrm{pg} / \mathrm{g}$ & NA \\
\hline 394S/000E & 9/4/96 & Veg & None & - & 34.1 & 55 & NA & NA & NA & NA & NA & $\mathrm{pg} / \mathrm{g}$ & NA \\
\hline $415 \mathrm{~S} / 000 \mathrm{E}$ & 9/4/96 & Veg & None & - & ND (30) & ND (30) & NA & NA & NA & NA & NA & $\mathrm{pg} / \mathrm{g}$ & NA \\
\hline $475 \mathrm{~S} / 000 \mathrm{E}$ & 9/4/96 & Veg & None & - & ND (30) & ND (30) & NA & NA & NA & NA & NA & $\mathrm{pg} / \mathrm{g}$ & NA \\
\hline $508 \mathrm{~S} / 000 \mathrm{E}$ & 9/4/96 & Veg & None & - & ND (30) & ND (30) & NA & NA & NA & NA & NA & $\mathrm{pg} / \mathrm{g}$ & NA \\
\hline 570S/000E & 9/4/96 & Veg & None & - & ND (30) & ND (30) & NA & NA & NA & NA & NA & $\mathrm{pg} / \mathrm{g}$ & NA \\
\hline $600 S / 000 E$ & 9/4/96 & Veg & None & - & ND (30) & ND (30) & NA & NA & NA & NA & NA & $\mathrm{pg} / \mathrm{g}$ & NA \\
\hline \multicolumn{14}{|c|}{ April 1997 Groundwater Sampling (Argonne 1997b) } \\
\hline Co-op (GW01) & $4 / 13 / 97$ & GW & 166 (TD) & - & 918 & 68 & NA & NA & NA & NA & NA & $\mu \mathrm{g} / \mathrm{L}$ & NA \\
\hline Co-op (GW01) & $4 / 13 / 97$ & GW & 166 (TD) & - & 887 & 60 & NA & NA & NA & NA & NA & $\mu \mathrm{g} / \mathrm{L}$ & NA \\
\hline Co-op (GW01) & $4 / 14 / 97$ & GW & 166 (TD) & - & 910 & 52 & NA & NA & NA & NA & NA & $\mu \mathrm{g} / \mathrm{L}$ & NA \\
\hline SB07 & $4 / 23 / 97$ & GW & $146.5-166.5$ & & ND (5) & ND (5) & NA & NA & NA & NA & NA & $\mu \mathrm{g} / \mathrm{L}$ & 0.4 \\
\hline \multicolumn{14}{|c|}{ October 1997 Groundwater Sampling by Argonne } \\
\hline Co-op (GW01) & $10 / 7 / 97$ & GW & 166 (TD) & - & 1100 & 48 & NA & NA & NA & NA & NA & $\mu \mathrm{g} / \mathrm{L}$ & NA \\
\hline SB07 & $10 / 9 / 97$ & GW & $146.5-166.5$ & - & ND (2) & ND (2) & NA & ND (2) & ND (2) & ND (2) & ND (2) & $\mu \mathrm{g} / \mathrm{L}$ & NA \\
\hline
\end{tabular}




\begin{tabular}{|c|c|c|c|c|c|c|c|c|c|c|c|c|c|}
\hline \multirow[b]{3}{*}{$\begin{array}{l}\text { Sample } \\
\text { Location }\end{array}$} & \multirow[b]{3}{*}{$\begin{array}{l}\text { Sample } \\
\text { Date }\end{array}$} & \multirow[b]{3}{*}{$\begin{array}{l}\text { Sample } \\
\text { Medium }^{\mathrm{a}}\end{array}$} & \multirow[b]{3}{*}{$\begin{array}{l}\text { Sample } \\
\text { Depth } \\
\text { (ft BGL) }\end{array}$} & \multicolumn{9}{|c|}{ Concentration (VOCs Units) } & \multirow[b]{3}{*}{$\begin{array}{c}\text { Nitrate } \\
\text { (Off-site) } \\
\text { (mg/L) }\end{array}$} \\
\hline & & & & \multirow{2}{*}{$\begin{array}{c}\text { Field Lab } \\
\text { Carbon } \\
\text { Tetrachloride }\end{array}$} & \multicolumn{8}{|c|}{ Off-site Laboratory } & \\
\hline & & & & & $\begin{array}{c}\text { Carbon } \\
\text { Tetrachloride }\end{array}$ & Chloroform & $\begin{array}{l}\text { Ethylene } \\
\text { Dibromide }\end{array}$ & $\begin{array}{c}1,2- \\
\text { Dichloro- } \\
\text { ethane }\end{array}$ & $\begin{array}{c}\text { 1,2- } \\
\text { Dichloro- } \\
\text { propane }\end{array}$ & $\begin{array}{c}\text { Tetrachlor-- } \\
\text { ethylene }\end{array}$ & $\begin{array}{l}\text { Methylene } \\
\text { Chloride }\end{array}$ & $\begin{array}{l}\text { VOCs } \\
\text { Units }\end{array}$ & \\
\hline \multicolumn{14}{|c|}{2005 Private Well Sampling by the KDHE (Carey 2005) } \\
\hline Davis (DW02) & 8/3/05 & GW & Unknown & & ND $(0.7)$ & ND (0.5) & ND (0.04) & ND (0.6) & ND (0.4) & ND (1.1) & ND (0.9) & $\mu g / L$ & NA \\
\hline Decker & 8/3/05 & GW & Unknown & - & ND (0.7) & ND (0.5) & ND (0.04) & ND (0.6) & ND (0.4) & ND (1.1) & ND (0.9) & $\mu \mathrm{g} / \mathrm{L}$ & NA \\
\hline Driskell & 8/3/05 & GW & Unknown & - & ND (0.7) & ND (0.5) & ND (0.04) & ND (0.6) & ND (0.4) & ND (1.1) & ND (0.9) & $\mu \mathrm{g} / \mathrm{L}$ & NA \\
\hline Houchen (DW04) & 8/3/05 & GW & $\sim 90$ & - & ND (0.7) & ND $(0.5)$ & ND (0.04) & ND (0.6) & ND (0.4) & ND (1.1) & ND (0.9) & $\mu \mathrm{g} / \mathrm{L}$ & NA \\
\hline \multicolumn{14}{|l|}{ Johnson } \\
\hline (Pawnee Rd.) & $8 / 3 / 05$ & GW & Unknown & - & ND $(0.7)$ & $\mathrm{ND}(0.5)$ & ND (0.04) & ND (0.6) & ND $(0.4)$ & ND (1.1) & ND (0.9) & $\mu \mathrm{g} / \mathrm{L}$ & NA \\
\hline Ledell & $8 / 3 / 05$ & GW & Unknown & - & ND $(0.7)$ & ND (0.5) & ND (0.04) & ND (0.6) & ND (0.4) & ND (1.1) & ND (0.9) & $\mu \mathrm{g} / \mathrm{L}$ & NA \\
\hline
\end{tabular}

a Sample medium: GW, groundwater; Veg, vegetation.

b TD, total depth.

c Sample not submitted to the indicated field or off-site laboratory for analysis.

d NA, not analyzed.

e ND, not detected at the indicated quantitation limit. 


\section{Appendix B:}

Records of Property Transactions Affecting the Grain Storage Facilities at Hilton, Kansas 
TABLE B.1 Timeline of Hilton property documentation on file at Argonne. ${ }^{a}$

Date

Item

Property Description

Property Transfer Records

6/17/1898 Sellberg to Olson

8/30/1899 Sellberg to railroad

11/29/1899 Blomberg to railroad

6/3/1904 Sellberg to railroad

$12 / 22 / 1941$

Blomberg to Howse

12/27/1941 Sellberg to Sellberg
Deed record for the strip of land in the Southeast Quarter of Section 32, Township 18 South, Range 3 West, located west of the Union Pacific Railroad; approximately two acres no longer used as a public highway.

A strip and tract of land in the Southeast Quarter of Section 32, T18S, R3W, located east of the railroad. The tract of land is described in detail with dimensions relative to the railroad centerline and the northern line of the quarter (Pathfinder Lane). Commencing at a point $33 \mathrm{ft}$ east of the centerline of the main track of the Salina and Southwest Railway and $16.5 \mathrm{ft}$ south of the north line of the quarter, then southwest $303.5 \mathrm{ft}$ along the railroad right-of-way to the Hilton Stock Yards, then southeast $100 \mathrm{ft}$, then northeast $318.5 \mathrm{ft}$ along a line that is $133 \mathrm{ft}$ east of the centerline of the main track, then west $100 \mathrm{ft}$ to the point of beginning. (This land is future co-op property south of Pathfinder Lane.)

Tract of land in Northeast Quarter of Section 32, T18S, R3W, conveyed to railroad use. Commencing at a point $16 \mathrm{ft}$ north of the south line of the northeast quarter (Pathfinder Lane) and east of the Salina and Southwestern Railway right-of-way, then east $100 \mathrm{ft}$, then north $379.5 \mathrm{ft}$ along a line parallel to the right-ofway, then west $100 \mathrm{ft}$, then south $379.5 \mathrm{ft}$ to the point of beginning. (This land is future co-op property north of Pathfinder Lane.)

Additional land (1.92 acres) in the northwest quarter of the Southeast Quarter of Section 32, T18S, R3W, conveyed to railroad use. Position of this tract of land is said to be south of a tract of land deeded by Sellberg to the railroad on 12/19/1889 (document not on file at Argonne). Commencing at a point $470 \mathrm{ft}$ south of the east-west centerline of Section 32 (Pathfinder Lane) and $33 \mathrm{ft}$ east of the centerline of the main track of the Union Pacific Railroad, then east $100 \mathrm{ft}$ along the southern boundary of the earlier deed parcel, then south a distance of $830 \mathrm{ft}$ on a line $133 \mathrm{ft}$ east of the centerline of the main track to intersect with the south line of the northwest quarter of the southeast quarter of Section 32, then west a distance of $100 \mathrm{ft}$ on the south line of the northwest quarter of the southeast quarter of Section 32, then north $845 \mathrm{ft}$ to the point of beginning. (This land is additional future co-op property south of Pathfinder Lane.)

All of the Northeast Quarter of Section 32, T18S, R3W, except for two tracts of land: (1) the right-of-way of the Union Pacific Railroad, formerly the Salina and Southwestern Railway Co.; and (2) a parcel adjacent to the railway, $100 \mathrm{ft}$ by $379.5 \mathrm{ft}$, described in the 11/29/1899 document.

All of the Southeast Quarter of Section 32, T18S, R3W, lying east of the Union Pacific Railroad right-of-way. 
1/29/1959 Tector to McPherson Grain Co.

$4 / 15 / 1959$

$6 / 20 / 1963$

$4 / 30 / 1965$

$2 / 5 / 1973$

\section{extension from}

to $5 / 1 / 1964$

CCCIUSDA lease

extension from

to $4 / 30 / 1969$

\section{Termination of}

CCC/USDA lease

Ledell to Houchen
Item

Property Description

Pages

Northern half of the Northeast Quarter of Section 32, T18S, R3W, except the right-of-way of the Union

Pacific Railroad Company, formerly the Salina and Southwestern Railway Company. (The 12/9/1997

1

document transfers the southern half of the Northeast Quarter of Section 32.)

CCC/USDA lease from Beginning in the southeast corner of the Northwest Quarter of Section 32, T18S, R3W, from the center of K. Peterson for 5/1/1954 the public highway (Pathfinder Lane), then $510 \mathrm{ft}$ north, $128 \mathrm{ft}$ west, $510 \mathrm{ft}$ south, and $128 \mathrm{ft}$ east to point of to $5 / 1 / 1959$ beginning (approximately 1.5 acres)

The Southeast Quarter of Section 32, T18S, R3W, located east of the Union Pacific Railroad right-of-way (150 acres)

Property east of the railroad and south of the highway (Pathfinder Lane) deeded to the co-op. Commencing at the northwest corner of the Southeast Quarter in Section 32, T18S, R3W, then south 40 rods (or $660 \mathrm{ft}$ ) along the east margin of the Union Pacific right-of-way, then east 2 rods (or $33 \mathrm{ft}$ ), then north 40 rods (or $660 \mathrm{ft}$ ) to a point 2 rods (or $33 \mathrm{ft}$ ) east of the place of the beginning, then west 2 rods (or $33 \mathrm{ft}$ ) to the point of the beginning

\section{CCC/USDA lease $\quad$ Same as initial 1954 lease in the Northwest Quarter of Section 32, T18S, R3W}

K. Peterson for 5/1/1959

K. Peterson for 5/1/1964

Same as initial 1954 lease in the Northwest Quarter of Section 32, T18S, R3W.

Same as initial 1954 lease in the Northwest Quarter of Section 32, T18S, R3W.
Joint tenancy warranty deed for 4.44 acres of land in the Northeast Quarter of Section 32 and west of the railroad. Beginning at the northwest corner of the Northeast Quarter of Section 32, then east $499.7 \mathrm{ft}$ along the section line to the west line of the railroad right-of-way, then southwest $438 \mathrm{ft}$ along the right-of-way, then northwest $441.6 \mathrm{ft}$, then north $387 \mathrm{ft}$ to point of beginning. 
Property Transfer Records (cont.)

\section{8/1/1980 Ledell to Ledell}

Joint tenancy warranty deed for the northern half of the Northeast Quarter of Section 32, T18S, R3W, except for the land west of the railroad described in the 2/5/1973 document.

6/10/1992 Wall-Rogalsky Milling Co. Warranty deed transferring five tracts of land in McPherson County. Tract 4 is part of the Southeast Quarter to Mid-Kansas Co-op of Section 32, T18S, R3W, described in the 1/29/1959, 10/19/1981, and 12/29/1981 documents as property 40 rods by 2 rods, east of the railroad and south of the highway (Pathfinder Lane).

6/6/1997 Union Pacific Railroad to Central Kansas Conservancy (nonprofit corporation)

Quitclaim deed for Union Pacific Railroad right-of-way located in various sections and townships, including the western half of the eastern half of Section 32, T18S, R3W, except for three 100-ft-wide tracts of land in the northwest quarter of the Southeast Quarter of Section 32, which had been deeded to the railroad in earlier documents (and which were subsequently transferred by the railroad to the co-op). These include the $8 / 30 / 1899$ document and the 6/3/1904 document listed above, as well as the deed dated 12/19/1899 (not on file) referred to in the $6 / 3 / 1904$ document.

12/9/1997 Howse to Ledell Joint tenancy warranty deed for southern half of the Northeast Quarter of Section 32, T18S, R3W, except for railroad right-of-way and the parcel (100 ft by $379.5 \mathrm{ft}$ ) described in 11/29/1899 document.

Quit claim deed for the northern half of the Northeast Quarter of Section 32, T18S, R3W, except for the railroad right-of-way and the parcel $(499.7 \mathrm{ft}$ by $438 \mathrm{ft}$ by $441.6 \mathrm{ft}$ by $387 \mathrm{ft}$ ) described in 2/5/1973 document.

12/9/1997 Ledell to Ledel

Right-of-way easement for land in the Northeast Quarter of Section 32, T18S, R3W, located west of the

Houchen to Rural Wate District No. 6 railroad (about 4.44 acres), described in 2/5/1973 document.

9/30/2002 Union Pacific Railroad to Perpetual easement deed for existing easement in the Northeast Quarter and Southeast Quarter of AT\&T Corporation Section 32, T18S, R3W. (The deed transfers parcels in numerous sections and townships.) 
TABLE B.1 (Cont.)

\begin{tabular}{ll}
\hline Date $\quad$ Item $\quad$ Property Description \\
\hline
\end{tabular}

Property Transfer Records (cont.)

10/6/2003 Foster to Foster Farms, a General warranty deed conveying nine parcels of land in McPherson County. Parcel 4 is the Northwest

Kansas partnership Quarter of Section 32, T18S, R3W, except for a tract of land in the northwest corner of the northwest

\section{quarter described in detail. (The former CCC/USDA facility was within this property.)}

Additional Documentation

$\begin{array}{lll}1884 & \text { Plat map } & \text { Plat map of Township } 18 \text { South, Range 3 West. } \\ 1903 & \text { Plat map } & \text { Plat map of Township } 18 \text { South, Range 3 West. } \\ 1921 & \text { Plat map } & \text { Plat map of Township } 18 \text { South, Range 3 West. } \\ 1969 & \text { Plat map } & \text { Plat map of Township 18 South, Range 3 West. } \\ 8 / 26 / 2005 & \begin{array}{l}\text { Current ownership } \\ \text { information }\end{array} & \begin{array}{l}\text { Current ownership information from the McPherson County Mapping Department, including parcel } \\ \text { identification report for Section 32, Township 18 South, Range 3 West. }\end{array}\end{array}$

a Reproduced documents follow table. 


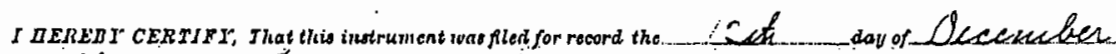
180.8 , at 320

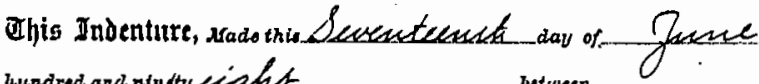

Doputy. If teriste Megister of Dosdh. Mundrad ani ninfty sight

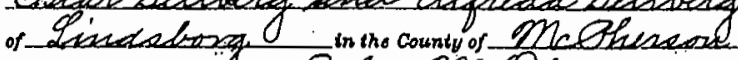
particats the prot part, and Gohes on Oleow wacrife

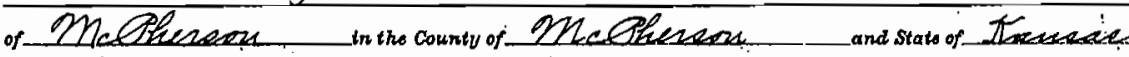
partienfofits cesond part:

Rastry (sd) TITSESSETE, Tikat the ogld partieed in tho your of our. Lord one thousand elfith

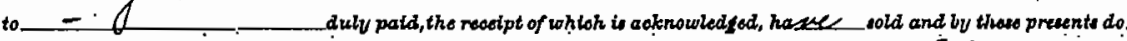
Grant, Bargain and Sell, Convey and Conftron, to the satd parteces of the reoond part, Steine of the firot part in conoliteration of the sumb of

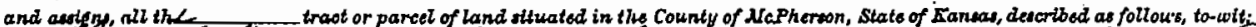

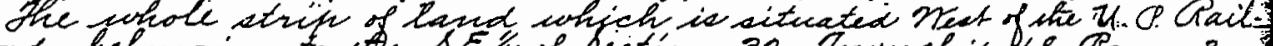

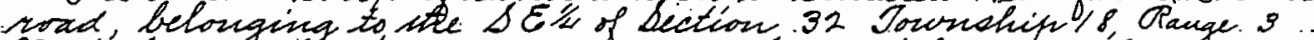

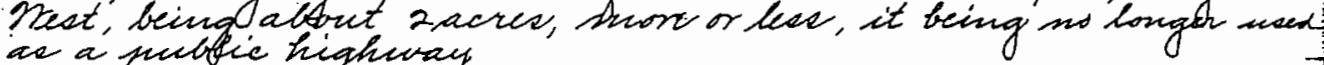
as a putelic higheray

with the appurtenaross, and all the ertate, tille and interest of eald part of the firet part therein.

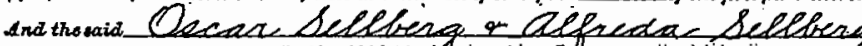

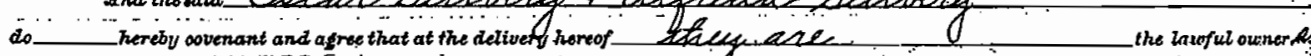

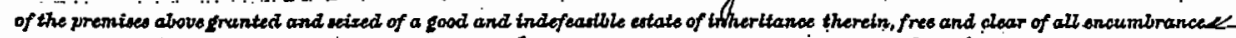

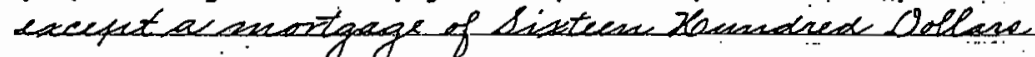

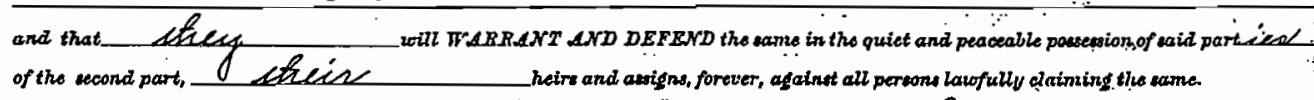

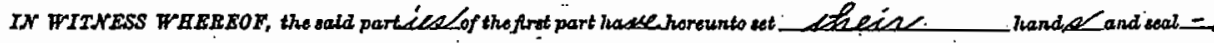
the day and year above written.

Sisned, Seated and delivered in presence of

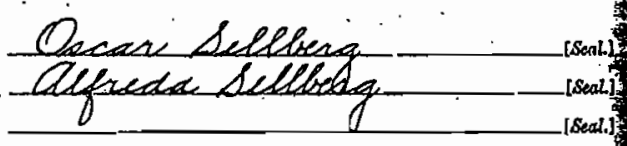

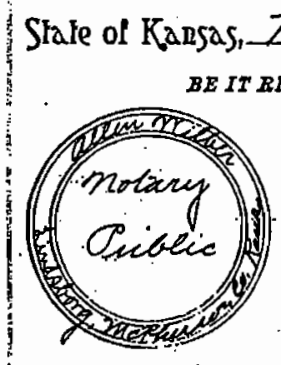

(Ut, $A$ Dasumentery Q. $\%, 12-8 \rightarrow 8$
Counly, ss. :

$8 "$ hurdred and ninetysight whthin and for eald Count and shate perwonally came,

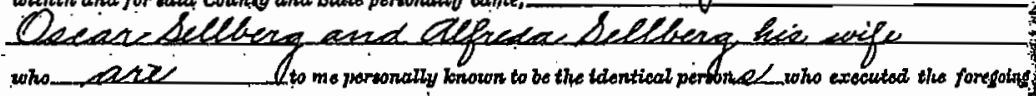
instrantent and duly asknowled ded tho essoution of the santes.

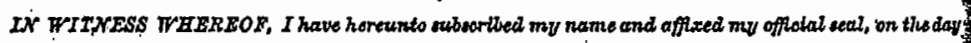
and year last above writter.

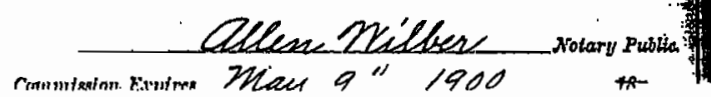




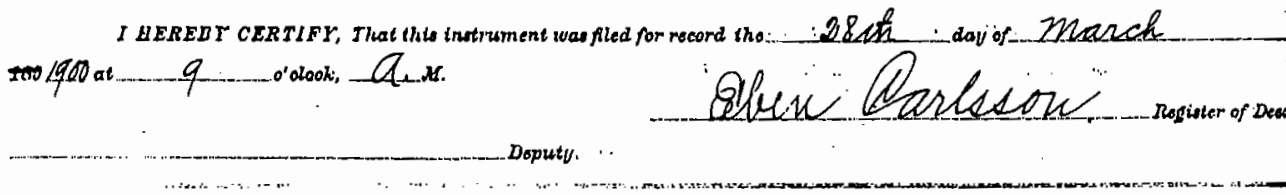

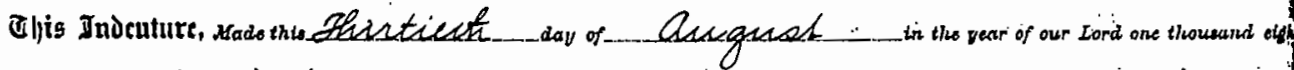
huerdred and ninftuescisse.

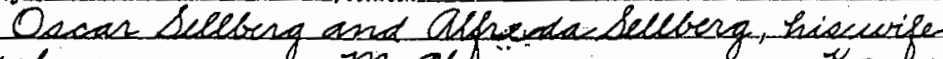

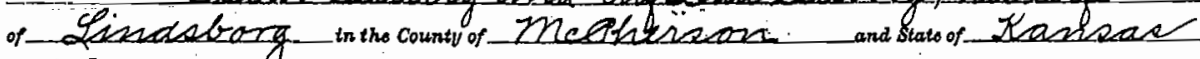
particeff the firu part, and

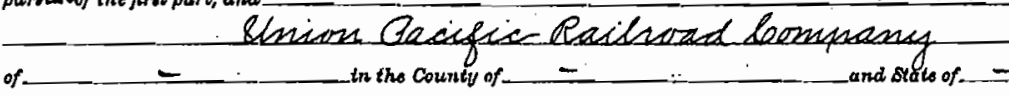

party. of tlus eccond part:

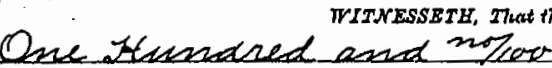

of the firot part in consideration of the sum.

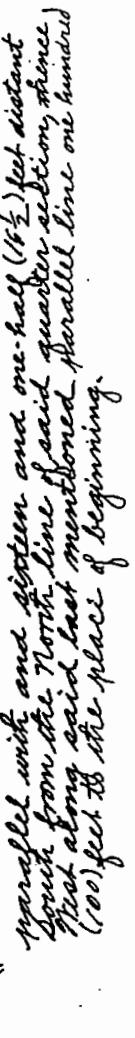

to. thenes

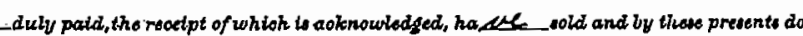

Grant, Bargain and Sell, Convoy and Confirm, to the eaid party of the scoond part,

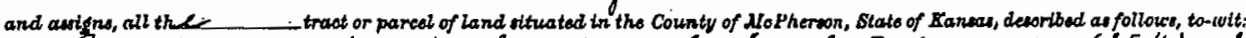
Q strip and trach of land in the South East quarten $(8.6 . \%)$ of becti

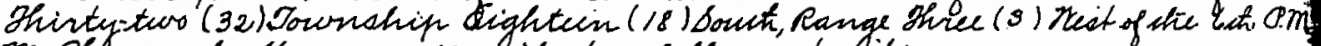
Moblusoon 60. Kaneare, alechibed as followe, tosoit:

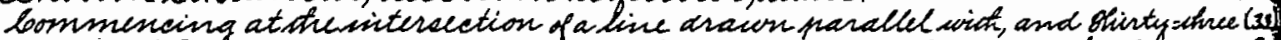

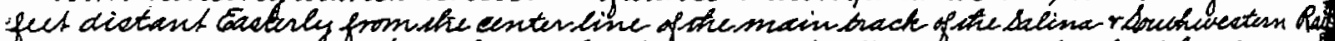
way chin meacused at vight angles to said center line of main tract, with a line dath

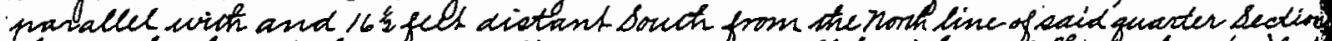

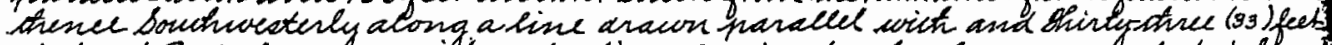

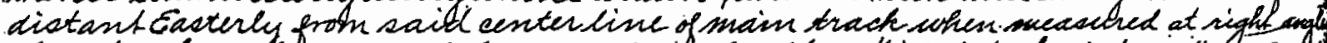

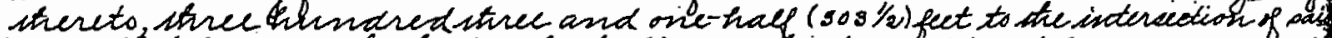
parallel line with the 7 oriherly line of ste tract of land known and descris

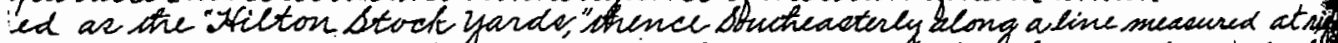
angles from caid centerlinte of mainstract, One thendred 100 feet stence nonteasterly alo:

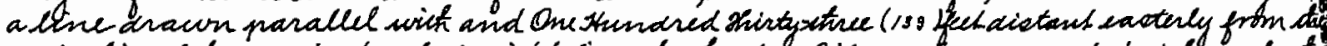

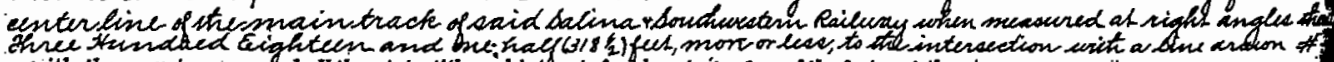
with the appurtenarices, ind all the estate, thise and interest of exid partices - of tho first pert therein.

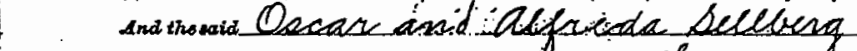

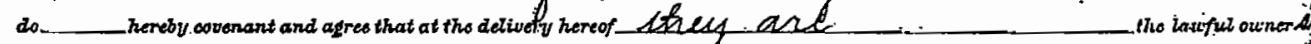

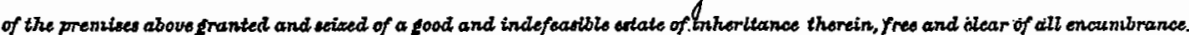

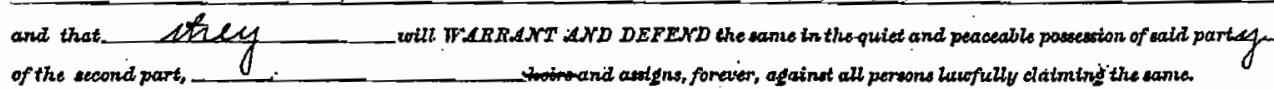

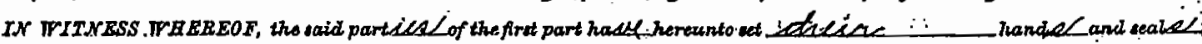

Sirned, Sealed and delivered in presence of

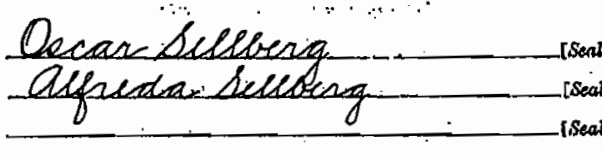

Srate of Kansas, MeQheasen County, ss.

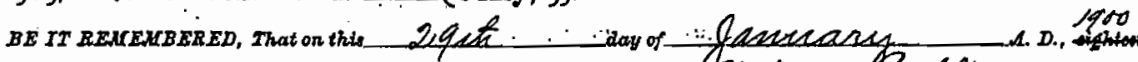

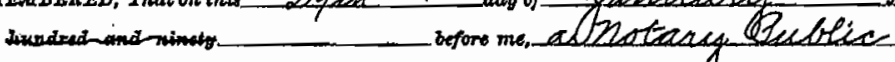

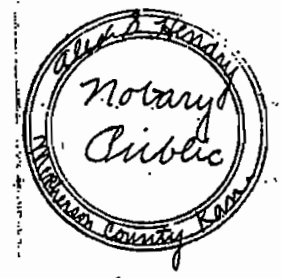

within and for said Corrity ard State perzonally cante,

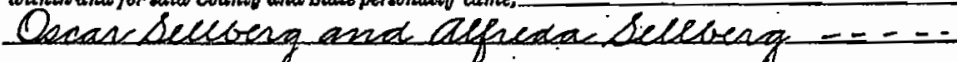

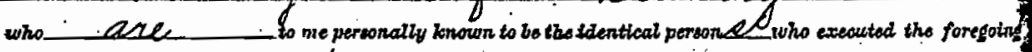
instrument and duly aoknowledsed the avosution of the wante.

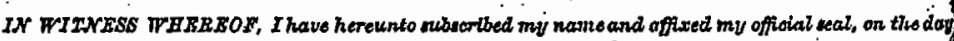
and yoar last above writtem. 


\section{DEED RECORD. $10056,18 \% 2$}

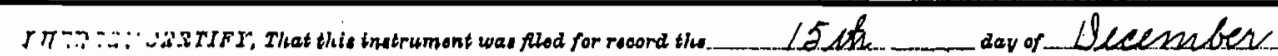

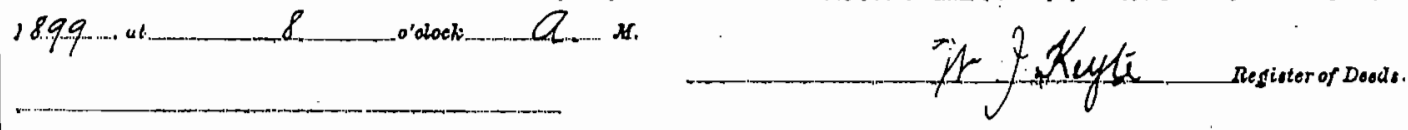

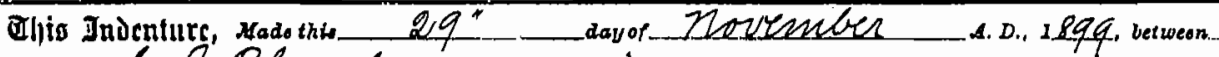
6 g elombeng, a videnerer

of MoRhenson County, in the stato of Transase

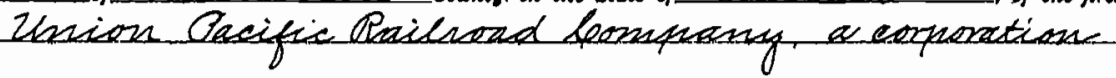

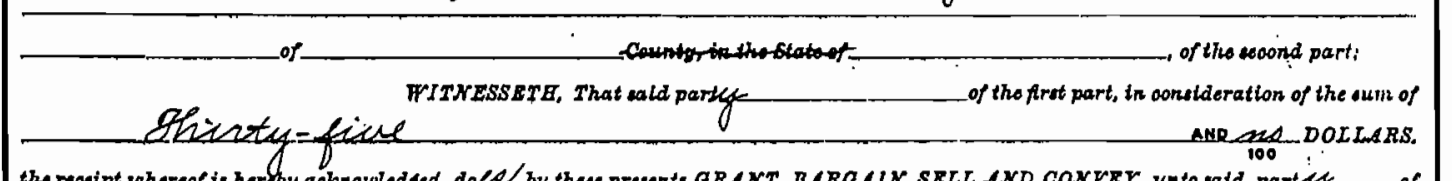

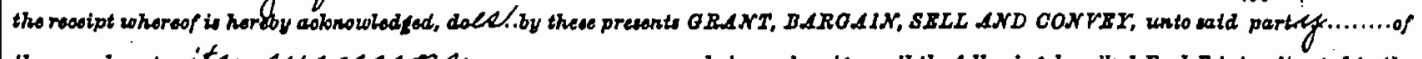

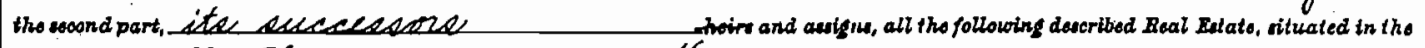

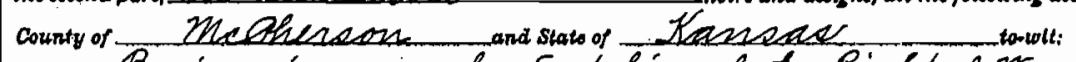
Bouth Meginning on the East hine of the Right of May of the Salina and

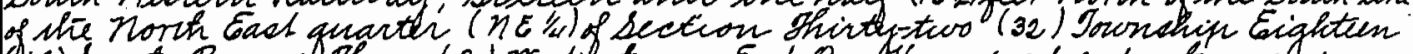
$(18$ ) Dowt, Range Ihree (3) Mest, strence East One Surndred feet, thence in a Northerly dirketion parallel to Right of Nay three hindred seventy-

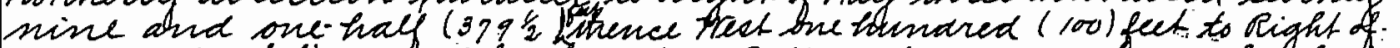

rray, of said Balina and Bouct Mectern Railury lompany, Thence Bouth along

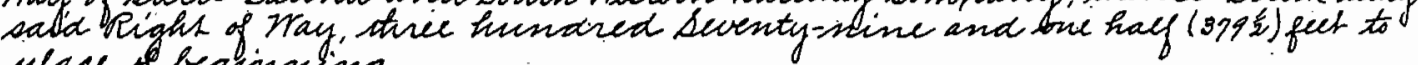
pelace of beginning.

TO. HATE AND TO BOLD TEE SAME. TOgother with all and singular the tonements, hereditament's and appurtenances thereunto belonging or in anywise appertaining forever:

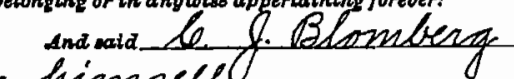

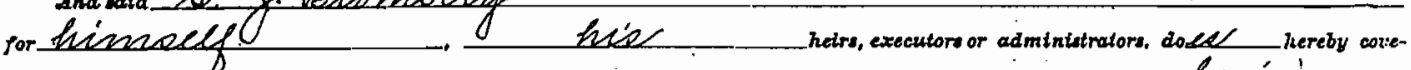
nant, promise and agree, to and with satd party _of the second part, that at the delivery of. these presents he Let zaufully seired, in hise own rlght, of an absolute and ind efeasiblo estate of inhertlance, in fee simpte, of and in all and singular the ajove granted and desoribed premitees, whth the appurtenances; that the eame are free, olear, discharged and uninoumbered of and from all former and other grants, tiltes, oharges, solates, judigments, tavs, astesments and incumbrances, of what nature or kind eoeveri

and that he -will Warrant and Forever Defend the canne unto said party

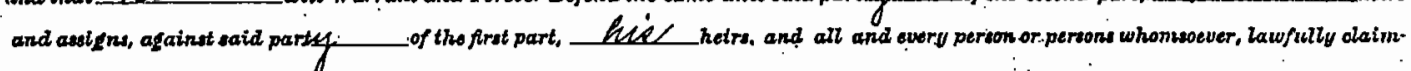
ing or to clatm the eanze.

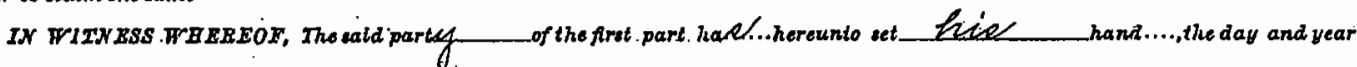
Arat above written.

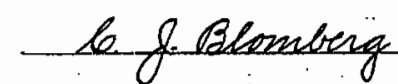

Statc of thansag, fttcpljerson đountu, sg.

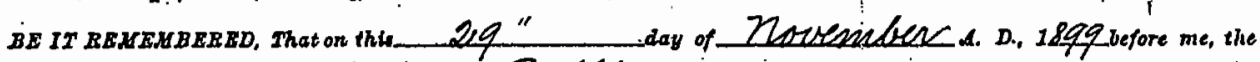

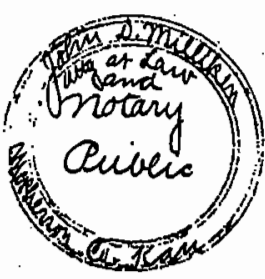

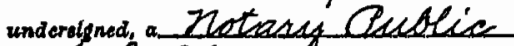
lo folombeng, a vidower In and for tho county and state aforcsald, came who les_ personally inown to mo to be the

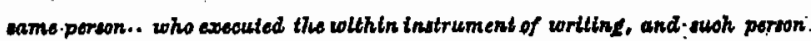
duly aoknowi caded the arcoution of the eame.

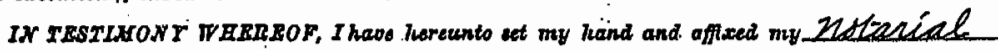
Seal the day and year last above worliten. 


\section{DEED RECORD.}

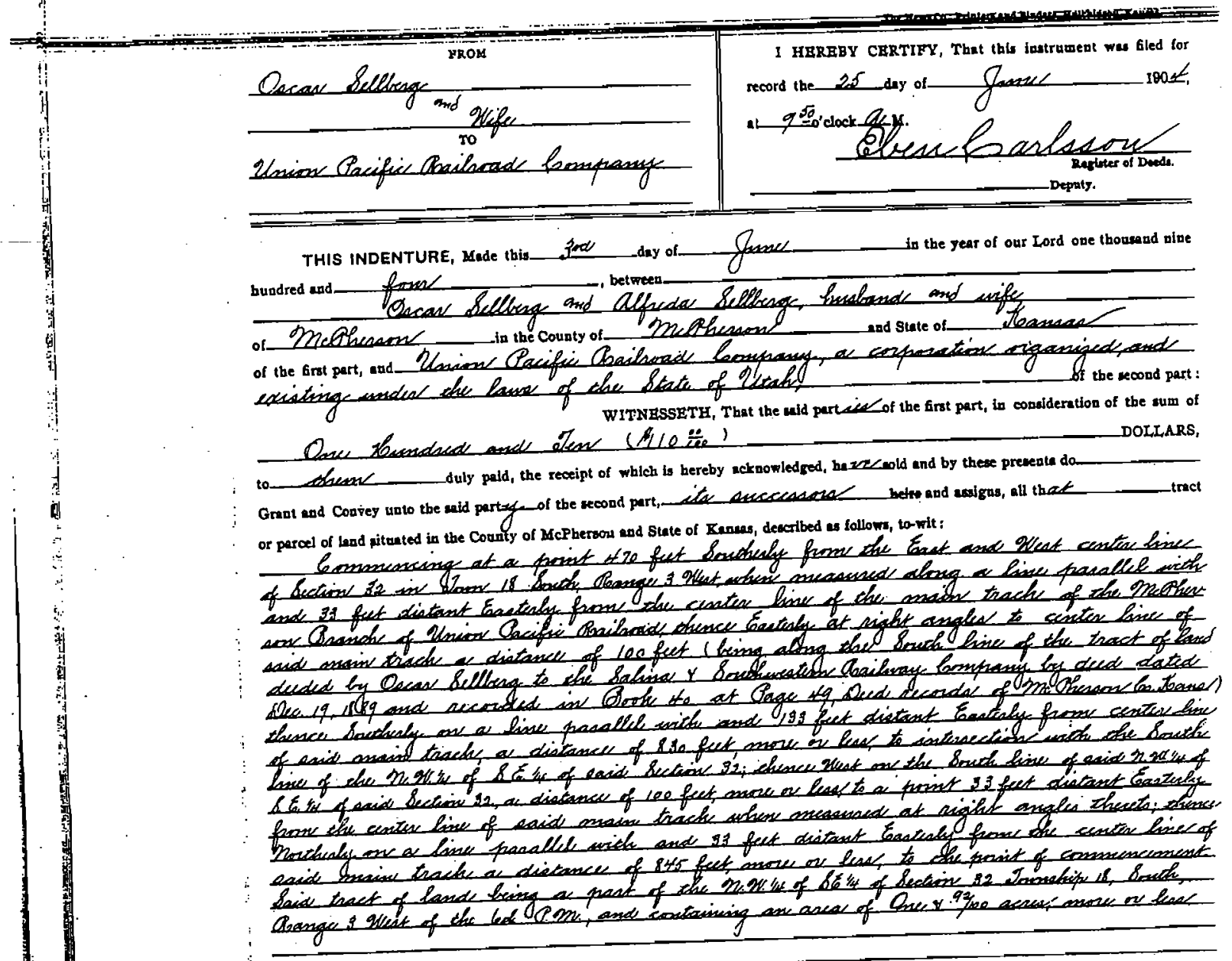

with all the appurtenances, and all the estate, title and intereat of the sald partices of the first part therein.

with all the appurtenances, and all the estate, tidle and intereat of the sald partciator the first part

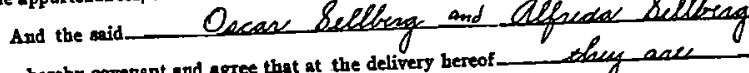

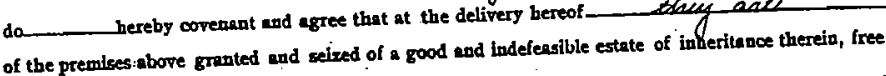

will WARRANT AND DEFEND the ande in the quiet and peaceable poasession of the seid party

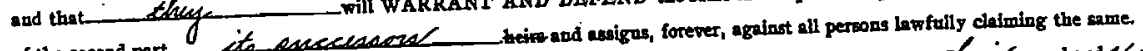

of the second part,

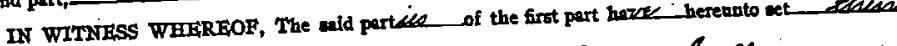

the dey and year first above written.

Signed, sealed and delivered in presence of

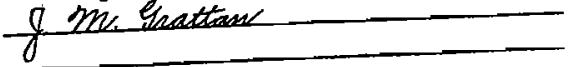

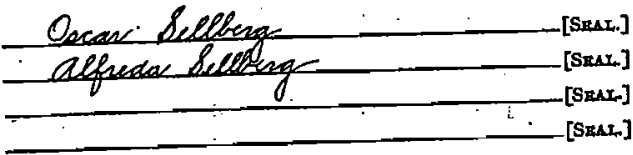

STATE OF KANSAS, COUNTY OF MCPHER8ON, 85.

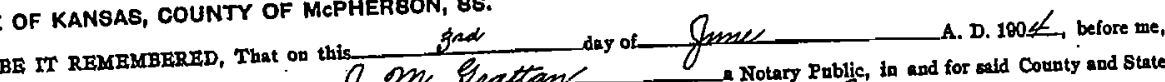

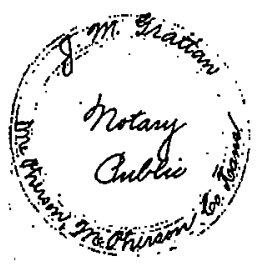

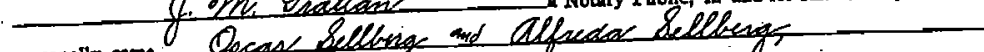

of who executed the toxegolng instriment und duly ac

to me persoudly known to be the

knowiedged the exectution of the seme.

IN WITXKESS WHEREOF,

day and year last above written.

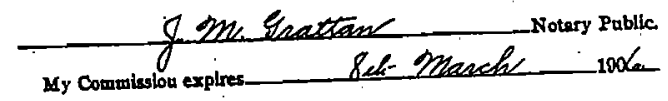


DEeD KELUKL IVO. IUY

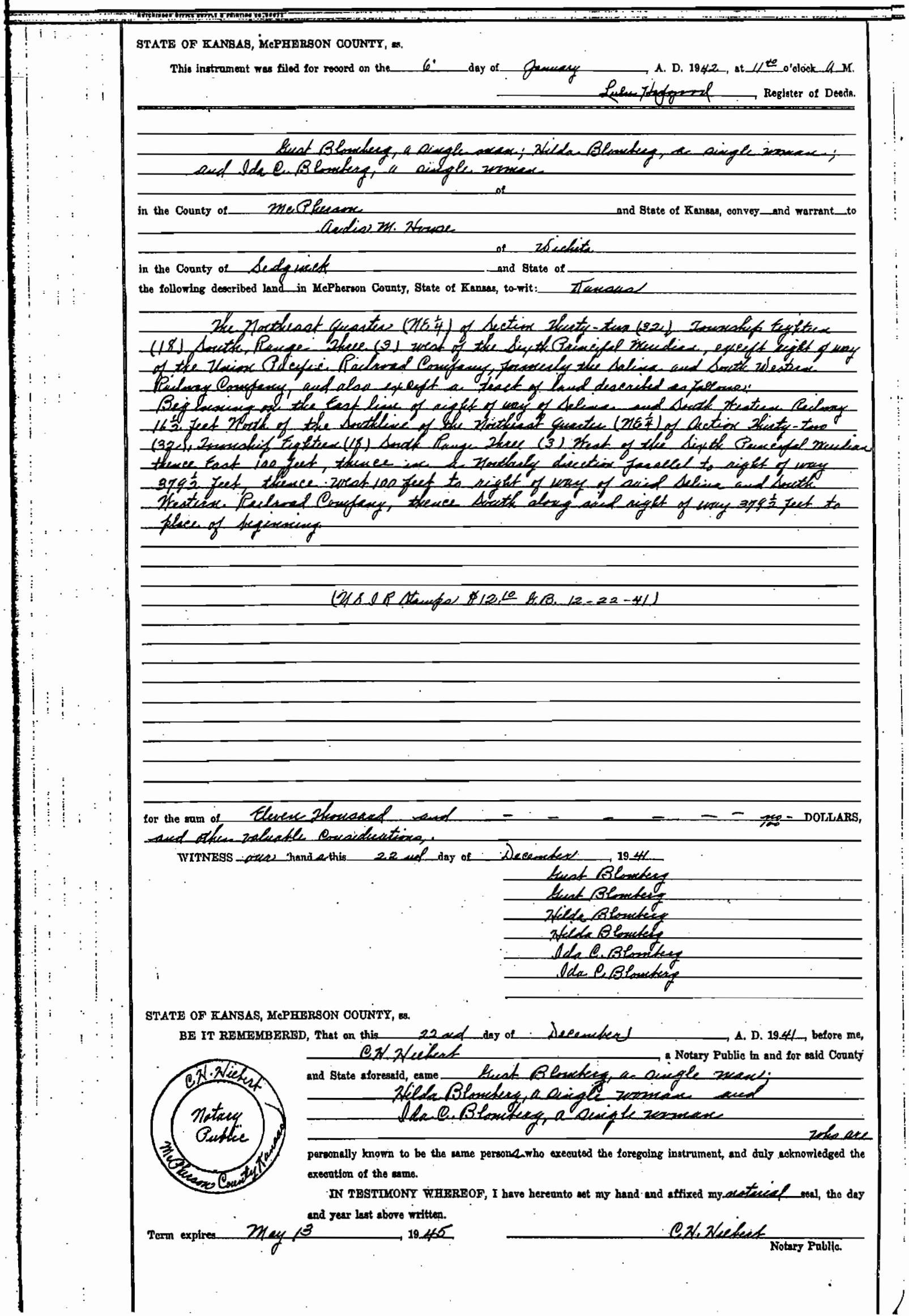




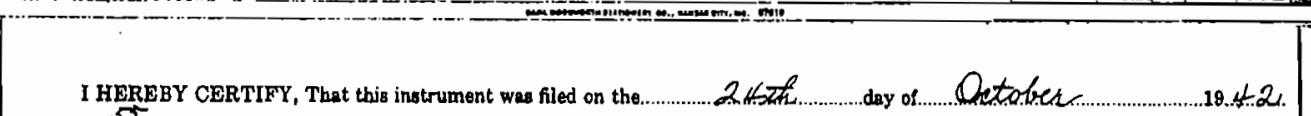
at $10 \stackrel{5}{5}$.

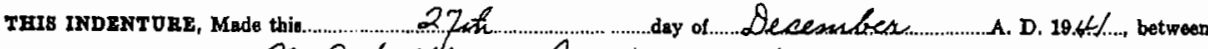

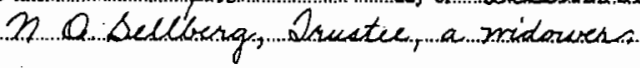

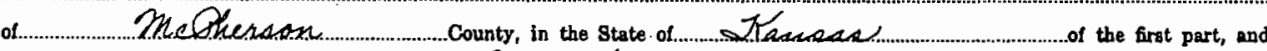
Oeran bellowige

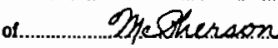

County, in the State of J Texueas

of the recond part WITNESSETH, That said partyj....of the first part, in consideration of the num of One Y .....os the first part in $\rightarrow$ DOLLARS, the receipt of which is hereby ecknowledged, doces by these presents REMISE, RELEABE and QUIT CLAIM unto usid partixy . of the eccond part, Lhes ......heirs and aesigus, all the following-described real eatate, situated in the County of MaPhernon, und State of Kansas, to-wit:

Ale of the Sonstheat Quarter (SE/4) of Section

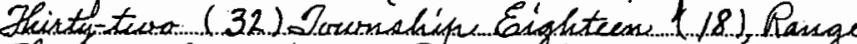

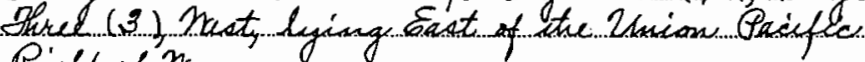
Right of Yray.

This relingsimehes all wil asd gas Poyralty Pighto thise

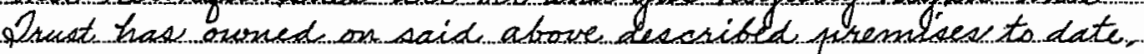

TO HAVE AND TO EOLD THE SAME, TOgether with all and singular the tenemente, hereditaments and appurtenances thereunto belonging or in angwise appertaining forever.

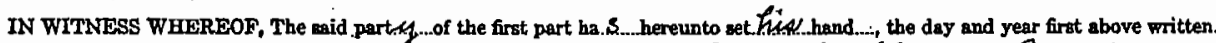

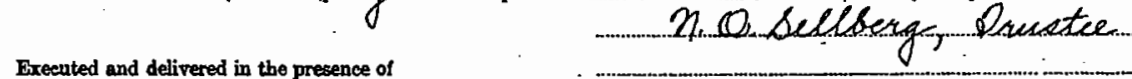

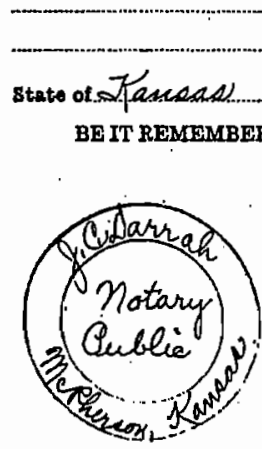

Meqhensans . counts, as.

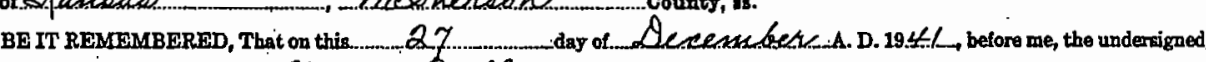
- Notany Sublie a.notin and for the county and

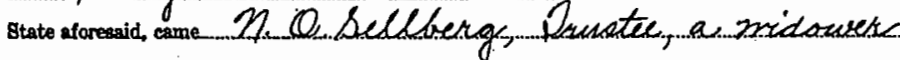

who._._e _._._...perwonally known to me to be the same person.... Who executed the within instrument of writing and wuch person duty acionowiedged the erecution of the game.

IN TESTMONY WHEREOF, I have hereunto set my hend and affixed my. seal, the day and year last above witten.

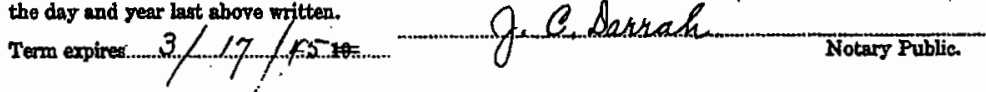




\section{Deed Record No. $114 \quad$ pg $/ 28$

I HERTEY CERTIFX, That this instrument was filed for record the 3 at $10 \mathrm{~T}$ -

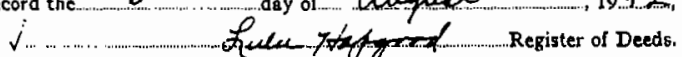

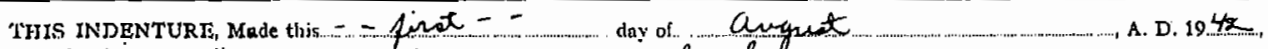

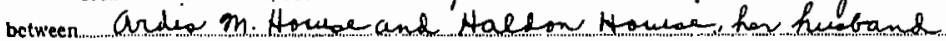

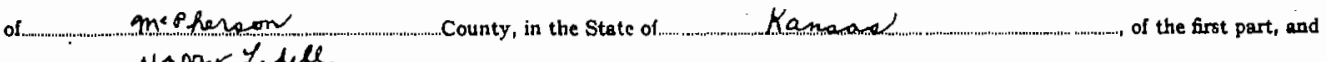
Nomy Lelle County, in the Statc of ........... Kamanare Courty, in the State of ...............mean , of the second part:

of mephonon

WITNESSETH, That said partines of of the first part, in consideration of the sum of $-(n, 500.00)$

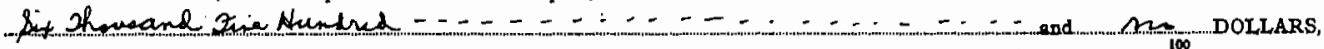
the recespt whereof is hereby acknowledged, do - - ...... by these presents, Grant, Bargain, Sell and Convey unto said part. $y$. part,.......... his . heirs. and assigns, all the following described Real Estate, situated in the County of McPherson, and State of Kansas, to-wit:

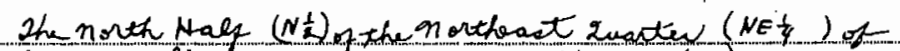

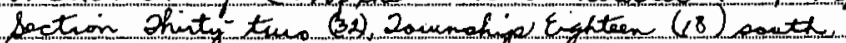

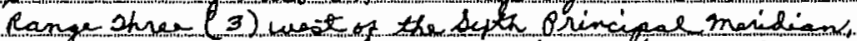

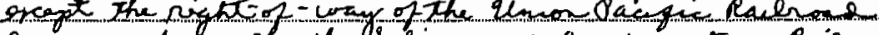

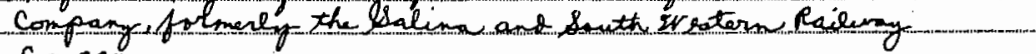
Company

TO HAVE AND TO HOLD THE SAME, Together with all and singular the tenements, hereditaments and appurtesances thereunto belonging or in anywise appertaining, forever.

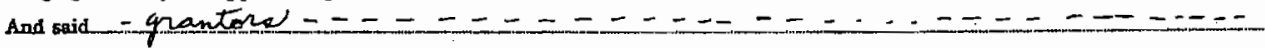

$$
\text { sivertion }
$$
to and with said party. of the second part, that at the delivery of these presents.
seized in
singular the above granted and described premises, rith the appurtenances; that the same are free, clear, discharged and unincumbered of and
from all former and other grants, titles, charges, estates, judgments, taxes, assessments and incumbrances, of what uature or kind soever;
onome. from all former and other grants, tittes,
none.

and that thay will WARRANT AND FOREVER DEFEND the same unto said part $y$ ___ of the second part, flos heirs and assigus, against said partien of the first part,_thein - _ beirs, and all and every person or persons whomsoever, lemfully claiming or to claim the seme.

IN WITNESS WHEREOF, The said partines of the first part hase hereunto set_theis____ hand the the day and year first above written.

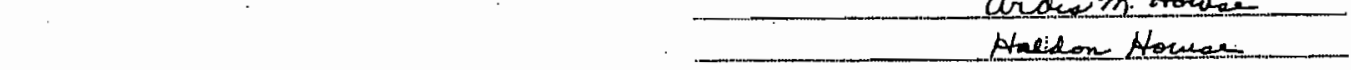 \\ STATE OF KANSAS, MaPHERSON COUNTX, Bs.

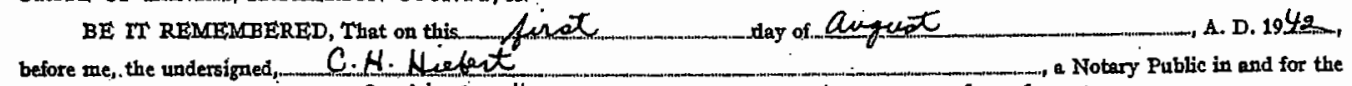

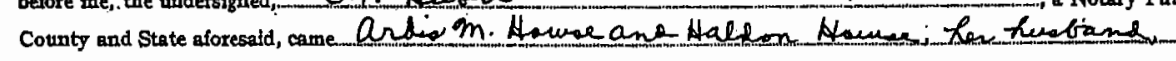

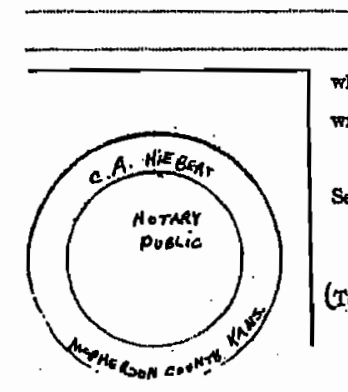

who ane personally known to me to be the same person who exceuted the within instrument of writing, and such persons duly scknowiedged the exeastion of the same.

IN TESTMMONY WHEREOF, I have heresnto bet my hand and affixed my Seal, the day and year last above written. 
11. No merber of or Delegate to Congrees or Resident Conulsaioner, shall be adnitted to any share or part of this lesse or purchase or to any benefit that may arise therefrom, but this provision shall not be construed to extend to this lease or purchase if made with a corporation for its general benefit.

12. The Lessor warrants that he has not employed any person to solicit or secure this leas upon any agreement for a comission, percentege, brokerage, or contingent fee and that no such consideration or payment has been or will be made. Breach of this warzanty shall glve cCC. the right to annul the lease, or, in its discretion, to deduct from the rentel or purchase price the anount of such commission, percentege, brokerage or contingent fees. This warranty shall not apply to comiseione pajable by the Lessor if the lease is secured or made through a bona-fide agent maintained by the Lesso for the purpose or leasing or selling his property.

(SEAL) Frark E. Dyck LESSOR

(SEAL) - LESSOR

CONODITY GREDIT CORPORATION, LESSES

(SEAL)

LESSOR.

BI Jacob A. Hedel

(To be reproduced in ASC STate Office)

Chairman, $\mathrm{H}_{\mathrm{c}} \mathrm{Ph}$ herson County ASC Committer

SIATE OF KANSAS, MCPHERSON CONNTY,

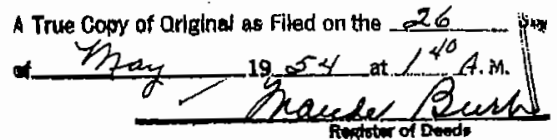

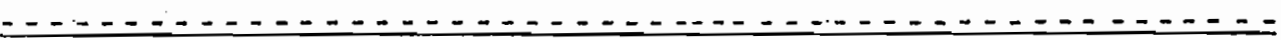

CL Form -58

$(4-2-54)$

U. S. DEPARTMENT OF AGRICVLTURE

AGRICULTURE STABITIZATTON AND CORSERVATION

GOMODITY CREDIT CORPORATTON

LEASE OF PROPERTY

THIS LEASE, made and entered into this 1st day of May, 1954, by and between Keith Peterson, by Arvid L. Peterson, of McPherson, Kansas Iessor, and Comrodity Gredit Corporation, Le ssee./ Agent

WITESSETH THAT:

1. The Iessor leases to the Lessee, and the Iessee hereby leases from the Lessor, upon the terms and conditions hereinafter stated, the foll owing described real estate. (hereinafter called "property") situated in the County of McPherson and state of Kansas:

Beginning in the southesst corner of the SE af the Nur 32-18-3, from the center of Public Highray 510 feet north; thence 128 feet west; thence 510 feet south; thence 128 feet east to the point of beginning, containing 1.5 acres, more or less.

2. The term of the lease shall be for a period of 5 jears, commencingthe lst day of May, 1954, and ending the lst day of May, 1959, wth the right of the Lessee, during such term or any extension thereor, to terminate said lease, and liability $f$ or any further rent, on the lst day of ulay of any year, by giving 30 days' previous notice in writing to the tessor.

3. As rent for said property, the Lessee shall pay the Lessor ginty Dollars $(\$ 60.00)$ per year, such rent to be payable in advance, but to be apportionable in the event the lease is terminated as provlded in paragraph 2 hereaf:

4. The Leser warrants that he 15 the owner of the property, has the right to give the Iesses posiession under this lease, and will, so long as thie lesse remains in effeot, warrant and defend the Lessee's possession agalnst any and all persons whorasoever.

5. The Ieesee shall have the right, during this lease, to erect storage strupotures, or facilities, make alterations, ingtall scales, fenoes, or slgns, in or upon the premises hereby leased and, at the dxplration of eaid lease or any renewal or extension thereof or at any time this lease $1 \mathrm{~s}$ in effect, may renove said storage structures, facilities, ocales, fences, or signs or any part thereof whether or not such structures, facilities, scales, fences or signs have become legally a fixture.

1.

5. The Lesses shall not aselgn this leaselithout the witten consent of the Lessor. The Lesee, may howerer, sublet the structures on the premises leased hereunder, or any one or niore of themior the term of the lease or ap part thereof upon such terms and oonditions as ceasee may wish to so sublet.

7. The Les8ee, if required by the Les8or, shall upon the explration of this leage, or renewal thereof, restore the premises to the same condition as that existing at the time of entering upon the same under thie lease, reasonable and ordinary wear and tear and dameges by the olements or by ofrcumstanoes over which the tessee has notcontrol excepted. Frovided, horever, That if the Lessor fequires such restoration, the Lessor sholl give written notice thereof to the Iessee 50 days before the termination of the leare.

of Forrs -58 (Reverse)

$(+-2-54)$

\section{LEASE OF PROPERTY}

9. As a consideration of this lease and for the further oonsideration of one dollar, the reoelpt of which 18 hereby acknowledged, the Leseor grante and gives the Lessee the option, at ans time wille this lease is in effect, to purchase said property from the Iessor, his heirs, executors, adninistrators, and aseigns, for the oum of Dollare (f) ). In the erent the Fessee shal exercise this option to purohase said property, the lesscragrees to furnish at his own expepse an abatract of titile, oertifiaate of title, or other evidence of titile satiofactory to cce and to exceoute a good and oufficient warraity deed convering fee stmple title to aald property free and olear af all taxes, liens, or enoumberanoes except for the follouring, snd no others. 
10. In the event any increased tex agsessment is made against the Lessor or the property by virtue of the erection of storage structures and facilities thereon by the Lessee, the Lessor agreas to cooperate fully in any contest of such increased sssesement which the Lessee feels should be msds. The Lesser agrees that the rental hereunder shall be adjusted upward by the anount of any such increas ed tax asssssment which the Lessor and Lesses mutually agree to be proper or which is determined to be legally valid in court proceedings.

11. No nember of or Delogate to Congress or Resident Commissioner, shall be admitted to any share or part of this lease or purchase or to any benefit that may arise therefrom, but this pro-

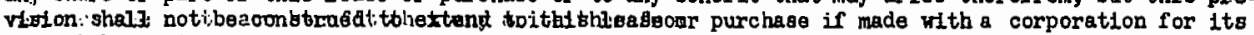
general benefit.

12. The Lessor warrants that ho has not employed any person to solicit or secure this lease upon an agreement for a comission, percentage, brokerage, or contingent fee and that no such consideration or payment has been or will be made. Breach of this warranty shall give CCC the right to anmul the lease, or, in its discretion, to deduct from the rental or purchase price the amount of such commission, percentage, brokerege or contingent fees. This warrenty shall not pply to comnissions payt able by the Lessor fierthe lease is secured or made through a bona-fide agent maintained by the Lessor for the purpose of leasing ar selling his property.

(SEAL) kelth Petergion, by Arvid L. Peterson Agt.

(SEAL )

LESSOR
COMYDITY CREDIT CORPORATION, LESSEE

BY Jacob A. Wedel

Ghairman McPherson County ASC Coumittee

( To be reproduced in ASC State office)

INIE OF KANSAS, MCPHERSON CULW TY

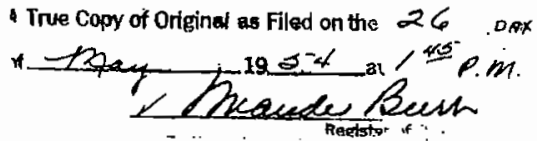

ASSTONGENT

KNOW ALL MEN BT THESE PRESFNTS:

That the undersigned, MUSGROVE PETROLEJ CORPORTAION, INC., hereinafter called Assignor, for and In consideration of the sum of One Dollar $(\$ 1,00)$ and other good and valuabie coneiderations In hand paid, the receipt and sufficiency of which is hereby acknowledged, does hereby sall, assign, transfer. and set over unto MATIONAL COOPERATIVE REFINERT ASSOGIATION, anciunditided one-half (1/2) interest in and to each and all of the oil and gas mining lesses tnsofar as said leases cover the following described lands in MoPherson County, State of Ransas:

Oil and Gas Lease dated Februsary 16, 1954, by and between J. A. Morine and Alma J. Morine, his wife, lessors, in favor of P. C. Musprore, lossee, recorded in Book 95 Miac, Page 543 of the records of McPherson County, Kanseis, described as

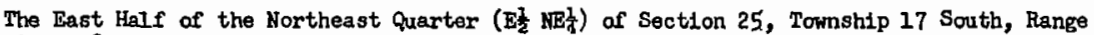
2 West, containing 80 acres, more or less,

OIl and Gas Lease dated February 13, 1954, by and between Esther H. Hedstron, a oingle woman, lessor, in fevor of P. C. Kusgrove, lessee, recorded in Book 96 Misc., page 5 li2 of the records of McPherson County, Kansas, described as 160 acres, more or less,

The Southrest Quarter (Sw $\frac{1}{4}$ ) of Section 25, Tormship 17 South, Range 2 West, containing

Oil and Ges Lease dated Yebruary 13, 1954, by and between Allen I. Nelson, Selma Nelson, Hilma S. Helson, all oingle, lessors, in favor of P. C. Yusgrove, lessed, recorded in Book 96 Misc., page 547 of the records of JicPherion County, Kanses, described as

160 acres, more or less.

The Southeast Quarter $\left(S S_{\frac{1}{4}}\right)$ of Section 25, Township 17 South, Range 2 West, containing

together with the rights incident thereto and the personal property thereon, appurtenant thereto, or used or obtained in connection therentith.

The leases herein assigned are subject to an orerriding royalty intersat of one-sixteenth of seven-elghths of all oil, gas and casinghead gas produeed, eaved and marketed from the above doscribed land under the provisions of the oforesaid leases, or any extensions, or renewel thereof, as an overriding royalty, free and clear of any cost and expense of the development and operation thereof, excepting taxes a pplicable to said interest and the production thereirom. The interest herein assigned is eubject to and shall bear its proportlonate part of the aforementioned overriding ropralty interest. This assignment is made without warrenty of title, either express or Implied.

Executed, this 17th day of May, 1954. 


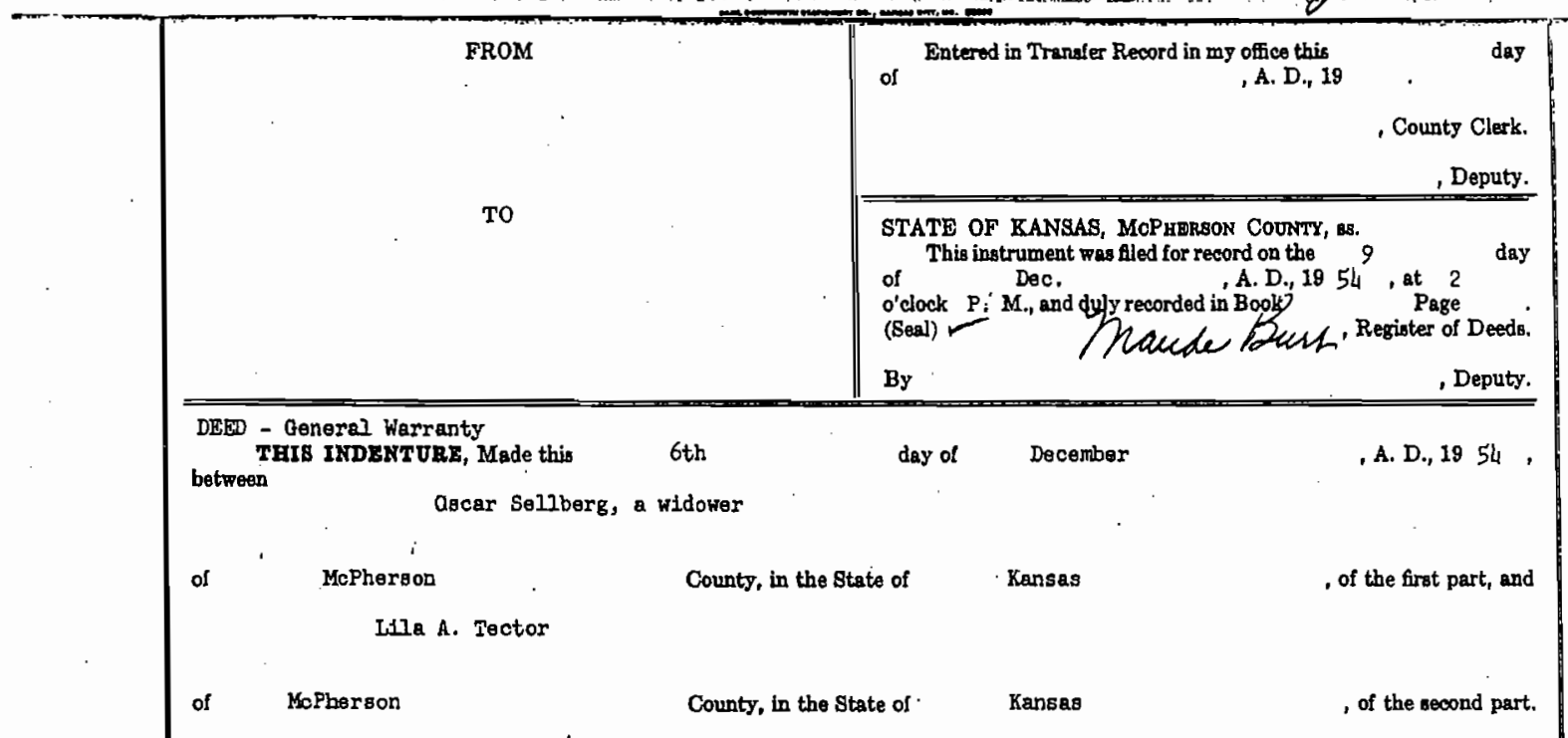

WITNESSETH, That gaid party of the first part, in consideration of the sum of

One Dolir and Iove and Affact1

the receipt whereof is hereby ackmowledged, do 86 by these presenta grant, bargain, sell and convey unto said part y of the second part,

The Southenst Quarter ( $\mathrm{SE}^{2}$ ) lying East of the Dnion Pacific Rall Road

right-of-ikgy of Section Thirtytwo (32) Township Eighteen (16) Range

three (3) West of the 6th P.M. conteining 150 acres more or less

TO BAVE AND TO HOLD THE SAME, Together with all and singular the tenements, hereditaments and appurtenances thereunto belonging or in anywise appertaining, forever.

And said Gronter for htmself,

his heirs, exectors or administrators, do es hereby covenant, promise and agree, to andiFith said part $J$ of the gecond

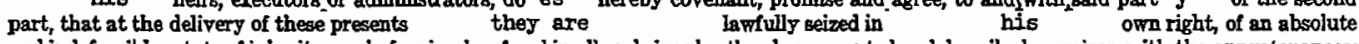
and indefeasible estate of inheritance, in fee simple, of and in all and singular the above-granted and described premises, with the appurtenances; that the same are free, clear, discharged and unincumbered of and from all former and other grants, titles, charges, estates, judgments, taxes, assessments and incumbrances of what nature or kind soever: except Iife Estate reserved by Granter

and that he Fill WARRANT AND FOREVER DEFEND the eame unto said part $y \quad$ of the second part her

i heirs and assigns, against aid part $\mathrm{J}$ of the first part, his heirs, and all and every person or persons, whomsoever, lawhully i claiming or to claim the same. iabove written.

the and all and every person or persons, whomsoever, lawfully

Oscar Șellberg

STATE OF KANSAS, McPherson 6 CoNTYY; ss.
BG.ITREMEMBERED, That on this 6th $\begin{gathered}\text { COONTY; 8s. } \\ \text { day of Deceriber }\end{gathered}$ a Notary Publig Itrapd for the County and State aforesaid, came oscar Selliberg, a widower

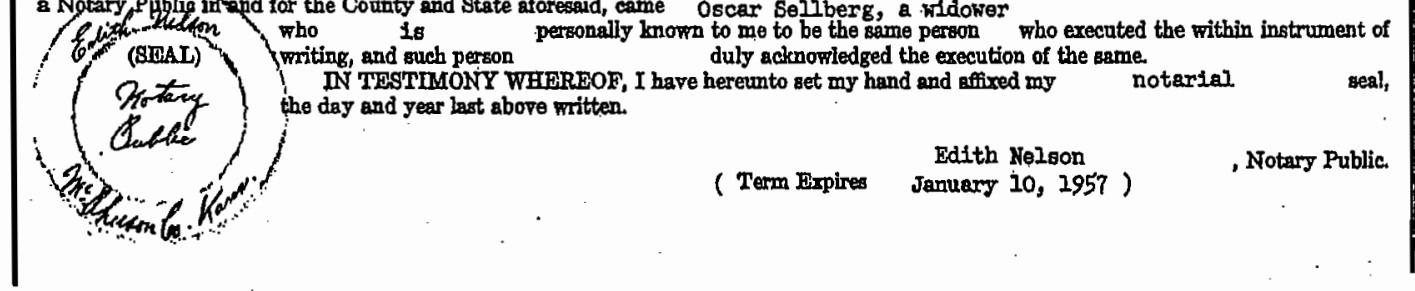


THIS INDENTURE, made this.......29.th. day of.......Jenuary. A. D., 19.52., between Is1a As Tector and Elmer Tector, w1re and husband,

of......Maphenson. County, In the State of.....Kangas of the first part, and McPherson...Angth Co.n. Inc.

of..........McPherson... County, in the State of........KAnsag. of the second part:

WITNESSETH, that sald parthes. of the Arst part, in consideration of the sum of One Doliar (\$1,.00) and other valuable considerat1on Xijox BRTExars the receipt of which ts hereby acknowledged, do...... by these presents Remise, Release and Quit-Claim unto sald part.y.... of the second part,.....h18. heirs and agsigns, all the following described Real Estate situated in the County of McPherson, and State of Kansas, to-wit:

Commencing et the Northwest corner of the following described tract of land, to-w1t: 'The southeast Quarter

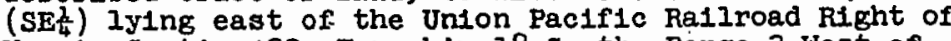
Way in Section 32, Township 18 south, Range 3 West of the 6th F.M., thence in a southerly alrection along sald right of way a distance of 40 rods, thence east 2 rods, thence north 40 rods to a point 2 rods east of the place of beginnting, thence west 2 rods to place of beginning.

The grantor, Ifla A. Tector, for herself, her heirs, executors, and assigns, reserves all o1l, gas and mineral rights under sald tract for a term of 15 years from this date and as long thereafter as o1l and/or gas may be produced from sald premises, Inoluding the exclusive right during said perlod to lease sa1d. land for ofl and/or gas purposes.

TO HAVE AND TO HOLD THE SAME, together with all' and singular the tenemerits, hereditaments and appurtenances thereunto belonging or in anywise appertaining forever:

IN WITNESS WHEREOF, the sald parles. of the flrst part have hereunto set.the1 $x$. hand.s. the day and year first above written.

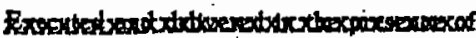
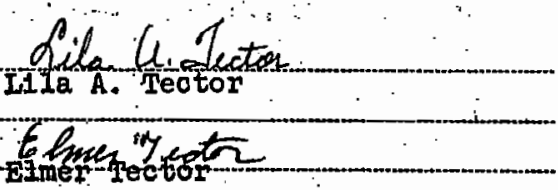


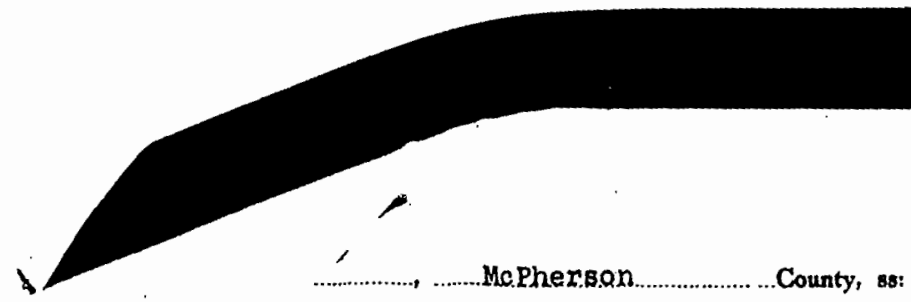

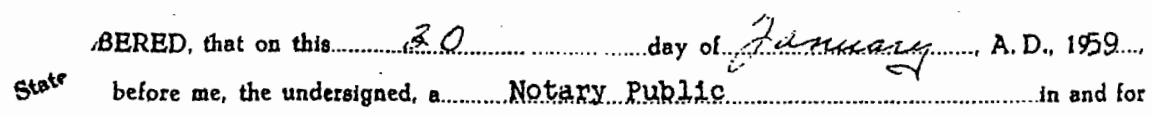

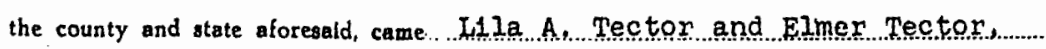
Hsfe and husband,

who.....ene......

personally known to me to be the same person $s$ who executed the within instrument of writing, and such peraon .8 .............duly acknowledged the exceution of the same.

$\because, 0.0 \%$

IN TESTIMONY WHEREOF, I have hereunto get my hand and affixed
in $\therefore$ my notarial seal the day and year last above written.

$\rightarrow b_{n+1}+4$

$\therefore \ldots$

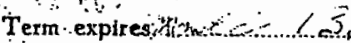

$19.6 \dot{1}$
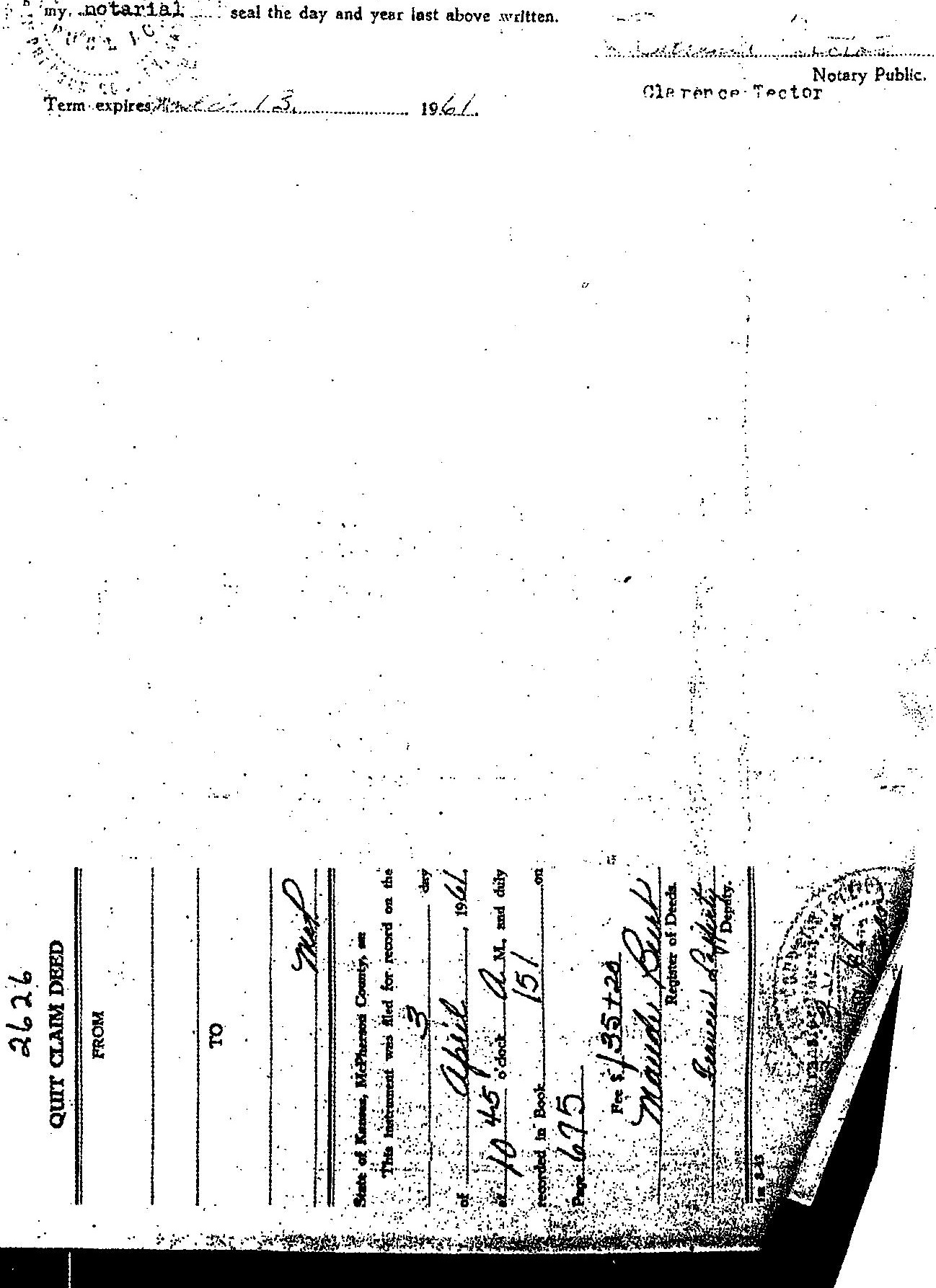


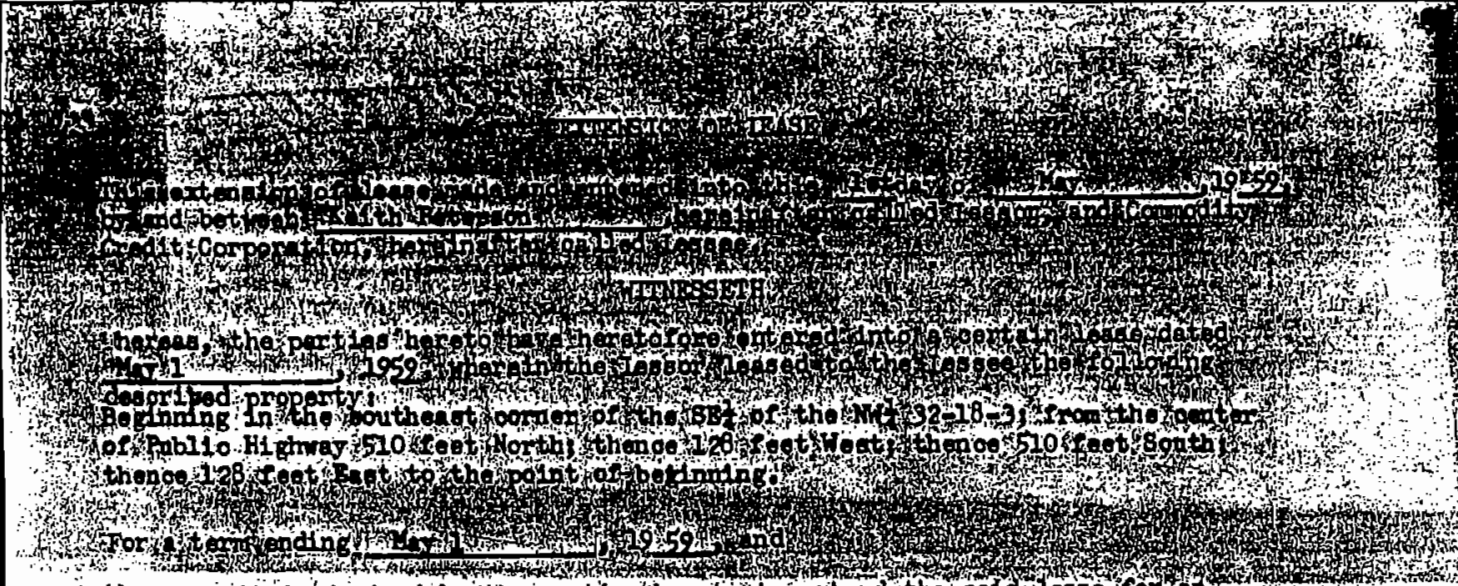

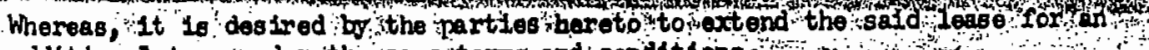

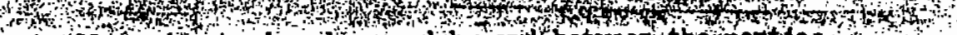

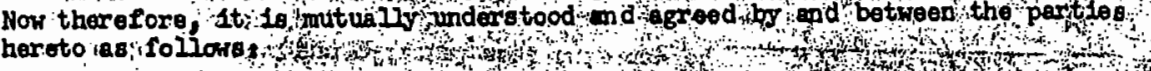

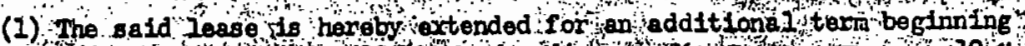
May. 10 isgond anding

$$
\text { (t) }
$$

(2) Iese ore grant and giveto the Ioses the option of further renewa 2 of to

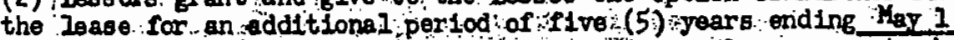

1969 ; under the eame torms and oondit tonenof the Joese as extended herein

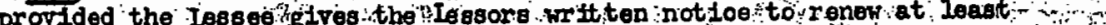
thirty (30) days priorito the time the Ioase jas axtended lould othorw se explre.

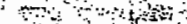

In W1tress. Whereof, the put les hereto bere exeouted this extension on the day firgt apore mattien.

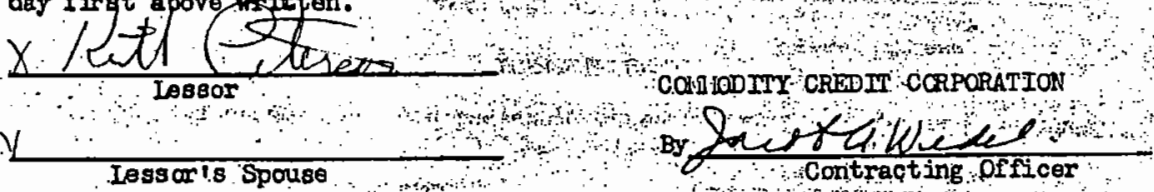

Note: It 1s.neoesery to have the above agreement jecknowiedged and recorded.

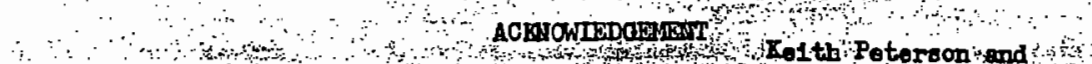 do hereby certify that Jacob. A. Wedel}

I. to.mo

known to be the pers on :(or persons) ano sexouted the ? Forego ing instrument,

personaliy : appeared ibefore me and acknghedged that he (sho or they) exeouted the

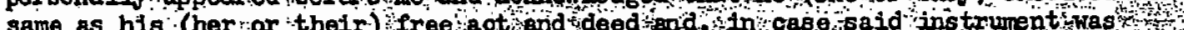

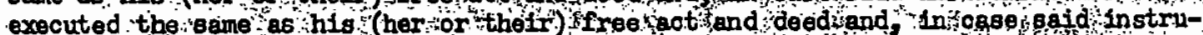

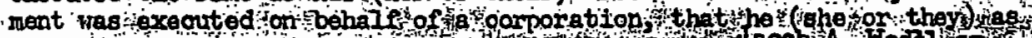

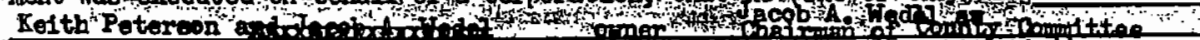

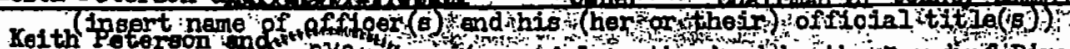

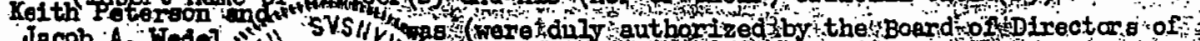

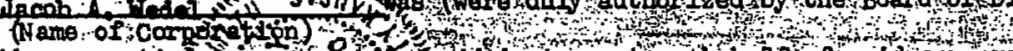

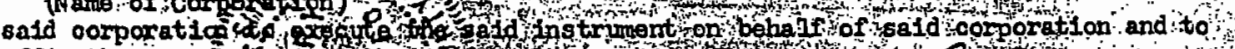

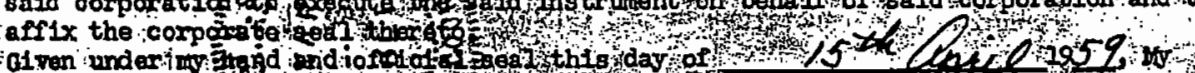

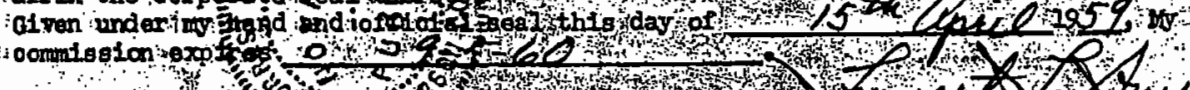
(n)

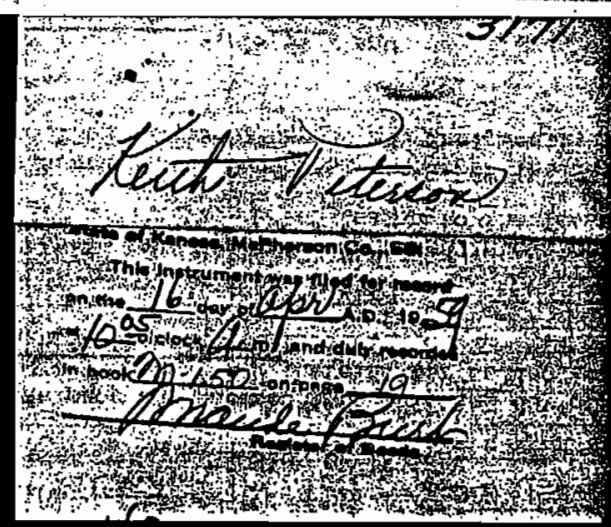


THIS LEASE, made and ontered toto thin

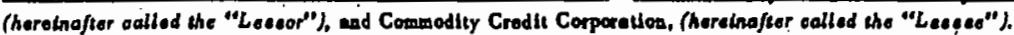
WITKDSSETH TEAT:

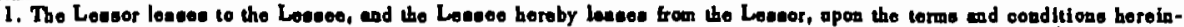

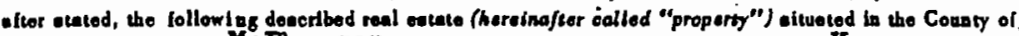

(Enter hare a complete logal deceription of the elte) Kana8s

Beginning in the Southeast corner of the SBh of the NWK 32-18-3; from the Center of Fublic Highway 510 feet North; thenoe 128 feet West; thence 510 feet Bouth; thence 128 feet East to the point of beginning.

containing 1.5 acres, more or lees.

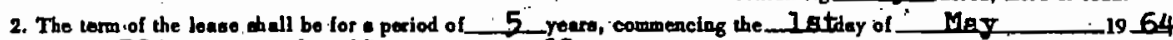

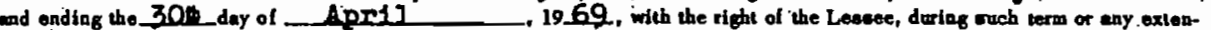
sion thereol, to cerminate axid leace, and lieblitity for any ferther rene, on the latiney of . Mey of any year, by ofving 30 days' previous notice in writing to the Leweor.

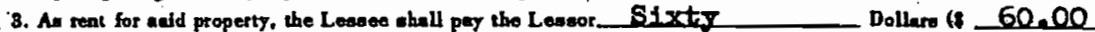
per yoar, wreh reat to be payable In advance, but to bo epportionable in the event the lense lo terminated as provided in paragraph 2 hereof:

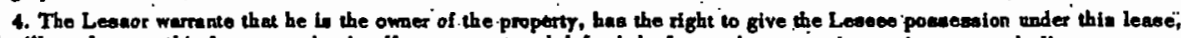

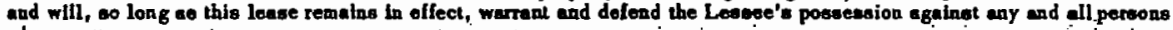
whomoever.

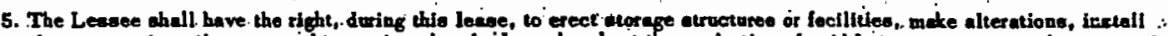

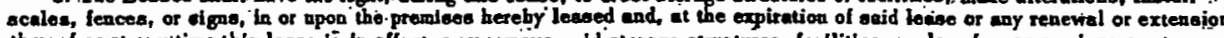
thereol or at eny timo this lease it in offect, mey remove said atorage otructures, facilities, scalea, fences or algms or eny

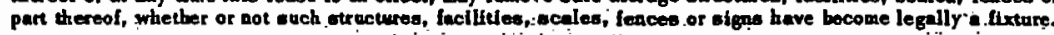

6. The Lessee ebali not wasigan this loses without the written consent of the Lessor. The Lessos, may, howorer, nublet. the siructures on the premises.leased heresuder, or any one-or moie of them for the term of the.lonse or any purt thereof upon

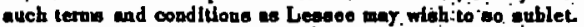

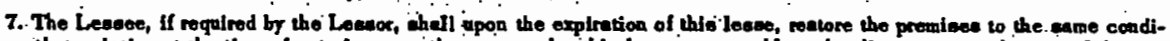

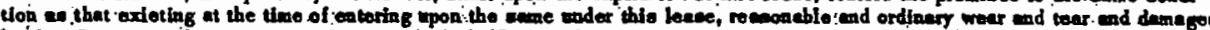
by - the olements or by circunetancess over which the Leesee bes no coatrol excepted:. Provided howiver, That If the Leseor a

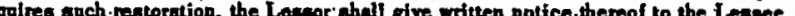

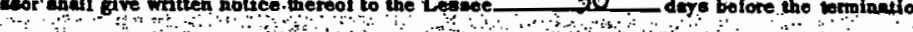

8. The Lessor grents and gives the Leseet the option a oonsideration of this laene-and for the further conalderation of

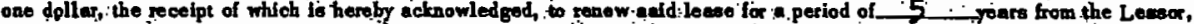

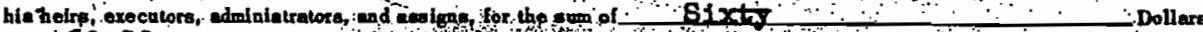
(s 60,00 ) per your.

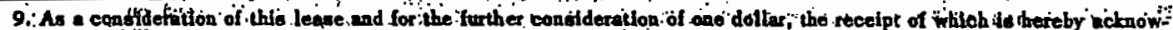

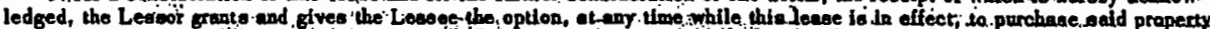

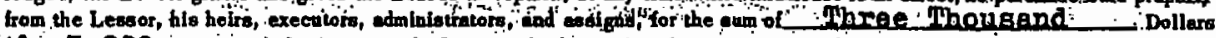

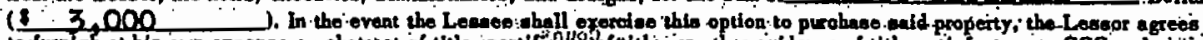

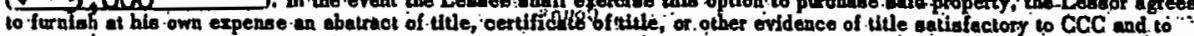

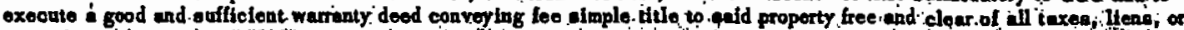

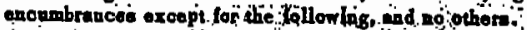


10. In the eveat any increased tax ansessment is made agahast the Lessor or the property by virtue of the erection of atora ge etructurei and facilitiea therean by the Lasaee, the Losaor agreen to coope rate folly to any conteat of auch increased asesement which the Leacee leels ghould be made. The Leasee agrees that the rental herounder jhall be adjusted upward by the amount of any euch increased tax aseecement which the Leseot and Laesoe matually a groe to be proper or which is determined to be logally valid in court proceedinge.

11. No member of or Delegute to Congreas or Realdent Commisaioner, abell be adgitted to any ehare or part of this leane or purchesé or to eny benefit that may arise therefrom, hut this provision ahall not be conatrued to oxtend to this leane or purchase if made with a corporation for lis gecerral beacilt.

12. The Legsor warrante that he has not employed any person to solicit ar sectre this lease apon any agreemeat for a commianion, percentege, brakerage, or contingent fee and that no avch coingideration or payment has been or will be made. Breach of this warranty ohell give CCC the right to enaul the leaee, or, in its discrotion, to deduct from the rentel or purchese price the emouat of asch carmission, perceatage, brokerage, or costingent fees. This warraty ahell not apply to coumlas tors pay -ble by the Lessor If the lease la secured or made through a bona-flde agent mainteised by the Lessor for the purpose of - lasaing or selling his property.

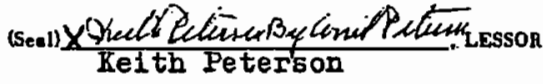
(Seal) LESSOR VITNESS
CONODTXX CREDTT CORPORATION, LESSEE

$$
\begin{aligned}
& \text { B. Olydel Oline } \\
& \text { Charmen, McPherson - ClJde I Cline } \\
& \text { Contracting Oflicer }
\end{aligned}
$$

\section{ACKNONLEDGKENI}

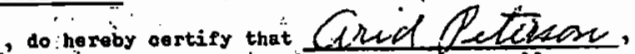
$I$, the exeouted the foregoing instrument, personaliy to where or they) exeouted the same as his (hor or

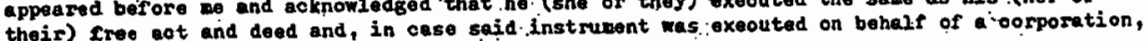
their) free oot and deed and, in case said instruwent mas oxeouted on behalf of oorporation,
that he. (she or they) as

As:

Wnit. (insert nade of offioer(s) and his (her or their) official

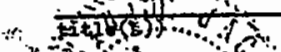

$=0 \%$, Gasi(were) duly authorized by the Board

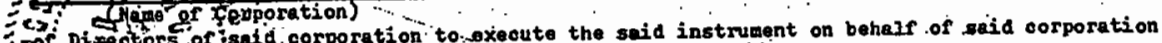
$=0$ Dise otion

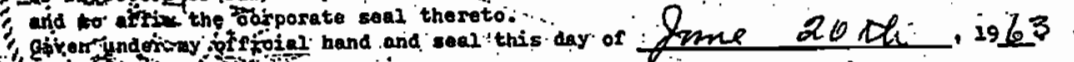

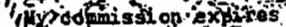

(1,

Het

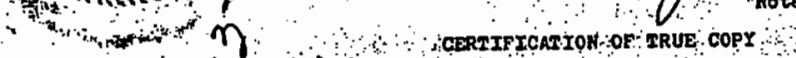
1963

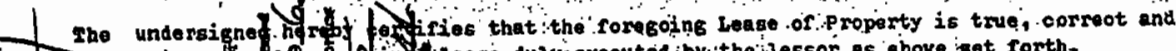

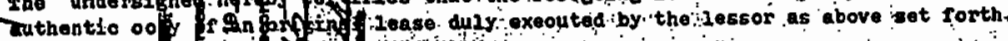

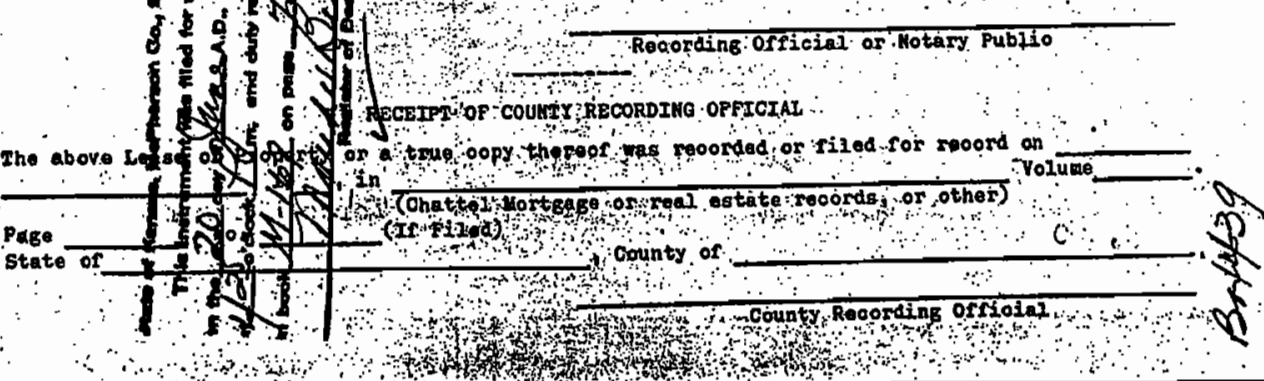




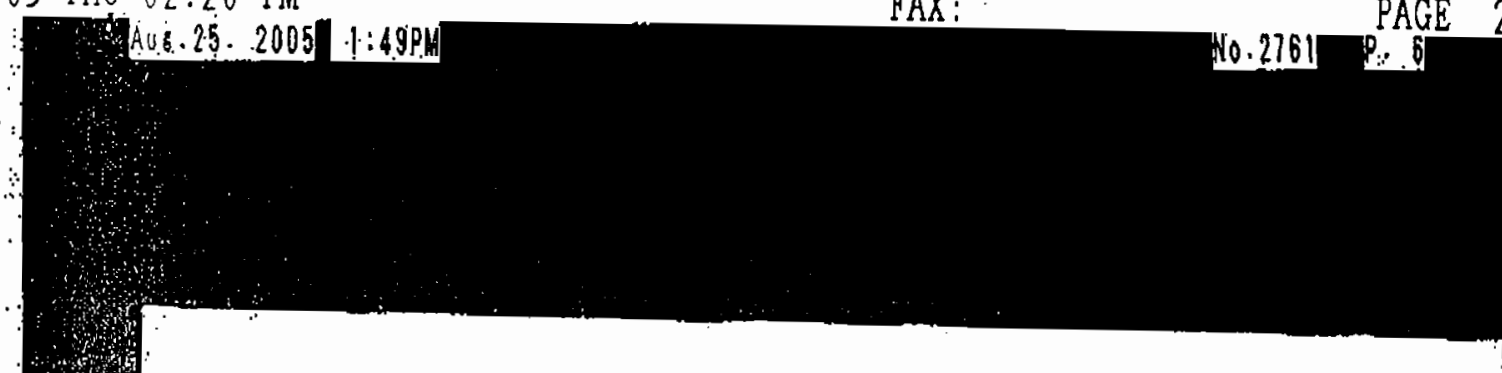

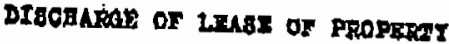

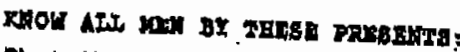

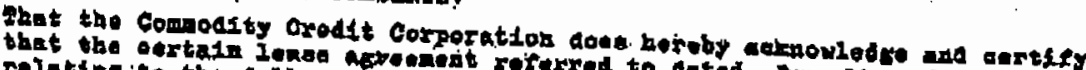

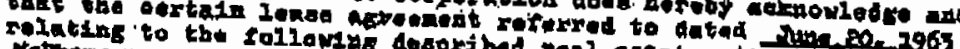

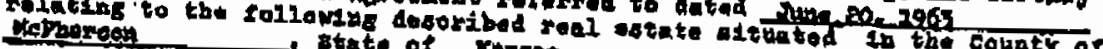
Bogdanter in the soutbents to wdti

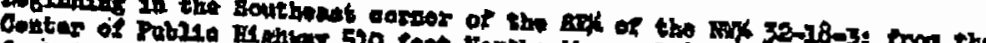

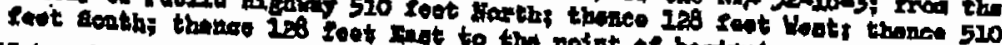

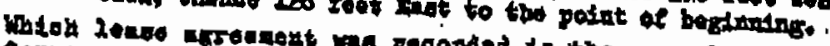

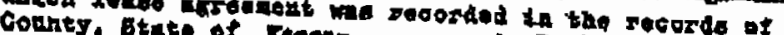

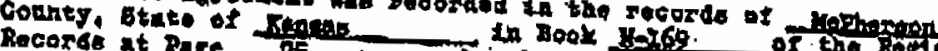

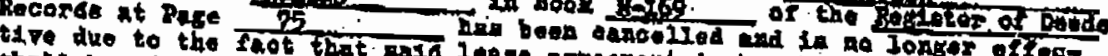

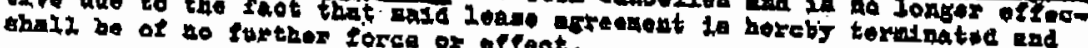

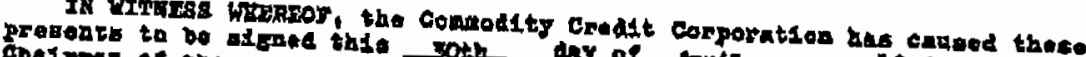

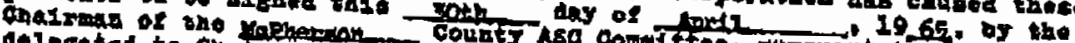

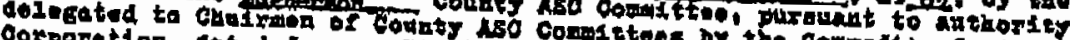

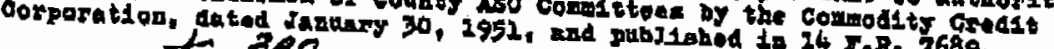

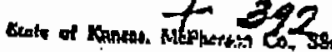

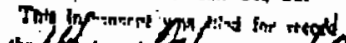

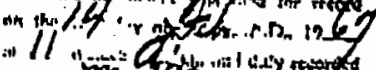

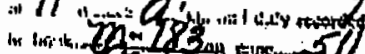

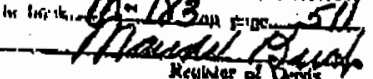

sfate or Retwer at berath

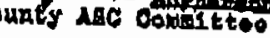

COWMODIT: CHFDIs CORFopumYor

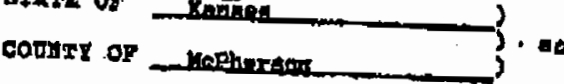

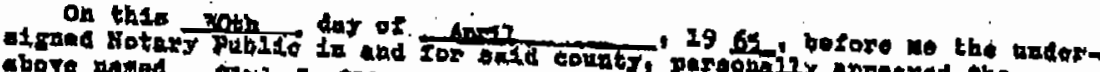

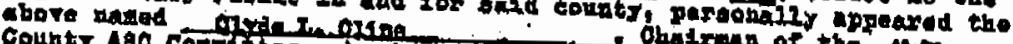

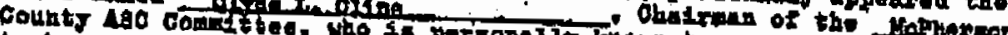

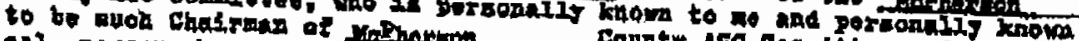

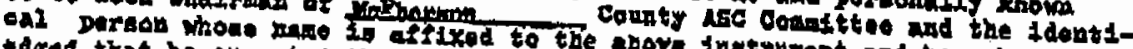

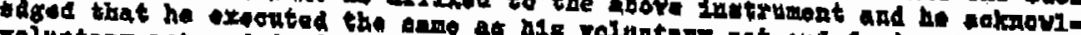

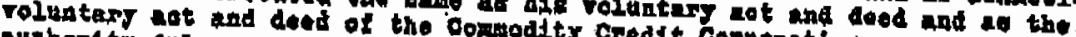
autbortty ally conterred upos him.

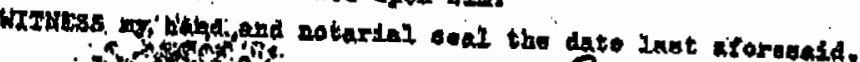

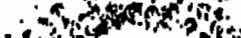

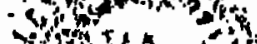

$+x_{2}+4 k+4 y$

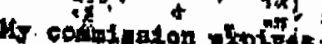

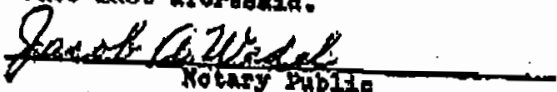

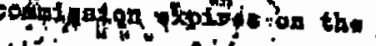
:5 $5 \%$ fip?

12 then dins of

yasth 1966. 
Pather

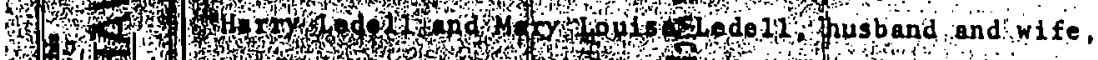

$4+3 \mathrm{~s}$

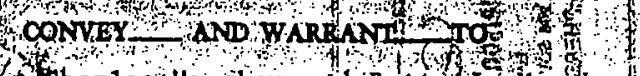

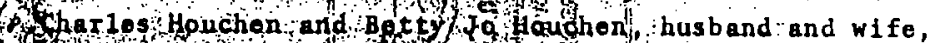

(1)

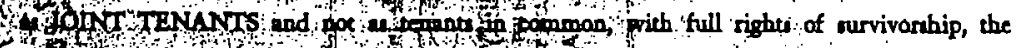

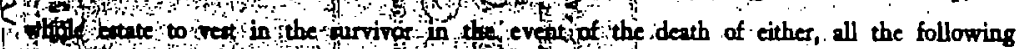
Achpod REALESTATE ini the County of MËPhers on

Sis

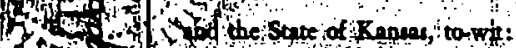

fengentions

然过

A tract of land in the Northeast Quarter (NE/4) of Section

Thirty-two (32), Township. Eighteen (18) South. Range Three

(3) West MePherson County Kansas, described as follows:

Beginning at the Northwest Corner of the NE/4 of said Section 32; ithence East along the Soction line 499.7 feet to the West: Ine of the right of-way of the Union Pacific Railroad thence Southwesterly along the. Hest line of said railroad right-of-way 438,0 feet; thence Northwesterly 441.6 feet to a point on the quarter section line dividing the NE/4 and the NW/4 of said Section 32,"which point is 387.0 feet South of the place of beginning; thence North to the place of beginning, containing.4.44 acres, nore or less.

for the sum of TEN and no/ 100 DOLLARS $(\$ 10.00)$ and other valuable considerations

EXCEPT AND SUBJECT TO:

\section{Dated February 5 \\ STATE. OF RANSAS, MCPHERSON COUNTY,}

BE IT REMEMBERED, That on this 5 th . day of Februaty, A. D. 1973 before me, the underrigaed, \& Notary Pub1ic; in and for the County and State aforeseid, ame

Harry Ledell and Mary Louise Lede11, hus band and wife;

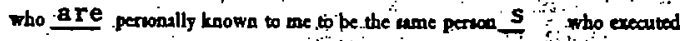

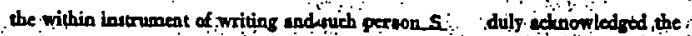
ecoction of the rame.

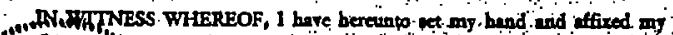

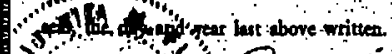

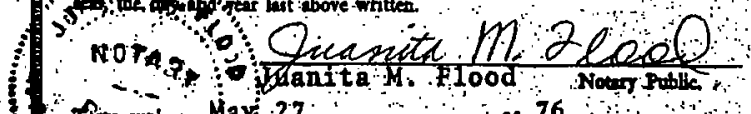

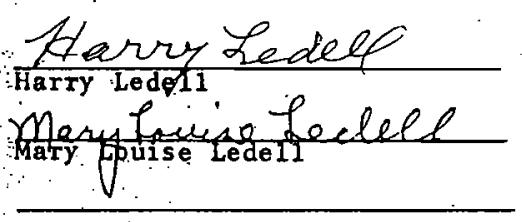

$\times 2335$

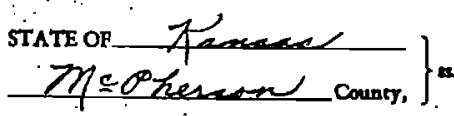

This instrument was filed for recoed on the

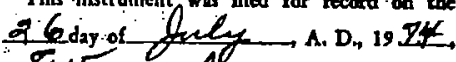
at $2 \mathrm{i} 15$.o'clock $A$. $\mathrm{M}$, and duly recorded in book 186 of asbee lak it page $4 / 1$

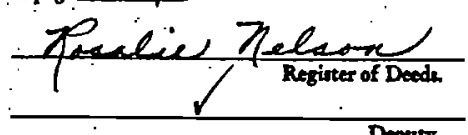

Feer 1 300 Deputy. 
JONNT TENANCY WARRANTY DEED (Following Kanen Sututory Wermety Form)

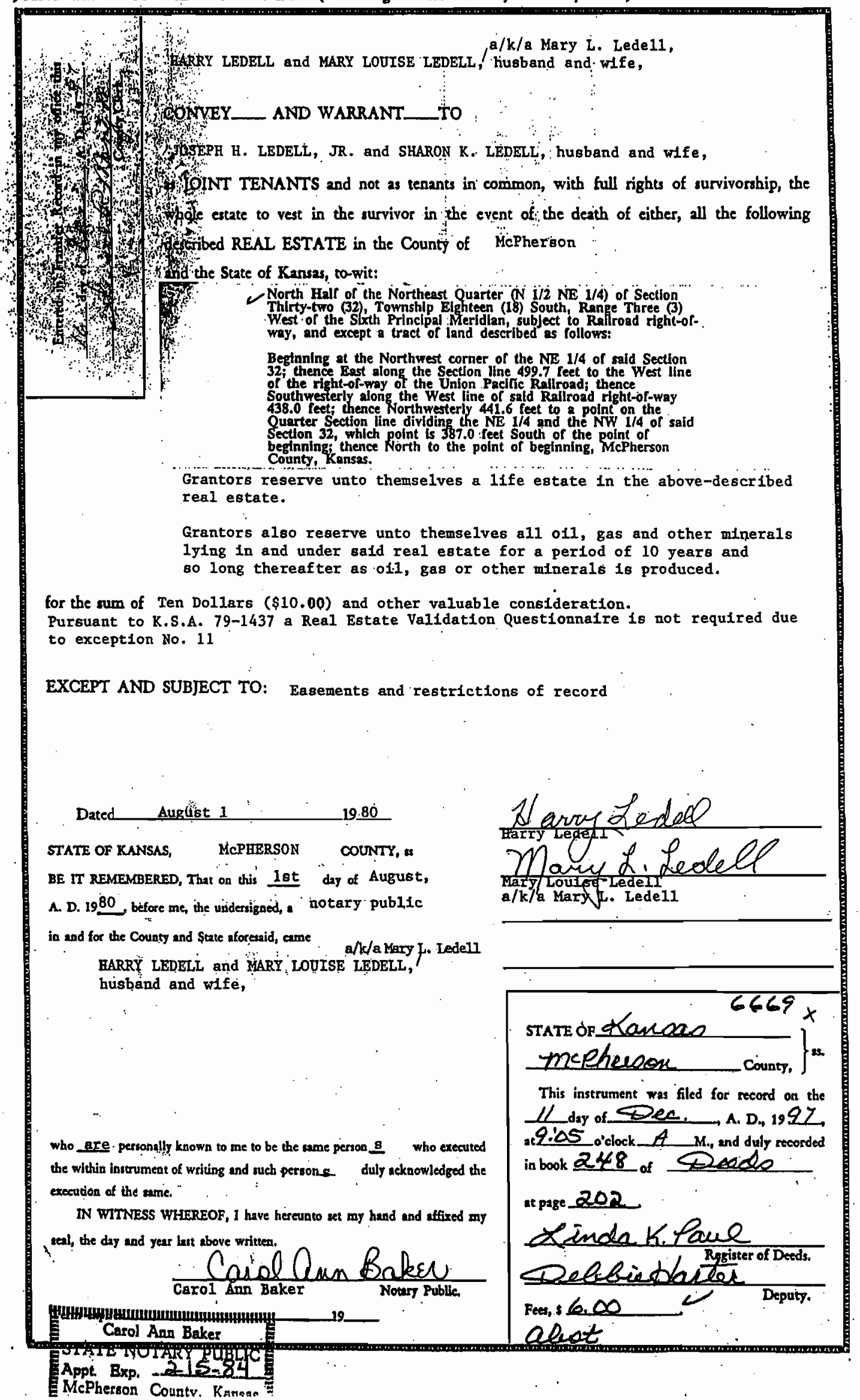




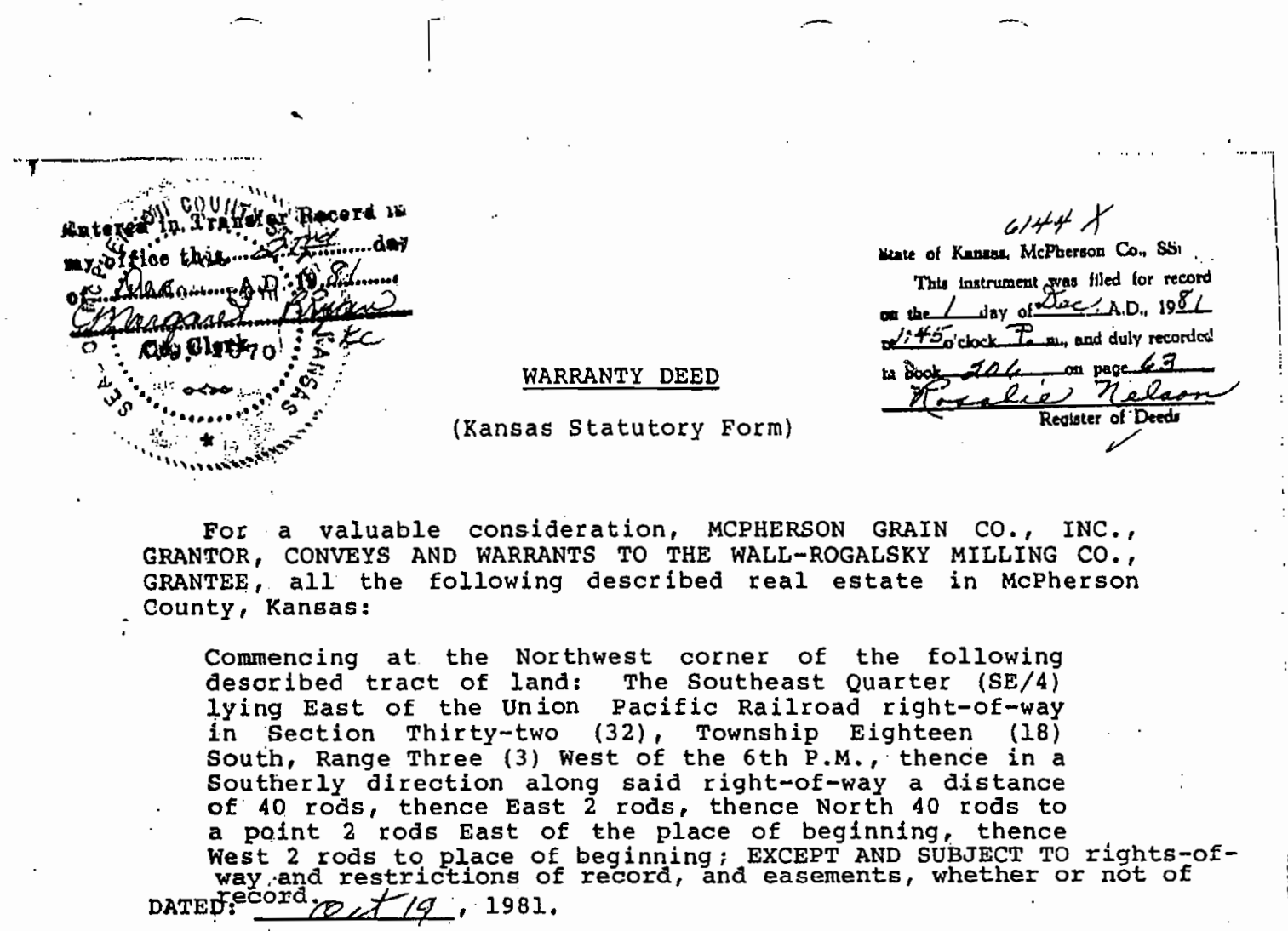

MCPHERSON GRAIN CO.; INC.

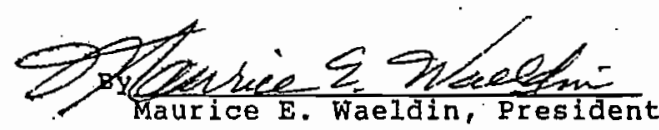

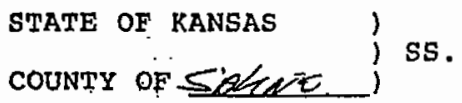

The foregoing instrument was acknowledged before me this 6 fth day of 2uber , 1981, by Maurice E. Waeldin, President of McPherson Grain Co., Inc., a Kansas corporation, on behalf of the corporation.

$-$
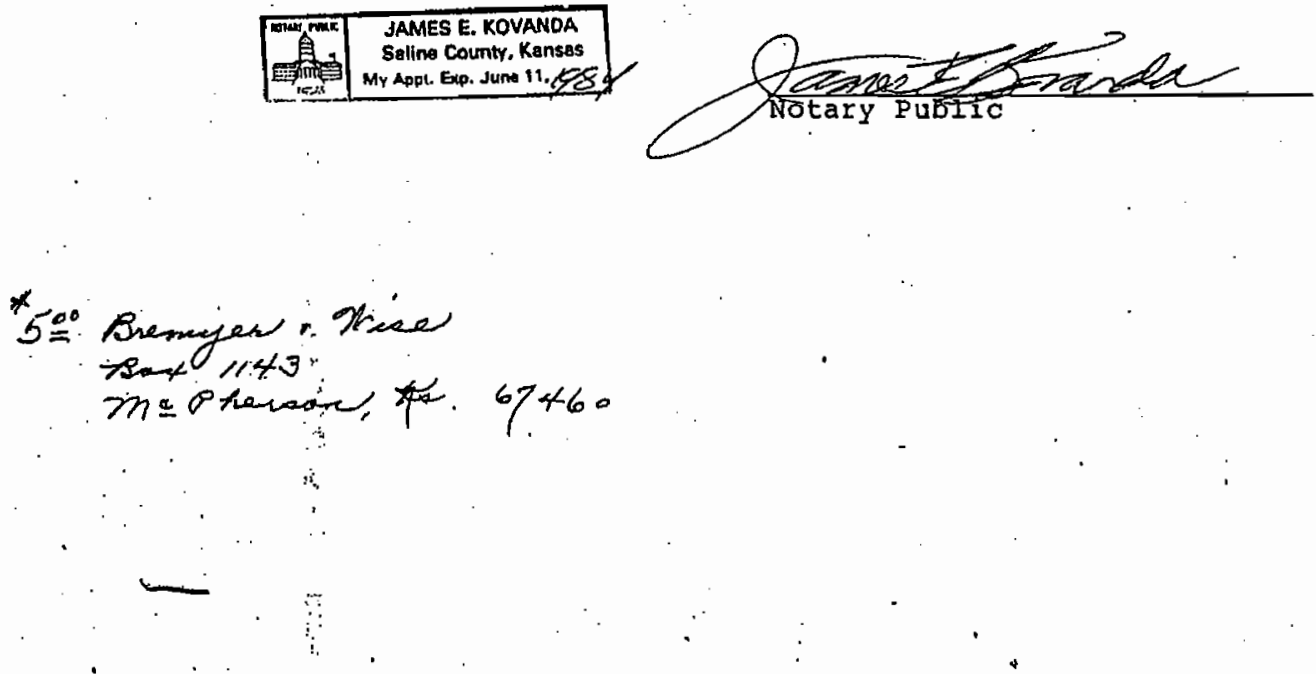


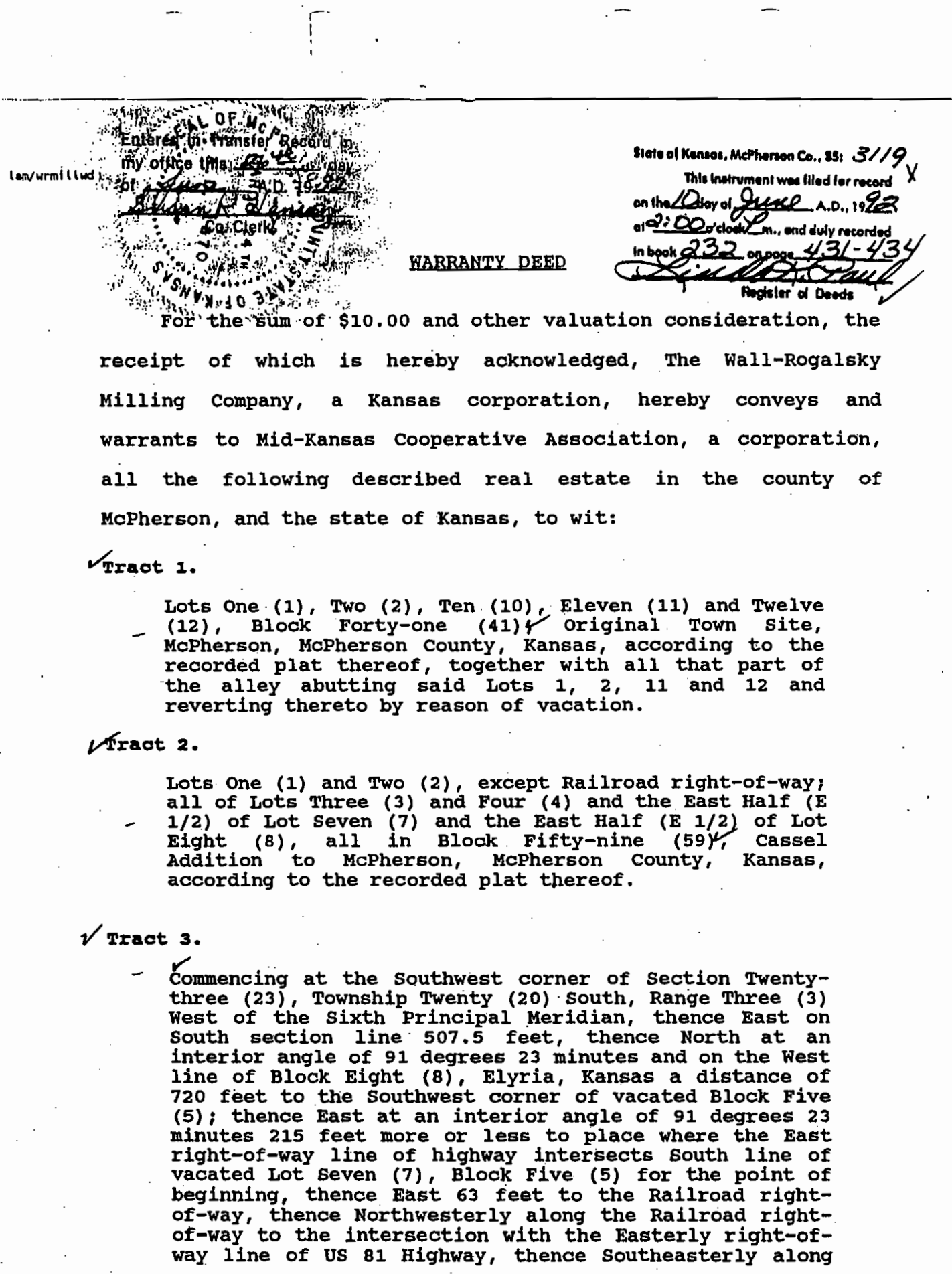


gaid Highway right-of-way to the point of beginning; gaid tract more particularly described as part of Lots Seven (7) and Eight (8), Block Five (5), and all of the alley abutting said lots as originally platted, lying between the East highway right-of-way line and the West Railroad right-of-way line;

AND

That portion of the North Half $\left(N_{1 / 2}\right)$ of Third street as originally platted in Elyria, Kansas; which lies between US Highway 81 and the Missouri Pacific Railroad Company right-of-way and that portion of the South Half

(s $1 / 2$ ) of Third street as originally platted in Elyria, Kansas which lies directly North of Lots Eleven (1i) in Block Eight (8) between Us Highway 81 and the Missouri Paclfic Railroad Company right-of-way;

AND

All of Lots Eleven (11) and Twelve (12)., Block Eight

- (8) in Elyria, Kansas, excepting those portions of said lots which form a part of Us Highway 81 ;

AND

$\checkmark \quad$ The North Half (N 1/2) of the alley in Block Eight ( 8 ) in Elyria, Kansas, lying between US Highway 81 and the East line of said. Block 8 and the Hest Half (W 1/24 of Kennebec street in Elyria, Kansas, which lies between the Missouri Pacific Railroad Company right-of-way and the center line of the alley located in Block 8 in Elyria, Kansas;

AND

All of Lot Nine (9), Block Fifteen (15) in Elyria,

- Kansas, except land deeded for Highway purposes and except Railroad right-of-way, in McPherson county, Kansas.

Traot 4.

A part of the southeast Quarter (SE 1/4) of section Thirty-two (32), Township Eighteen (18) South, Range Three (3) West of the sixth Principal Meridian, described as follows:

Commencing at the intersection of a line drawn paraliel with and 133 feet Easterly from the center line of the main track of the Union Pacific Railroad Company, 


\begin{abstract}
measured at right angles to said center line, with a line drawn parallel with and 16.5 feet South of the North line of said $S E 1 / 4$, section 32 , thence southwesterly along a line drawn parallel with and 133 feet Easterly from said main track center line when measured at right angles 40 rods; thence Easterly at a right angle from said main track center line 2 rods; thence Northeasterly along a line parallel with and 166 feet Easterly from said main track center line when measurad at right angles 40 rods, more or less, to a point 16.5 feet South of the North line of said SE 1/4, Section 32; thence Hest Parallel with the North line of said SE $1 / 4$, Section 32,2 rods, more or less, to the point of beginning, in McPherson County, Kansas.
\end{abstract}

Tract 5 .

Lots one (1), Two (2), Three (3), seventeen (17) and Eighteen (18), Block Fourteen (14), Windom, Mopherson

- County, Kansas, according to the recorded plat thereof;

AND

Lots one (1) and Two (2), Block Four (4), Windom, McPherson county; Kansas, according to the recorded plat thereof.

AND

Lots one (1), Two (2), Fifteen (15), Sixteen (16), Seventeen (17) and Elghteen (18), Block Fifteen (15), windom, MaPherson county, Kansas, according to the recorded plat thereof.

except and subject to:

(a) Easements, licenses, rights-of-way, restrictions and reservations of record, lncluding oil, gas and mineral reservations, if any, of record;

(b) The rights of the public or third parties in and to any roads, highways or rights-of-way across or adjacent to said real property; 


\section{4}

(c) Presently existing and enforceable licenses, rights-of-way and easements, if any, for public utilities, whether or not of record;

(d) Defects of title to lands of third parties over which easements, if any, are to be conveyed by Seller as appurtenances to said real estate;

(e) Liens of public authorities, if any, for property taxes or assessments not yet due and payable; and

(f) All applicable zoning or land use statutes, ordinances, rules and regulations.
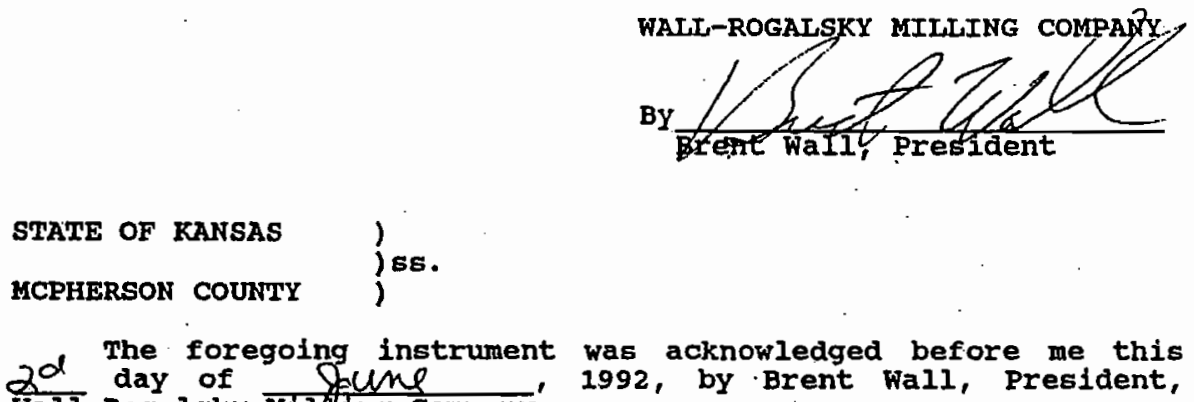

My appointment expires:

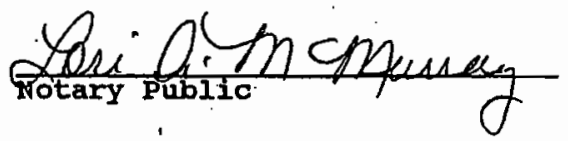

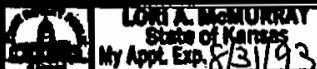

12.00

nid-Y) Cooperatios Wase 


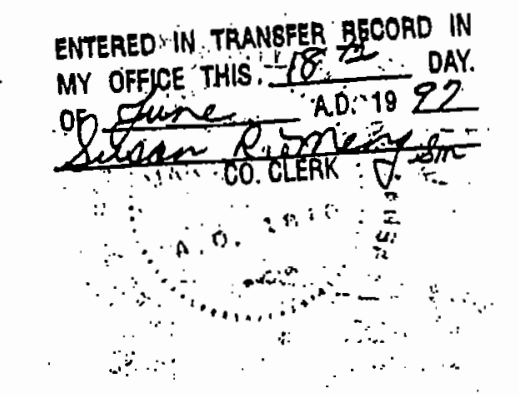

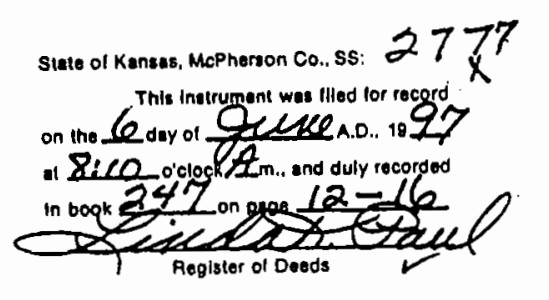

$399-60$

\section{CORRECTION DONATIVE QUITCLAIM DEED}

UNION PACIFIC RAILROAD COMPANY, a Utah corporation, Grantor, does hereby DONATE, REMISE, RELEASE and forever QUITCLAIM unto THE CENTRAL KANSAS CONSERVANCY, INC., a Kansas non-profit corporation, Grantee, and unto its successors and assigns forever, all of Grantor's right, title, interest, estate, claim and demand, both at law in equity, of, in, and to the real estate (the "Property") on that portion of Grantor's McPherson Branch from Grantor's Milepost 34.0 (formeriy MPRR Milepost 518.0) near McPherson, Kansas, to Grantor's Milepost 21.4 (formerly MPRR Milepost 530.6) near Lindsborg, Kansas, as such Property is located in McPherson County, Kansas, and as more particularly described in Exhibit A, hereto attached and hereby made a part hereof, reserving unto Grantor, its employees. agents, successors or assigns the right to enter on the Property for the purpose of appraising the value of the Property for a period of ninety $(90)$ days after conveyance of the Property to Grantee.

This deed is made SUBJECT TO :

1. All of the terms and conditions contained in that certain Line Donation Contract between Grantor and Grantee dated April 16, 1997.

2. The terms and conditions contained in the Decision and Notice of Interim Trail Use or Abandonment served by the interstate Commerce Commission on September 28, 1995, in Docket No. AE-33 (Sub-No. 89X) which authorized the Grantor to discontinue operation over the line of railroad described above, and also the rail-bank interim trail use condition imposed by the ICC in said Decision pursuant to Section 8(d) of the National Trails System Act, 16 U.S.C. § 1247(d).

3. All other conditions, restrictions, covenants, reservations, easements, rights and encumbrances affecting the Property, whether recorded or unrecorded. 
THIS QUITCLAIM DEED IS MADE ON AN "AS IS, WHERE IS". BASIS

WITHOUT ANY WARRANTIES OR REPRESENTATIONS OF ANY KIND OR NATURE WHATSOEVER, EXPRESS OR IMPLIED, CONCERNING THE CONDITIONS OF THE PROPERTY, AND GRANTEE HEREBY SPECIFICALLY WAIVES ANY IMPLIED WARRANTIES PROVIDED FOR BY KANSAS LAW (IF ANY), INCLUDING ANY AND ALL WARRANTIES REGARDING FITNESS FOR ANY PARTICULAR USE OR PURPOSE WHATSOEVER.

This Deed is given in correction of and substitution for a certain Donative Quitclaim Deed dated April 16, 1997 given by Grantor to Grantee, and filed for record on April 18; 1997 in Book 246, Pages 617-657 in the Deed Records of McPherson Counity, Kansas. Any inconsistency between the terms of that deed, and the terms of this deed shall be resolved in accordance with the terms of this deed with the exception of the map which is recorded on Pages 622-657. This deed shall be deemed to be controlling and to supersede that deed.

IN WITNESS WHEREOF, the Grantor has caused this Deed to be duly executed as of the $30^{\text {th }}$ day of May 1997.

$$
\text { Attest: }
$$

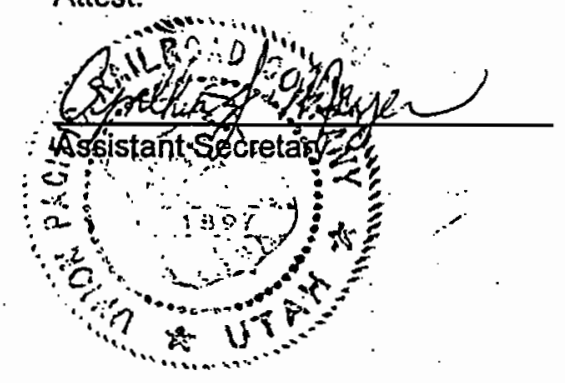

\section{UNION PACIFIC RAILROAD COMPANY}

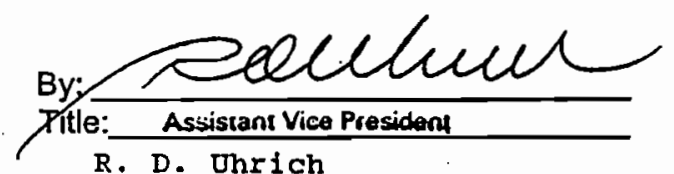

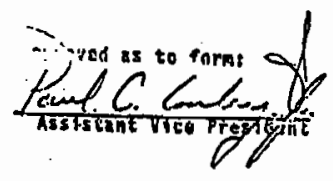




\section{ACKNOWLEDGMENI}

\section{STATE OF NEBRASKA ， COUNTY OF DOUGLAS )

$$
\text { iss. }
$$

On this 30 day of May 1997 before me, a Notary Public in and for said County and State, personally appeared ared R. Whisich and Cunthia Meyes. who are the and the Assistant Secretary, respectively, of Union Paclfic Railroad Company, a Utah corporation, and who are personally known to me (or proved to me on the basis of satisfactory evidence) to be the persons whose names are subscribed to in the within instrument, and acknowledged to me that they executed the same in their authorized capacities, and that by their signatures on the instrument the persons, or the entity upon behalf of which the persons acted, executed the instrument.

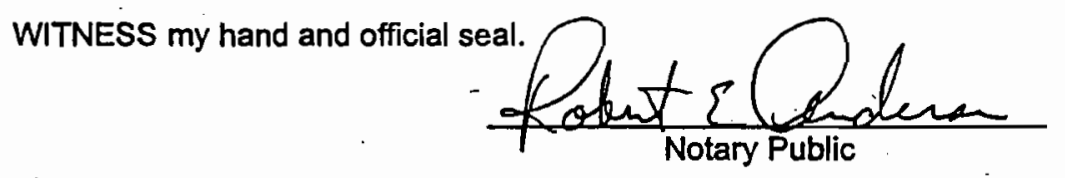

(Seal)

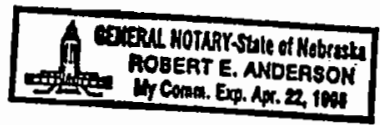




\author{
UNION PACIFIC RAILROAD COMPANY \\ UPRR MP 21.4 (MPRR MP 530.6) to UPRR MP 34.0 (MPRR MP 518.01 \\ McPherson Branch \\ McPherson County. Kansas

\section{EXHIBIT "A"}

All right, title, and interest in and to that portion of the right of way of the McPherson Branch of the Union Pacific Railroad Company as formerly constructed and operated, in, over, and across the following legal subdivisions:

\begin{tabular}{|c|c|c|c|c|}
\hline SUBDIVISION & SECTION & IOWNSHIP & RANGE & MERIDIAN \\
\hline$E Y_{2}$ & 20 & 175. & $3 W$. & 6TH - \\
\hline$E^{1 / 2}, S E 1 / 4$ SW $1 / 4$ & 29 & 175. & $3 W$. & 6TH. \\
\hline$W / 2$ & 32 & 175. & $3 W$, & 6TH- \\
\hline E $1 / 2$ SE $1 / 4$ & 31 & 175. & $3 W$. & 6TH - \\
\hline W1/2W1/2 & 5 & $18 S$. & $3 W$. & 6TH - \\
\hline NE $1 / 4$ NE $1 / 4$ & 6 & 185. & $3 W$. & 6TH, \\
\hline W $1 / 2$, SW $1 / 4$ SE $1 / 4$ & 8 & $18 S$. & $3 W$. & 6TH. \\
\hline W $1 / 2 E 1 / 2$ & 17 & $18 S$. & $3 W$. & 6TH. \\
\hline W $1 / 2 E 1 / 2$ & 20 & $18 S$. & $3 W$. & 6TH \\
\hline W $1 / 2 E^{1 / 2}$ & 29 & $18 S$. & $3 W$. & 6TH - \\
\hline$W^{1 / 2} E^{1 / 2}$ & 32 & 185. & $3 W$. & 6TH - \\
\hline ALL & 5 & 195. & $3 W$. & 6TH - \\
\hline$E^{1 / 2} W 1 / 2$ & 8 & 195. & $3 W$. & 6TH. \\
\hline ALL & 17 & $19 S$. & $3 W$. & 6TH- \\
\hline
\end{tabular}

Also that portion of the SEY of Section 17, Township 17 South, Range 3 West ofthe Sixth Principal Meridian in McPherson County, Kansas, lying between the south line of said Section 17 and a line drawn at right angles through the centerline of the main track of said McPherson Branch, said line being 262.7 feet northerly, measured along said centerline of the main track, from said south line of Section 20.

Also that portion of the N/\% of Section 20, Township 19 South, Range 3 West of ${ }^{-}$ the Sixth Principal Meridian in McPherson County, Kansas, lying between the north line of said Section 20 and $a$ line drawn at right angles through the centerline of the main track of said McPherson Branch, said line being 1,247.3 feet southerly, measured along said centerline of the main track, from said north line of Section 20.

Excepting therefrom all of that certain 100 foot wide strip of land situate in NW $1 / 4$ SE $1 / 4$ of Section 32, Township 18 South, Range 3 West of the Sixth Principal Meridian in McPherson County, Kansas, said strip being all of that certain strip of land conveyed by Warranty Deed dated December 19, 1889, from Oscar Sellberg to Salina \& Northwestern Railway Company (predecessor to Union Pacific Railroad Company) recorded on February 3,1890, in Book 40 at Page 49, McPherson County, Kansas.

Also excepting therefrom all of that certain 100 foot wide strip of land situate in the * NW $\%$ SE $1 / 4$ of Section 32, Township 18 Socith, Range 3 West of the Sixth Principal Meridian in McPherson County, Kansas, said strip being all of that certain strip of land conveyed by Warranty Deed dated August 30,-1899, from Oscar Sellberg, et ux, to Union 
Pacific Railrosd Company recorded on March 28, 1900, in Book 48 at Page 390. McPherson County, Kansas.

Also excepting therefrom all of that certain 100 foot wide strip of land situate in the NW $1 / 4$ SE $1 / 4$ of Section 32, Township 18 South, Range 3 West of the Sixth Principal Meridian in McPherson County, Kansas, said strip being all of that certain strip of land conveyed by Warranty Deed dated June 3, 1904, from Oscar Sellberg, et ux, to Union Paclfic Railroad Company recorded on June 25, 1904, in Book 64 at Page 30, McPherson County, Kansas.

Office of Contracts \& Real Estate Omaha, Nebraska

March B, 1996

Revised: April 29, 1997

Written bY: LEF

MCPHERSN. LGL.

$399-60$

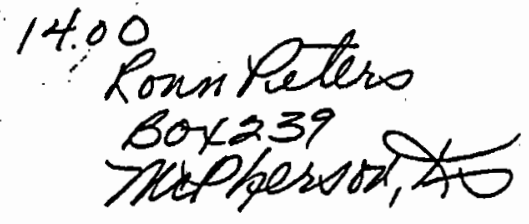




\section{6}

JOINT TENANCY WARRANTIY DEHED

Richard L. Howse, A single Person

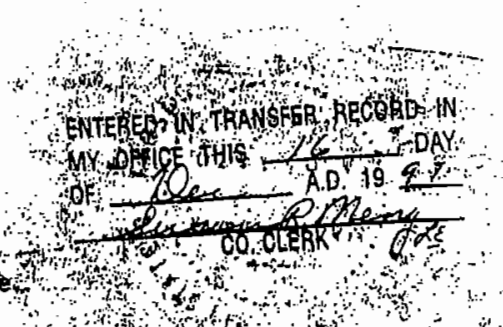

CONVEY(S) and WARRANT(5) TO

Joseph H. Ledell, Jr, and Sharon K. Ledell, husband and wifé

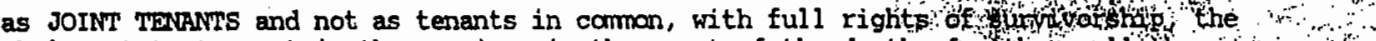
whole estate to vest in the survivor in the event of the death of efther, al the following described RERL ESTATE in the County of McPherson and the state of Kifisis'; ". to-wit:

The South Half of the Northeast Quarter (S $1 / 2$ NE 1/4) of Section Th1rty-two (32), Township E1ghteen (18) South, Range Three (3) West of the Sixth Principal Meridian, Subject to Ra1lroad right-of-way, and except a tract of Land described as follows: Beginning on the East line of the right-of-way of Salina and South Western Raflway Company $161 / 2$ feet North of the South 11ne of the NE 1/4 of sald Section 32, Thence East $100 \mathrm{feet}$, thence in a Northerly difection parallel to right-of-way $3791 / 2$ feet, thence West 100 feet to right-of-way of bald Sallna and South Western Railway Company, thence South along sald right-of-way $3791 / 2$ feet to the point of beginning, McPherson County, Kansas.

for the sum of TEN DOLEARS AND 00 CENTS and other good and valuable consideration EXCEPT AND SUBJECT TO:

, Easements and Restrictions of Record, if any.

DATED: December 9, 1997

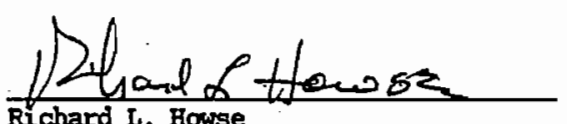

Richard L. Howse

STATE OF KANSAS, RILEF CONNT, BS:

BE IT RAMEMBERED, that in this 9 th day of December A.D. 1997, before me, the undersigned, a notary public in' and for the county and state aforesaid, came

Richard L. Howse, A single Person

who is personally known to me to be the same person who executed the within instrument of writing and such person duly. acknowledged the execution of the sarine.

IN WITTESS WHIRRBOF, I have hereunto set my hand and affixed ny seal, the day angyyear last above written.

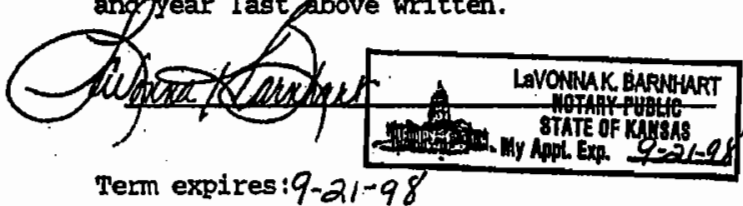

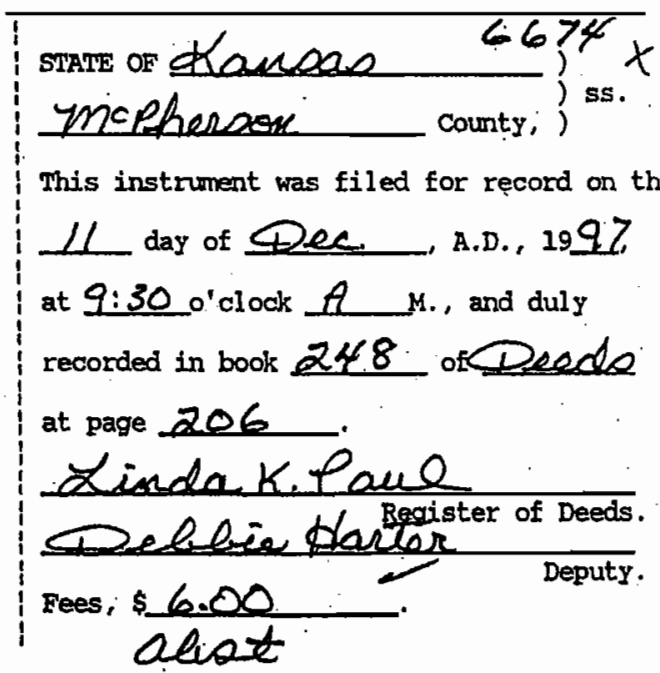




\section{. . . 1 \\ JOINT TENANCY WARRANTIY (KANSAS STATUTORY WARRANTY FORM)}

wer

Russell R. Howse and Nancy Howse, Husband and Wife CONVEY(5) and WARRANT(5) TO

Joseph H. Ledell, Jr. and Sharon K. Ledell, husband and wif\&;

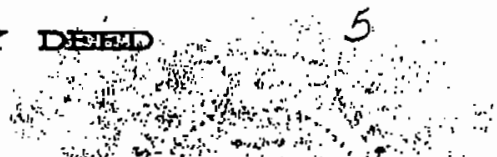

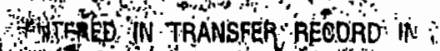

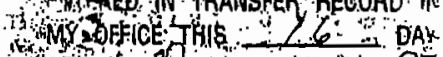
ToE (1)

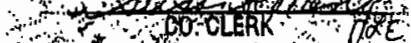

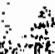
as JOINT TENANTS and not as tenants in common, with full rights of "survivorship", the whole estate to vest in the survivor in the event of the death of either, all the following described REAL ESTATE in the County of MCPherson and the state of Kansas, to-wit:

The Township Eighteen (18) South, Range Three. (3) West of the S1xth Princlpal Meridian, subject to Rajlroad right-of-way, and except a tract of land described as follows:

Beginning on the East line of the right-of-way of Salina and South Western Ra1lway Company 16 1/2 feet North of the South line of the NE $1 / 4$ of said Section 32 , thence East 100 feet, thence in a Northerly direction parallel to right-of-way $3791 / 2$

feet, thence West 100 feet to right-of-way of sald Salina and South Western RaIlway Company, thence South along sald rlght-of-way $3791 / 2$ feet to the point of beginning, MePherson County, Kansas:

for the sum of TWN DOLCARS AND 00 CENTS and other good and valuable consideration EXCEPT AND SUBJECT TO:

Easenents and Restrictions of Record, if any.

DATED: December $\stackrel{\&}{\ominus}, 1997$

STATE OF KANSAS, RILEY OOUNTY,SS:

BE IT REMBMBERED, that on this $8^{\text {th }}$ day of Decerber A.D. 1997, before me, the undersigned, a notary public in and for the county and state aforesaid, caine

Russell R. Howse and. Nancy $T$. Howse, Husband and wife who are personally known to me to be the same persons who executed the within instrument of writing and such persons duly acknowl edged the exiecution of the same.

IN WITHESS HHEREOF, I have hereunto set my hand and affixed my seal, the day and year last above written.

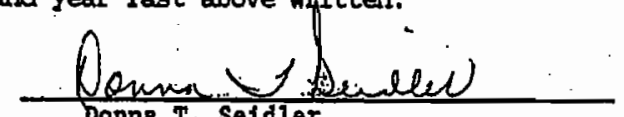

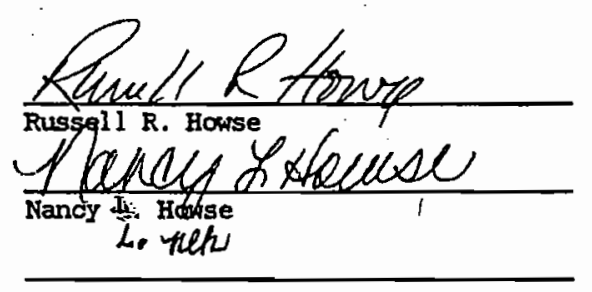

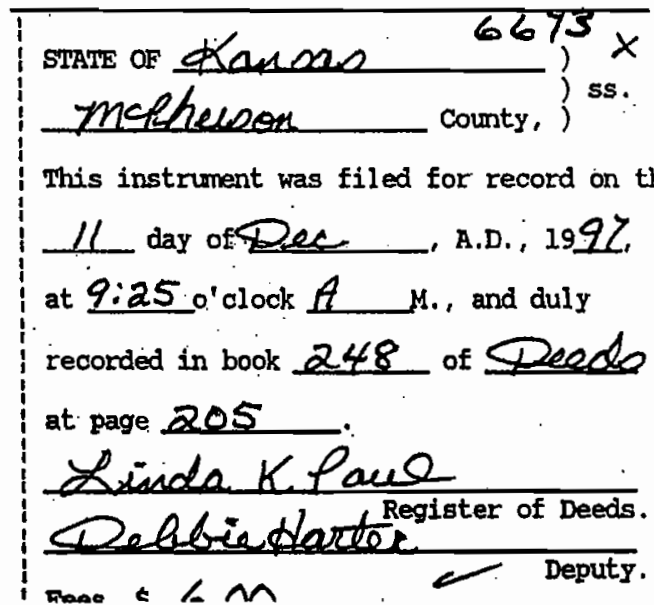




\section{4}

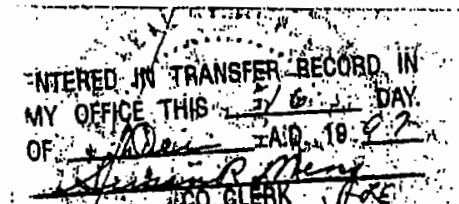

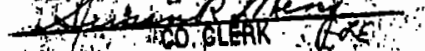

a

THS INDENTyke made this $8^{7 / h}$ day of December 1997 by and between The Trust Company of

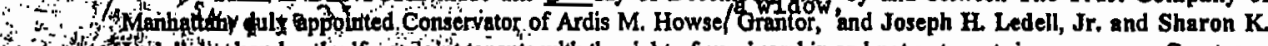

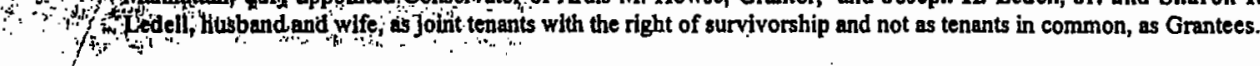

Grantor, by virtue of an Order authorizing the sale of real estate issued by the District Court of Riley County, Kansas, on the 25th day of June, 1997, having sold the real estate hereinafter described, in compliance with said order, and being duly confirmed as provided by law, in consideration of the sum of Seventy Three Thousand One Hundred and Fifty Dollars and zero cents $(\$ 73,150.00)$, which is not less than three-fourths of the appraised value of the real estate hereinafter described, does by these presents grant, bargain, sell, and convey to Joseph $H$. Ledell, Jr. and Sharon K. Ledell as joint tenants with the right of suryiygrship and not as tenants in common, their heirs and assigns, the right, title and interest of Ardis M. Howse/dischiarged from all liability for her debts, in and to the following-described real estate located in McPherson County, Kansas:

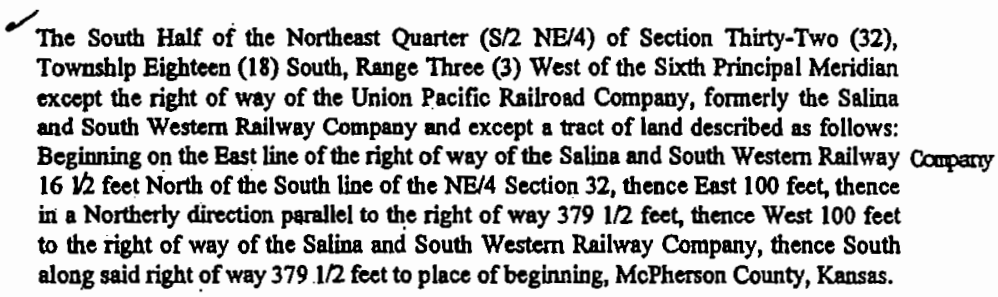

TO HAVE AND HOLD the above-described real estate, together with all and singular the tenements, hereditaments and appurtenances thereunto belonging or in anywise appertaining, forever.

IN WITNESS WEEREOF, Grantor has hereunto set its hand this 874 ,day of December, 1997.

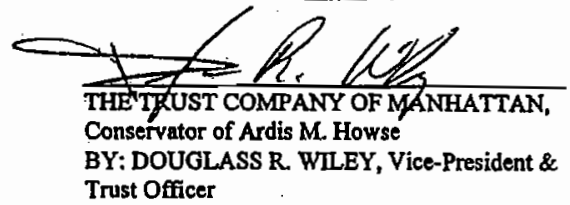

\section{STATE OF KANSAS, COUNTY OF RILEY, SS}

BE IT REMEMBERED that on this 8 day of December, 1997 before me, the undersigned, notary public in and for the county and state aforesaid, came Douglass $R$. Wijey Yice-President and Trust Officer of The Trust Company of Manhattan, Conservator of Ardis M. Howse/who Is personally known to me to be the same person who executed the above Conservator's Deed and such person duly acknowledged the execution of the same.

IN TESTIMONY WHEREOF, I have hereunto set my hand and affixed my official seal the day and year last above written.

$$
\text { My appointment expires: } 14,1998
$$

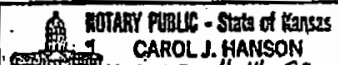

\subsection{0

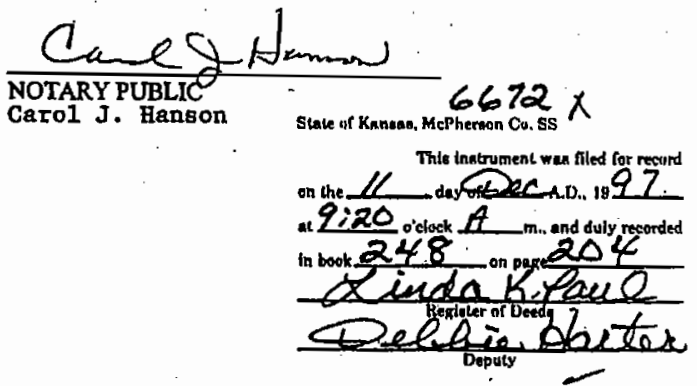


and

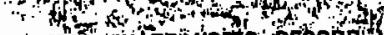

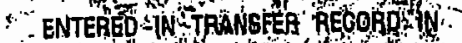

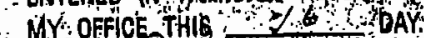

$\therefore$ of $20 \% 18$

or $d x$

$\frac{1}{20}$

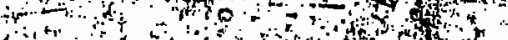

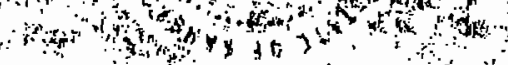

QUIT CLAIM DEED

Härry Ledell, a single person,

QUIT CIAIMS TO

Joseph H. Ledell, Jr, and Sharon $K$. Ledell, husband and wife, as JOINT TENANTS and not as tenants in common, with full rights of survivorship, the whole estate to vest in the survivor in the event of the death of either,

all of his right, title and interest in and to the following described real estate located in McPherson County, Kansas:

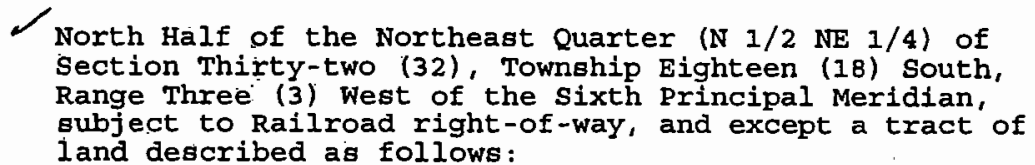

Beginning at the Northwest corner of the NE $1 / 4$ of said Section 32: thence East along the section line 499.7 feet to the West line of the right-of-way of the Union Pacific Railroad; thence Southwesterly along the West line of said Rail road right-of-way 438.0 feet; thence Northwesterly 441.6 feet to a point on the Quarter Section i-ine dividing the NE $1 / 4$ and the $N W 1 / 4$ of said Section 32, which point is 387.0 feet south of the point of beginning; thence North to the point of beginning, MCPherson County, Kansas;

"Pursuant to K.S.A. 79-1437, a Real Estate Sales Validation Questionnaire is not required due to Exception No. 12."

EXCEPT AND.SUBJECT TO: Easements, restrictions and reservations of record, if any.

FOR AND IN CONSIDERATION of the sum of One Dollar $(\$ 1.00)$ and termination of life estate and mineral reservation.

DATED this 9th day of December, 1997.

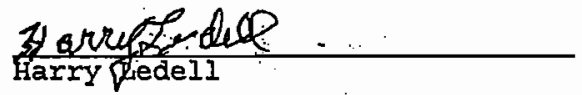

STATE OF KANSAS, COUNTY OF MCPHERSON, SS:

BE IT REMEMBERED, that on this 9th day of December, 1997 before me, the undersigned, a Notary Public, duly commiseioned in and for the county and state aforesaid, came Harry.Ledell, a single person, who i.g personally known to me to be the same perion:who executed the within instrument of writing and such person duly ackinowledged the execution of the same.

IN.WITNESS WHEREOF, I have hereunto set my hand and official seal on the day and year last above mritten.

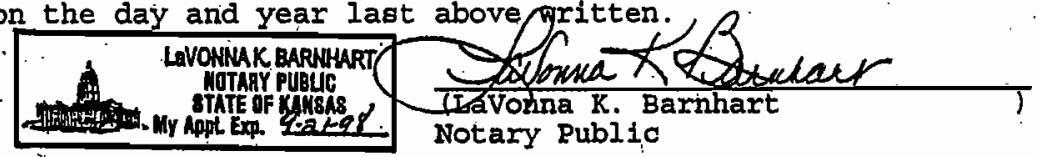

My commission expires: September 21, 1998 


\section{RIGHT-OF-WAY-EASEMENT}

KNOW ALL MEN BY THESE PRESENTS, that Charles Houchen and Betty Jo Houchen, husband and wife, hereinafter called Grantors, in consideration of one dollar (\$1.00) and other good and valuable consideration paid by Rural Water District No. 6 , hereinafter called the Grantee, the receipt and sufficiency of which is hereby acknowledged, does hereby grant, bargain, sell, transfer, and convey to said Grantee, its successors, and assigns, a perpetual easement with the right to erect, construct, install and lay and thereafter use, operate, inspect, repair, maintain, replace and remove over and across the following land owned by Grantors in McPherson County, Kansas:

\section{See attached Exhibit A.}

Together with the right of ingress and egress over Grantors' adjacent lands for the purposes for which the above-mentioned rights are granted. The easement hereby granted shall not exceed thirty feet $\left(30^{2}\right)$ in width, the centerline thereof to be located across said land as finally laid and installed.

The consideration recited herein shall constitute payment in full for all damages sustained by Grantors by reason of the installation of the structures referred to herein and the Grantee will maintain such easement in a state of good repair and efficiency so that no unreasonable damages will result from its use of Grantors ${ }^{2}$ premises. This Agreement together with other provisions of this grant shall constitute a covenant running with the land for the benefit of the Grantee, its successors, and assigns. The Grantors covenant that they are the owners of the above-described lands and that said lands are free and cleat of all encumbrances and liens except the following:

IN WITNESS WHEREOF, the said Grantors have executed this instrument this $25^{\text {th }}$ day of Dune, 2002

$$
\text { fenctis }
$$

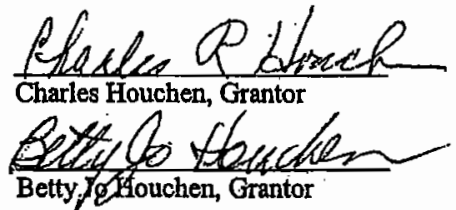

STATE OF KANSAS, COUNTY OF MCPHERSON: ss.

This instrument was acknowledged before me on Auns 25,2002 , by Charles Houchen and Betty Jo Houchen, husband and wife, Grantors.

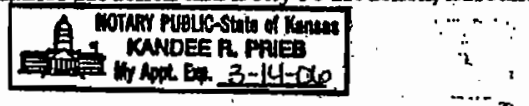

My appointment expires:

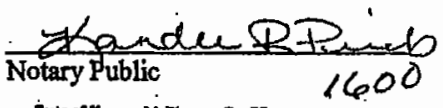

Buto of Keman, Mapherton Co.88

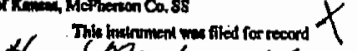

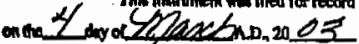

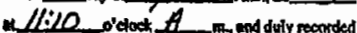
in brook $21-3 / 18$ mm $213-214$ Dindatiche Deledieghentex

\section{1}

Dipuly 


\section{Exhibit A.}

A tract of land in the Northeast Quarter (NE/4), Section Thirty-two (32), Township Eighteen (18), Range Thiree (3), West of the Sixth Principal Meridian, described as follows:

Beginning at the Northwest corner of the NE $1 / 4$ of said Section 32; thence East along the Section Iine 499.7 feet to the, West line of the right-of-way of the Union Pacific Railroad thence southwesterly along the West line of said Railroad right-of-way 438.0 feet; thence Northwesterly 441.6 feet to a point on the Quarter section line dividing the $N E 1 / 4$ and the $N W 1 / 4$ of said Section 32, which point is 387.0 feet south of the point of beginning; thence . North to the point of beginning, McFherson County, Kansas; 
DUPLICATE ORIGINAL

[McPherson County, Kansas]

PERPETUAL EASEMENT DEED

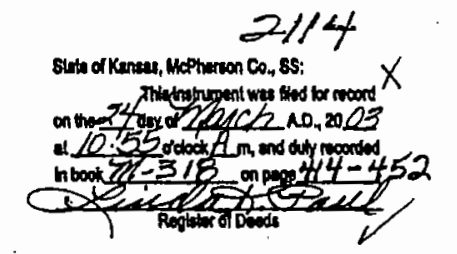

THIS EASEMENT DEED, made as of the $30^{\text {th }}$ day of September, 2002 , by UNION PACIFIC RAILROAD COMPANY, a Delaware corporation, having an office at 1416 Dodge Street, Omaha, Nebraska 68719, Grantor, AT\&T CORP., Grantee, a New York corporation, having an office at 1200 Peachtree Street, N.E., Promenade One - Room 2015, Atlanta, GA 30309.

WINESSETH, that Grantor and Grantee have entered into an Easement Agreement dated as of February 25, 1988 (hereinafter the "Agreement") whereby Grantor has granted to Grantee rights to construct, operate, maintain and replace a

telecommunications transmission system on the property of the Grantor under the terms, provisions, and conditions contained in the Agreement, one of which is that Grantor shall grant to Grantee a perpetual easement in the form of this deed, the covenants of which touch and concern the land encumbered by this deed.

NOW, THEREFORE, for and in consideration of Ten Dollars $(\$ 10.00)$, the receipt of which is hereby acknowledged, and for other good and valuable consideration, Grantor, by these presents does hereby grant to Grantee a PERPETUAL EASEMENT as set forth in the Agreement in the locations situated in the County of McPherson, State of Kansas, as more particularly described in Pages 7-15 of Exhibit "A", attached hereto and by this reference made a part hereof.

DN WITNESS WHEREOF, the Grantor has caused these presents to be signed by its Vice President - Law and attested by its Assistant Secretary and its corporate seal to be hereunto affixed as of the day and year first herein written.

UNION PACIFIC RAILRÓAD COMPANY,

Attest:
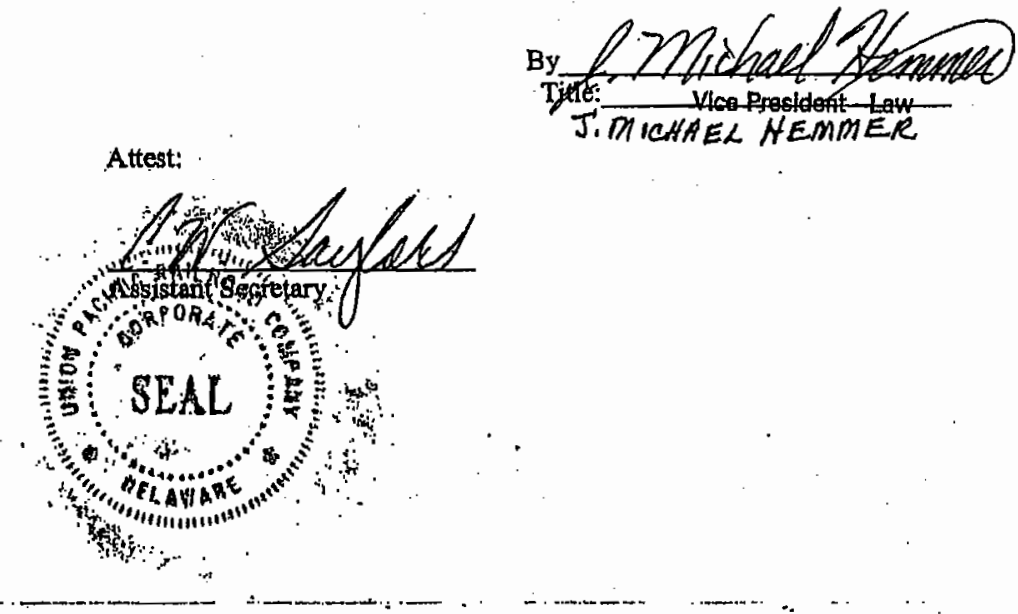


\section{Exhibit "B"}

Description of a Perpetual Easement granted to AT\&T Corp from Union Pacific Railroad dated September 30, 2002 and more particularly described in Exhibit " $A$ ".

The said Easement being located in and a part of the following Sections in McPhearson County, Kansas:

NE 1/4 Sec 3, T17S, R3W

$\sim \mathrm{NW} 1 / 4 \mathrm{Sec} 3$, T17S, R3W

-SW 1/4 Sec 3, T17S, R3W

$\sim \mathrm{SE} 1 / 4 \mathrm{Sec} 4, \mathrm{~T} 17 \mathrm{~S}, \mathrm{R} 3 \mathrm{~W}$

$\sim \mathrm{NE} 1 / 4 \operatorname{Sec} 9, \mathrm{~T} 17 \mathrm{~S}, \mathrm{R} 3 \mathrm{~W}$

$\sim \mathrm{NW} 1 / 4 \mathrm{Sec} 9$, T17S, R3W

$\sim$ SW 1/4 Sec 9, T17S, R3W

- SE $1 / 4 \operatorname{Sec} 8, T 17 S, R 3 W$

$\sim \mathrm{NE} 1 / 4 \mathrm{Sec} 17, \mathrm{~T} 17 \mathrm{~S}, \mathrm{R} 3 \mathrm{~W}$

$\checkmark \mathrm{SE} 1 / 4 \mathrm{Sec} 17$, T17S, R3W

$\sim \mathrm{NE} 1 / 4 \mathrm{Sec} 20$, T17S, R3W

$\sim \mathrm{SE} 1 / 4 \mathrm{Sec} 20$, T17S, R3W

$\sim \mathrm{NE} 1 / 4 \mathrm{Sec} 29$, T17S, R3W

- SE 1/4 Sec 29, T17S, R3W

$\checkmark$ SW 1/4 Sec 29, T17S, R3W

$\sim \mathrm{NW} 1 / 4 \mathrm{Sec} 32, \mathrm{~T} 17 \mathrm{~S}, \mathrm{R} 3 \mathrm{~W}$

$\sim \mathrm{SW}$ 1/4 Sec 32, T17S, R3W

$\sim$ NW $1 / 4$ Sec 5, T18S, R3W

$\sim \mathrm{SW} 1 / 4 \mathrm{Sec} 5, \mathrm{~T} 18 \mathrm{~S}, \mathrm{R} 3 \mathrm{~W}$

NW 1/4 Sec 8, T18S, R3W

$\checkmark$ SW 1/4 Sec 8, T18S, R3W

$\sim$ SE 1/4 Sec 8, T18S, R3W

$\sim \mathrm{NE} 1 / 4 \mathrm{Sec} 17, \mathrm{~T} 18 \mathrm{~S}, \mathrm{R} 3 \mathrm{~W}$

$\checkmark \mathrm{SE} 1 / 4 \mathrm{Sec} 17$, T18S, R3W

$\sim \mathrm{NE} 1 / 4 \mathrm{Sec} 20, \mathrm{~T} 18 \mathrm{~S}, \mathrm{R} 3 \mathrm{~W}$

SE 1/4 Sec 20, T18S, R3W

$\checkmark \mathrm{NE} 1 / 4 \mathrm{Sec} 29$, T18S, R3W

$\checkmark \mathrm{SE} 1 / 4 \mathrm{Sec} 29$, T18S, R3W

$\checkmark \mathrm{NE} 1 / 4 \mathrm{Sec} 32, \mathrm{~T} 18 \mathrm{~S}, \mathrm{R} 3 \mathrm{~W}$

$\checkmark$ SE $1 / 4$ Sec 32, T18S, R3W

$\sim \mathrm{NE} 1 / 4 \mathrm{Sec} 5, \mathrm{~T} 19 \mathrm{~S}, \mathrm{R} 3 \mathrm{~W}$

- $\mathrm{SE} 1 / 4 \mathrm{Sec} 5$, T19S, R3W

$\nu$ SW $1 / 4$ Sec 5, T19S, R3W

$\checkmark$ NW 1/4 Sec 8, T19S, R3W

$\sim \mathrm{SW} 1 / 4 \mathrm{Sec} 8$, T19S, R3W

$r$ NW'1/4 Sec 17, T19S, R3W

i SW $1 / 4$ Sec 17, T19S, R3W

$\vee$ NW 1/4 Sec 20, T19S, R3W 


\section{GENTRAL WARRANTY DEED \\ trolkowing Kenvian stesutory Werrenty Rormu}

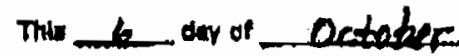
$-2002$

Jece $v$. Fogter and gera $x$. Boptex, hugband and wite

\section{CONYEY(O) ANO WARPANT(A) TO}

Potzer Dartax, a Kangas partnerohip

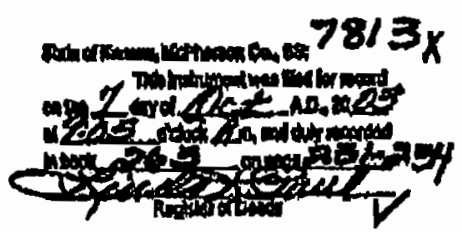

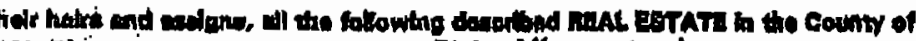
MePriersion stuts of Kentes, wowtes

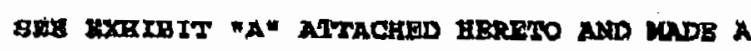

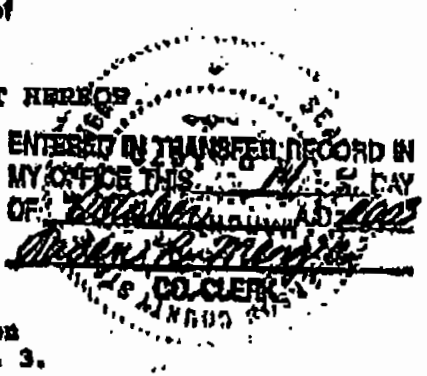

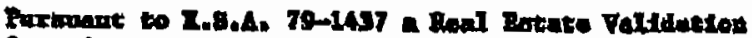
Quecteloninese is mot required due to comeptlon to. 3.

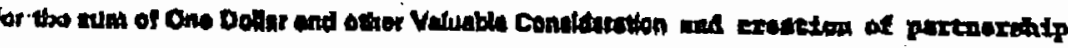
Xerm ANp suiver To:

kabemonta, Reptrfetions and Reservations of record, if any.
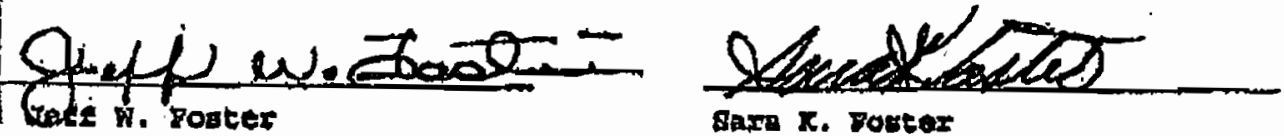

garn X. Fortex

Stati of ransaA Mophareon coum, as.

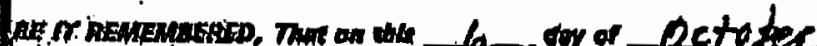

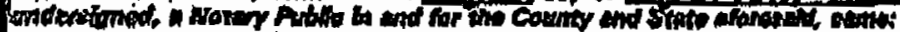

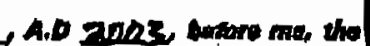

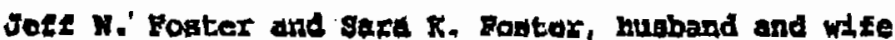

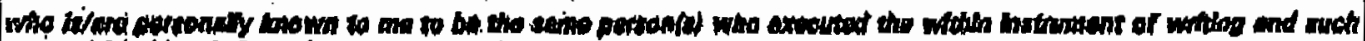

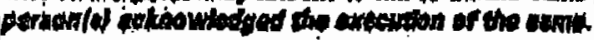

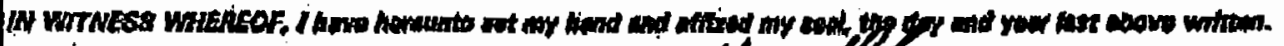

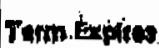

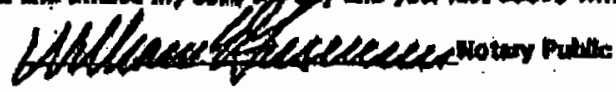




\section{EXHIBIT A}

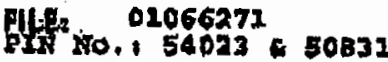

PARCEI I.

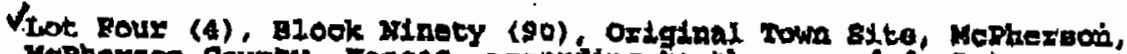

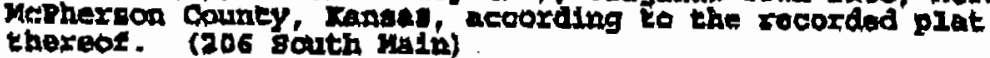

PिAR日I III

Ire

Hert 67 leat of Hots one (1) and wo (2) Blook Elghty (60), Oxiginal Town glte, Mepheraon, Mepheraon County, kanaas, according to the roooxdad plat theroof. (aII Regt Kangau)

PARCgr III,

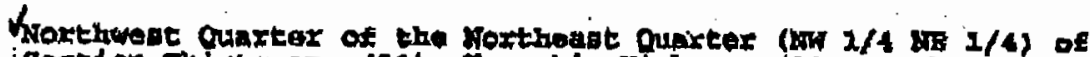
Gection Thixty-one (32), Tomshts 8ighteen (1B) gouth, Lange :Three (3) Weat of the Blsth Pridtelpal Marlalan, Mepharion County, Kanstus.

\section{AND}

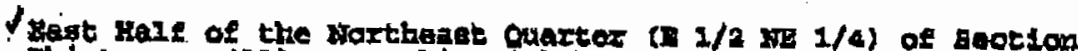

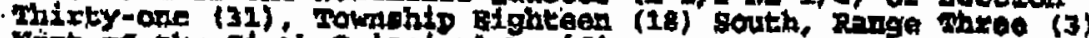

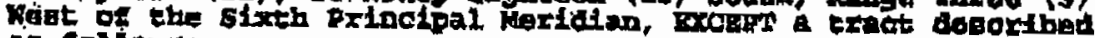
is toljowst

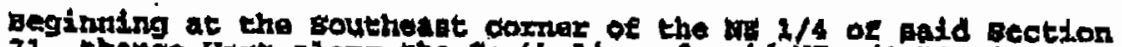

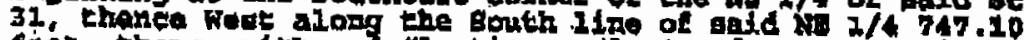

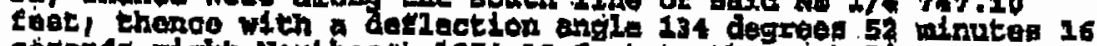
ajconds right-Northealt I054.18 feet to the Dist Ifre of and Nis 1/4, thende wet a detlection angle 134 degreas 52 minutes

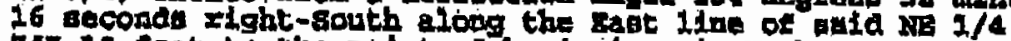
$7 \$ 7.10$ teet to the polyt of beglining, In Maphamon Cowuty. Kansas. frititon West)

BARCEL IV:

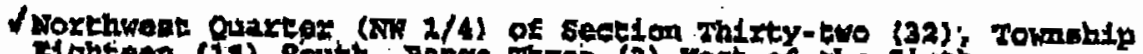
Iighteen. (18) Bouth, xange zhros (3) nest of the gixtb

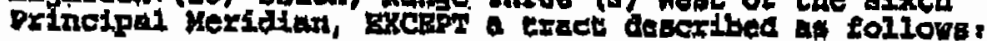

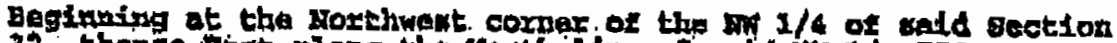

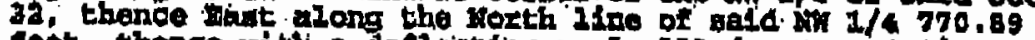

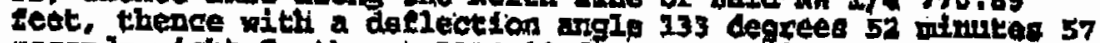
mocond: right-gonthwest I114.10 Eeet to a polnt on tho rast

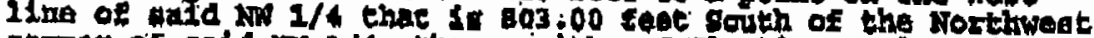

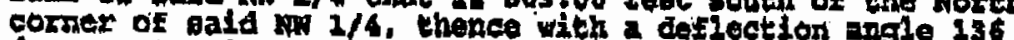

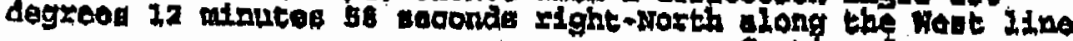
Cont Iruad om apxt page 01066971 


\section{EXHIET A}

FILE1 01066271

OF cald NW $1 / 4803.00$ seat to the polnt of beginning, 1n Mopherson County, Kancas. (R11tar Eut)

Pukcers vi

$\checkmark$ Bouthreat Quartar (BW 1/4) af section 81even (11), Torahtip N1ngtaon (19) 8outh, Range Bour (4) Regt of the Bixth Prdactpal

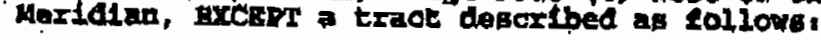

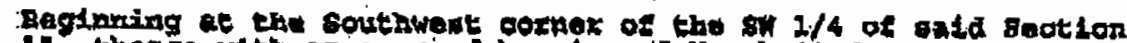

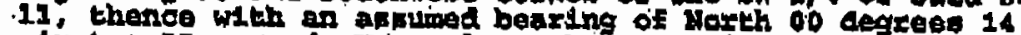

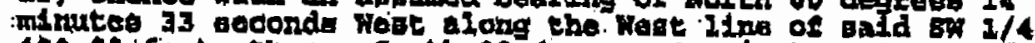
400.00 ficot, ehenca vouth 89 degreas 48 minutes 59 mecondy sact

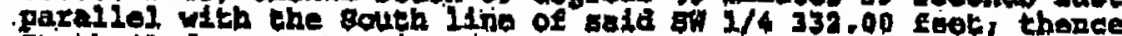

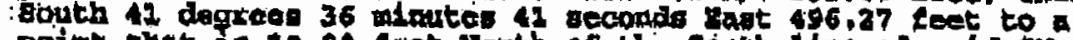
point ente in 30.00 eet Jorth of the South line of said bu 1/4; thenco sotith 00 degiees 14 minutis 33 eeconds gagt - paraliel with the Negt Itne of atd si 1/4 30.00 Eset to the

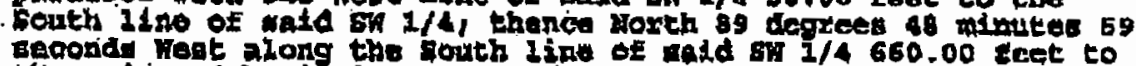
tho point of boglrintry, Nobnerion county, Kanaas. (Iubin)

PARCEL VI,

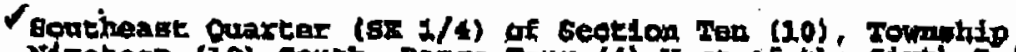
Himateen (19) Bouth, pange Four (4) Woet of the Sixth principal Nortalan, Mapherson' county, Kangas. (Nattrler)

DARCEL VII,

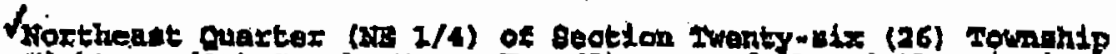
Eighteren (18) Bouth, lenge Two (2) Weat of the stxth pxinoipal

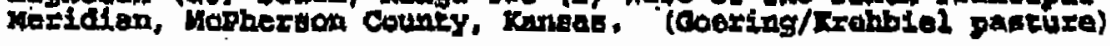

PARCEX viII:

$\checkmark$ southeart Quarter (as 1/4) of beotion Nonty-tour (21),

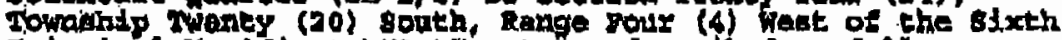

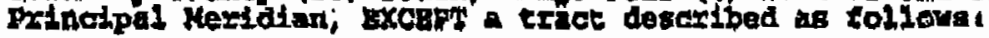
Commencing it the Bouthest corner of the ag $1 / 4$ of waid

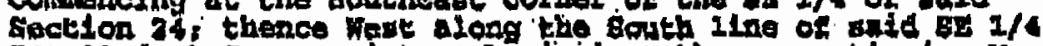

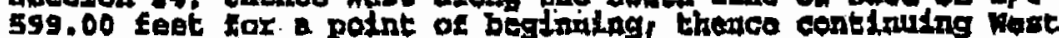

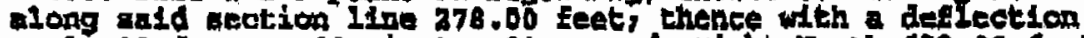
angle 90 degrees 00 molntes 00 beconds right-kdrth 623.00 loet: contimuod on next pase

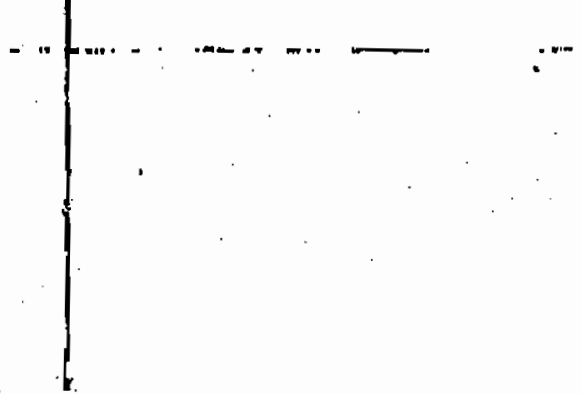




\section{EXHIBII A}

FiLG: 01066272

thence with a detlection angle 90 deajees 00 minutes 00 oeconds rigtut-trajt 270.00 faet, thande with deeseotion ang10 90

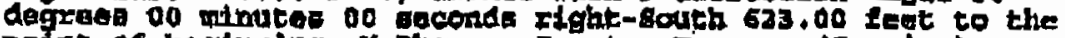
point of beginning, Mapherson county, Kunaas. (Eoering)

PARCAS IX,

21 of Grantors Interabie Interest in and tor

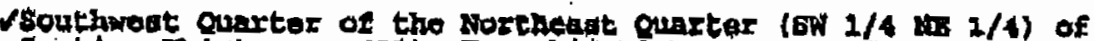

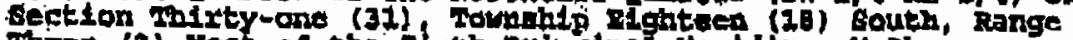
Three (3) vest of the Blxth Prinatpal Meridian, MePhoruon Cotrity, Kansan. (Jotudon 40) 


\section{NNHW: GI (O)T:TLAUNID)}

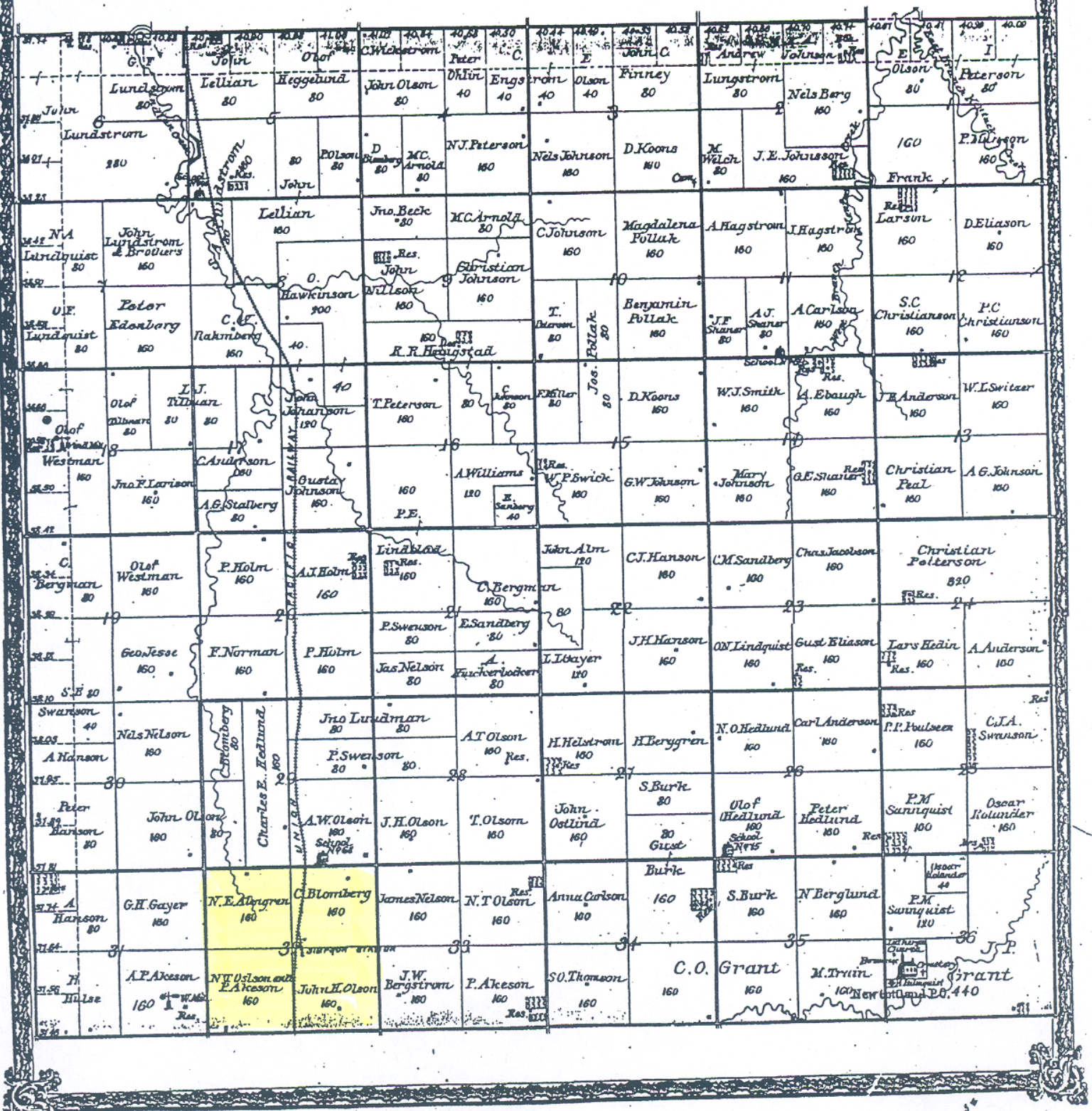




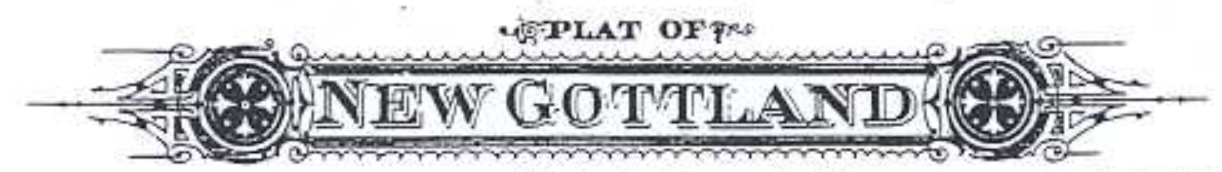

Scale 2 Inches to the Kile.

Township 18 South. Range 3 West.

of the 6th Peincipal Meridian

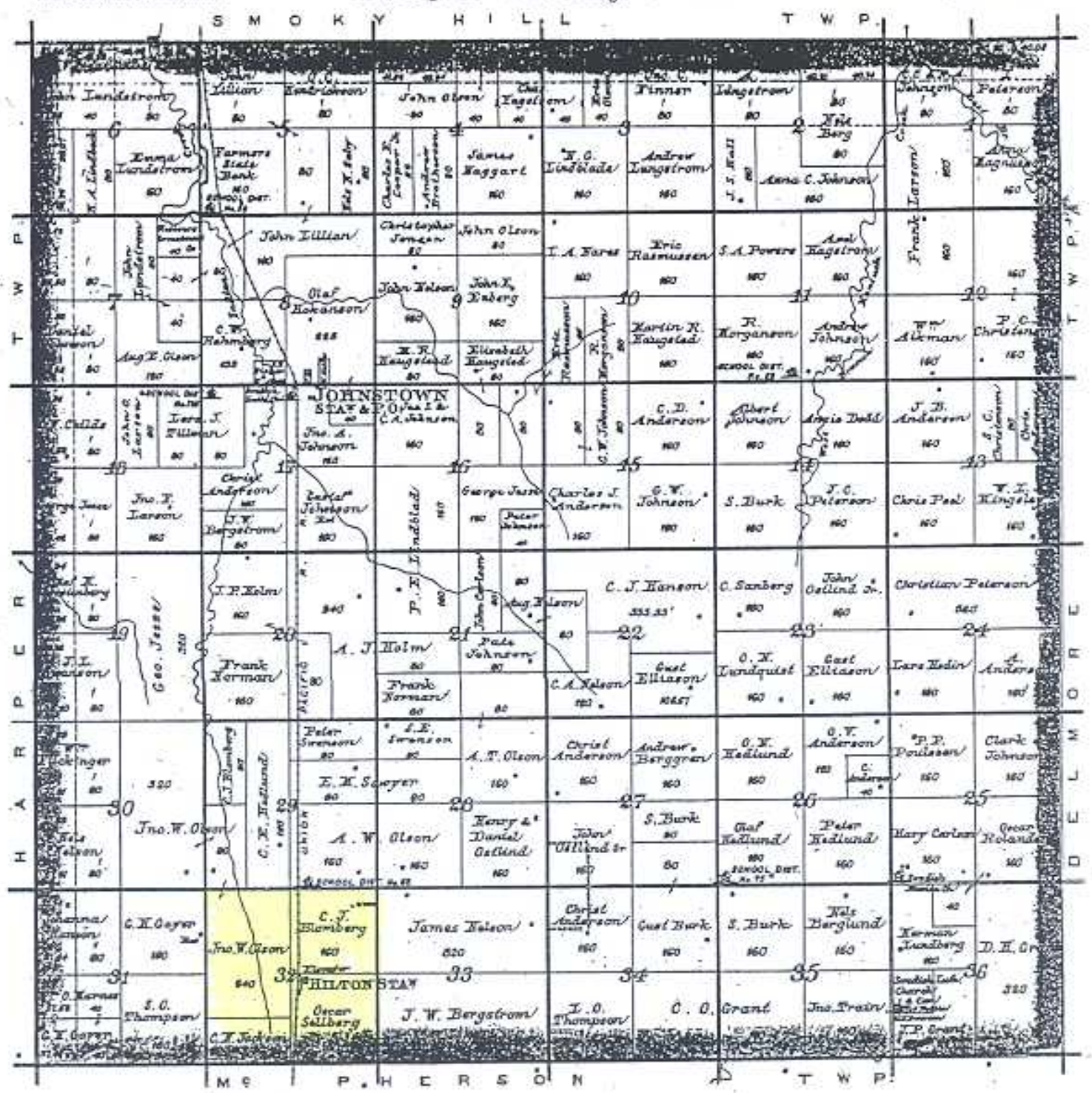




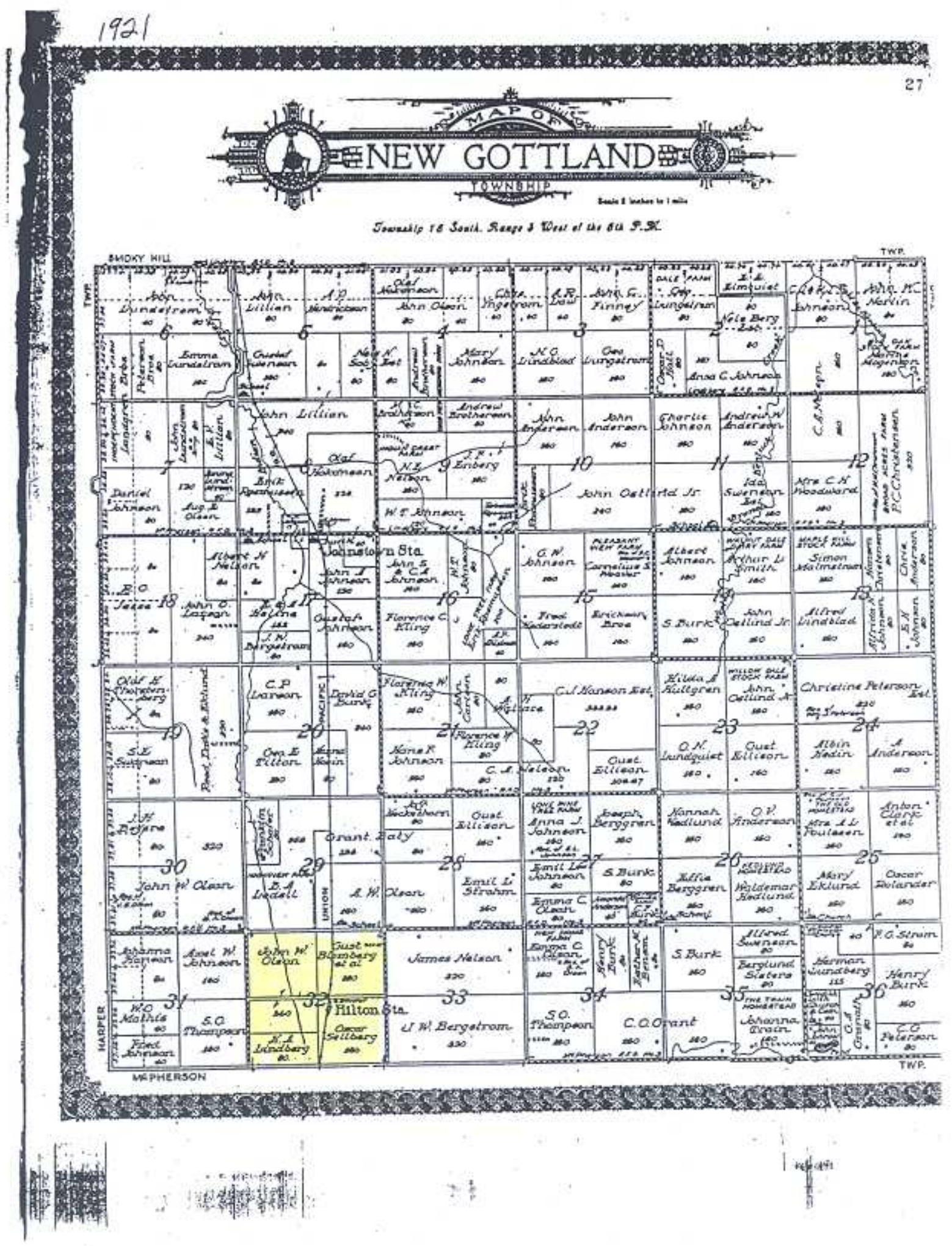




\section{NEW GOTTLAND}

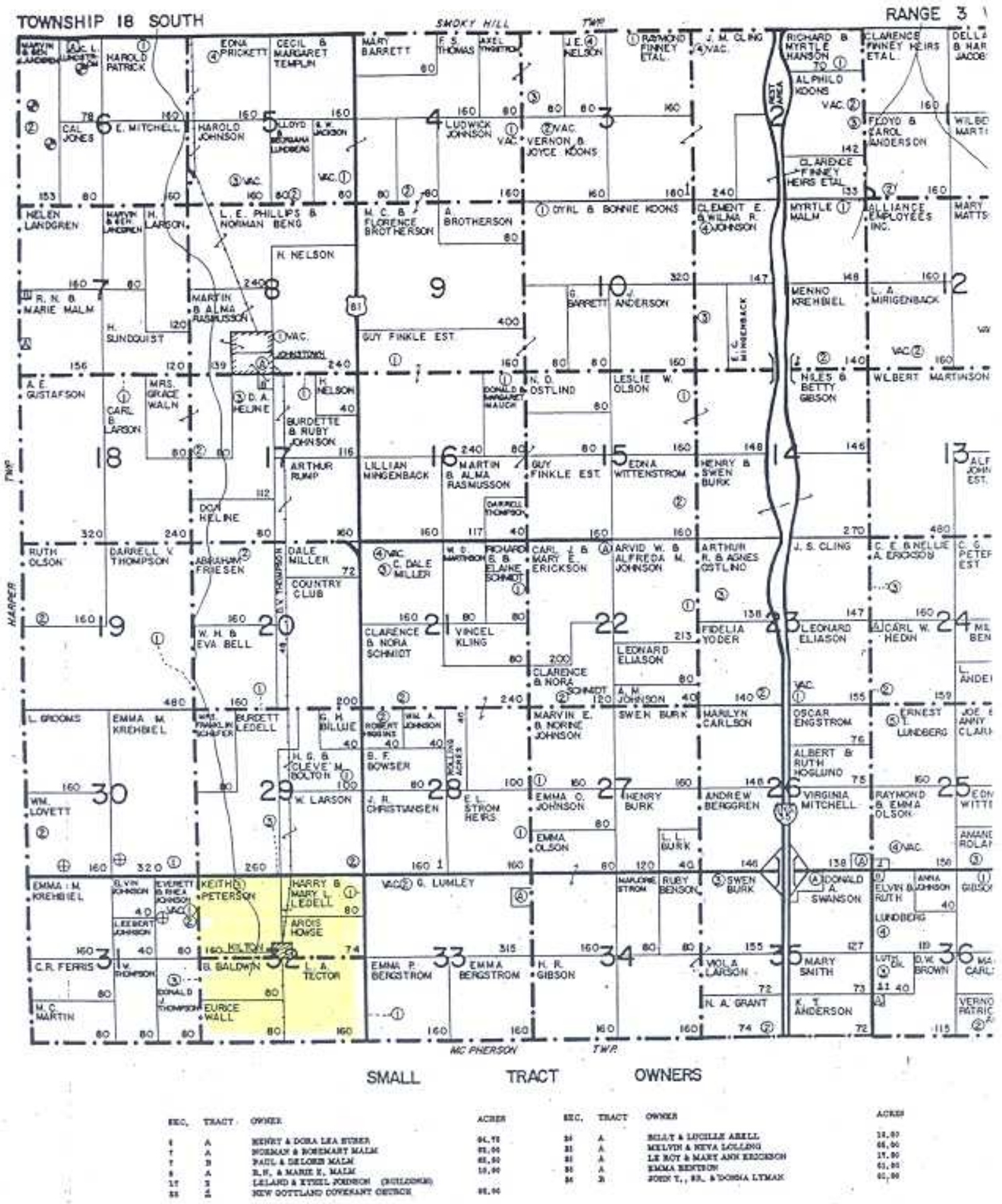




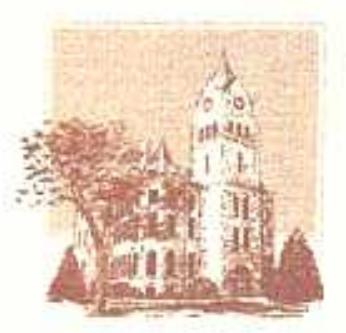

\section{MCPHERSON COUNTY}

MAPPING DEPARTMENT

Kovin Beakey

Cartographer?

P.O. Box 530

Mcipherson, KS 67460

690-24:- ह577

FoDX $=020,245-0005$

E-mail: hbeakey encphorsoncountyks-us - Wet: mophersoncountykshus

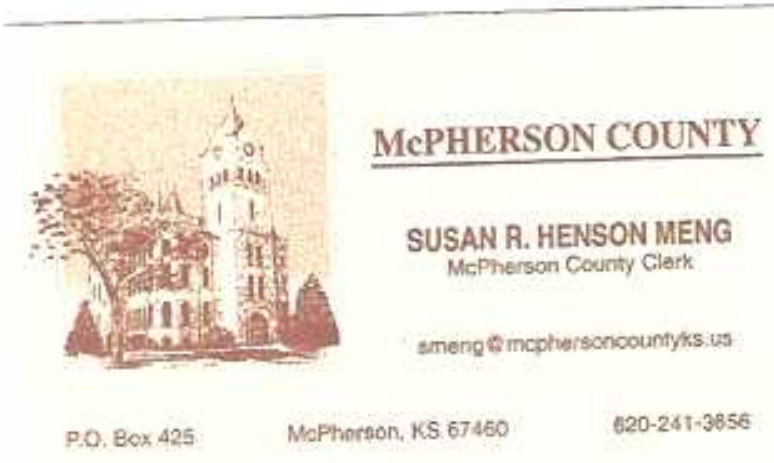

McPherson County

Government Website

\section{mephersoncountyks.us}

It's user friendly and very informational! 
MCPHERSON COUNTY, KANSAS

PARCEL IDENTIFICATION REPORT

THIS INFORMATION HAS BEEN COMPLLD FOR AD VALORUM TAXPURPOSES ONLY

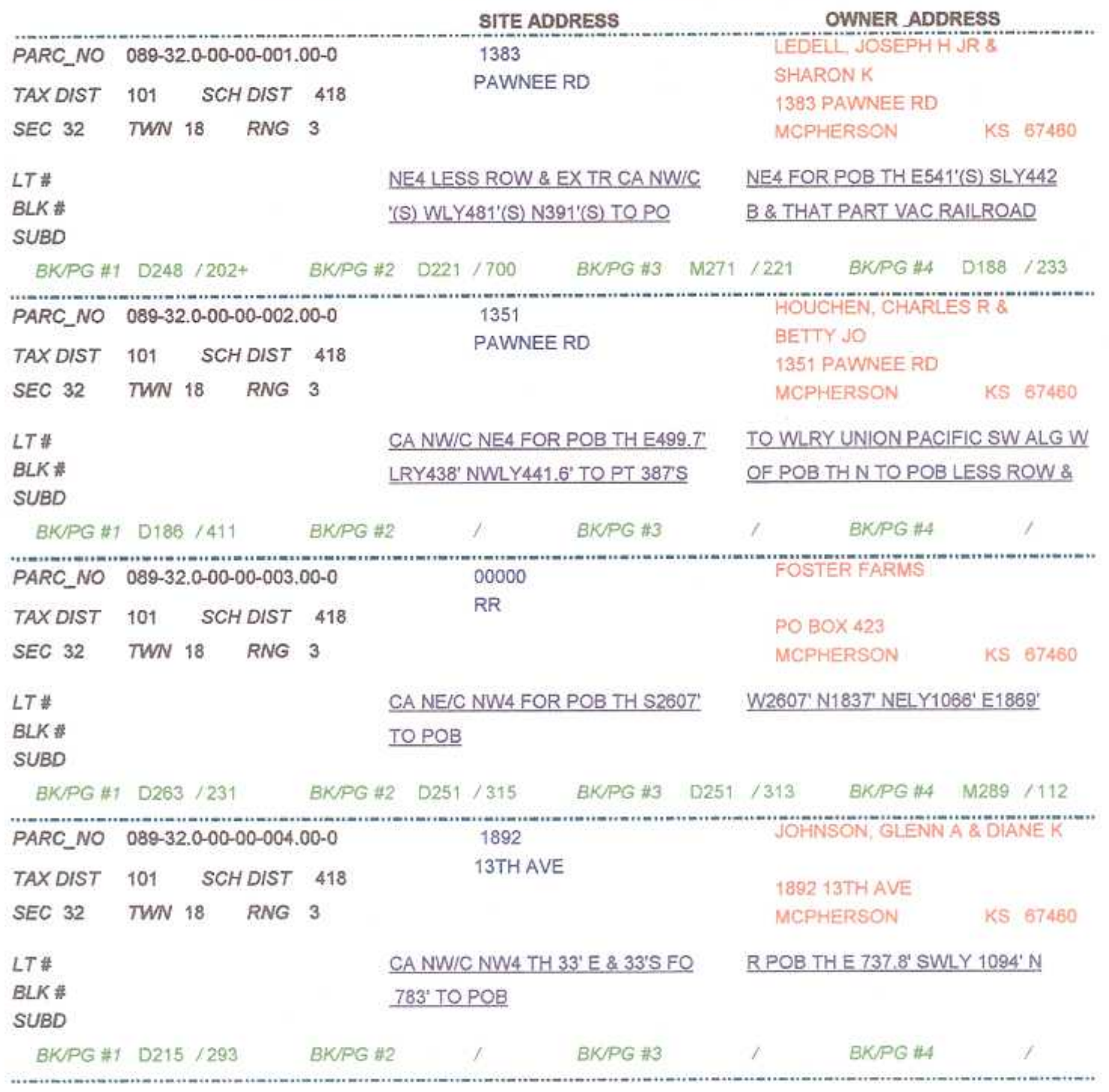

Fridำ A 


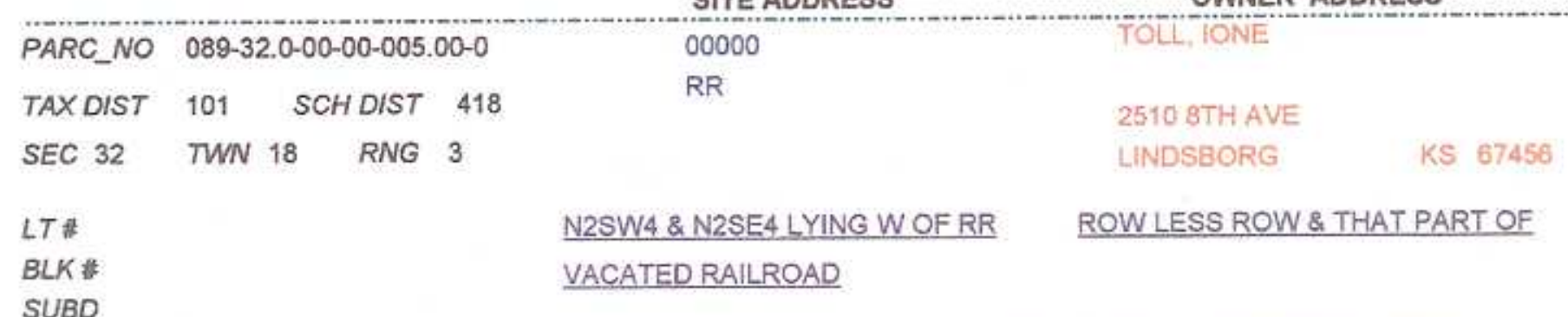

SUBD

BKJPG \#1 D603 /339 BK/PG \#2 D603/289 BK/PG\#3 D260/812 BK/PG \#4 D256/444

$\begin{array}{llllll}\text { PARC_NO } & 089-32.0-00-00-008.00-0 & 00000 & \text { DOSSETT, ELSIE FAMM TRUST } \\ \text { TAXDIST } & 101 \quad \text { SCHDIST } 418 & \text { RR } & \text { TTEE } \\ \text { SEC 32 } & \text { TWN } 18 \quad \text { RNG } 3 & & 1198 \text { LIMESTONE } & \text { KS } 67480\end{array}$

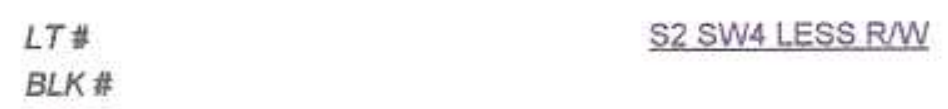

S2 SW4 LESS R/W

$B L K$ \#

SUBD

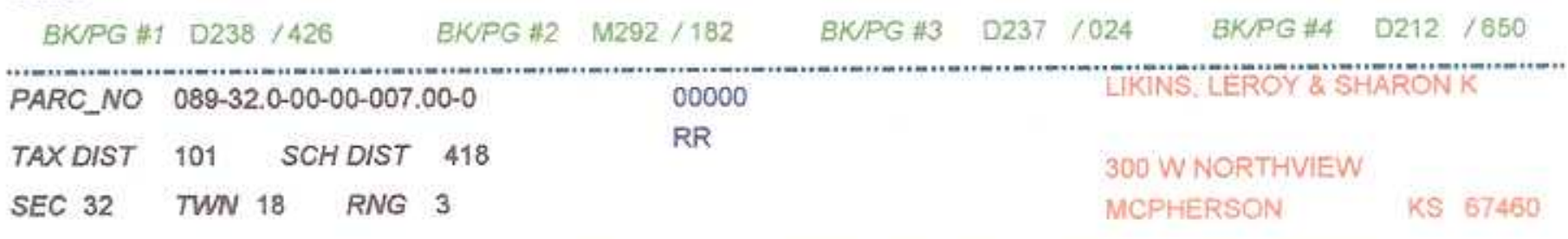

$L T \#$

S2SE4 LYING W OF RR ROW \& THAT PART OF VAC RAILROAD

BLK\#

SUBD

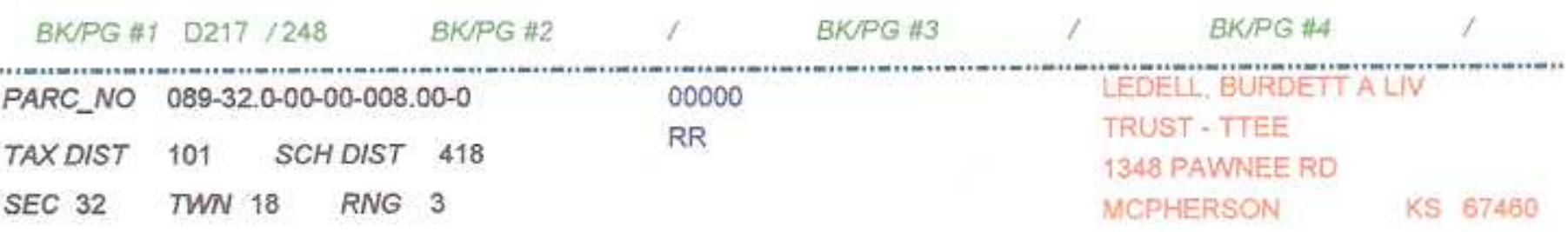

$L T \#$

BLK\#

SUBD
BKJPG \#1. D257/367
BK/PG H2 D246/486
BKVPG \#3 D246 /480

$\begin{array}{ll}\text { SE4 LYG E OF RR RIW EX TR DESC } & \text { AS FOL CA NW/C SE4 TH } 270^{\prime} E \text { \& } \\ 16.5^{\prime} \text { 'S FOR POB TH E33' SWLY660 } & \text { W33' NELY } 660^{\prime} \text { TO POB \& THAT }\end{array}$

$\begin{array}{llll}\text { PARC_NO } & 089-32.0-00-00-009.00-0 \\ \text { TAXDIST } & 101 & \text { SCHDIST } & 418 \\ \text { SEC } 32 & \text { TWN } 18 \quad \text { RNG } & 3 \\ \text { LT } & & & \\ \text { BLK \# } & & \end{array}$

\author{
00000 \\ RR \\ CA NW/C SE 4 TH $270^{\prime} E$ \& $16.5^{\prime} S$
NELY $860^{\prime}$ TO POB \& THAT PART OF \\ CA NW/C SE 4 TH $270^{\prime} E$ \& $16.5^{\prime} S$
NELYB6O' TO POB \& THAT PART OF \\ FOR POB TH E33' SWLY660' W33' \\ VAC RAILROAD \\ POBOXD \\ MOUNDRIDGE KS 87107 \\ MID KANASAS COOPPABSTN
}

BK/PG \#2 D206/187

BKN $\$ 3$

BKMPG \#4

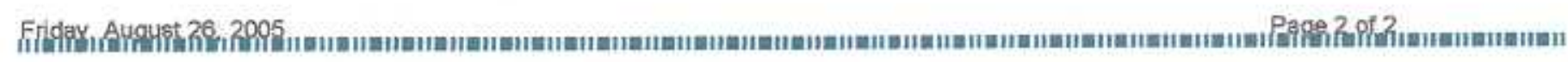




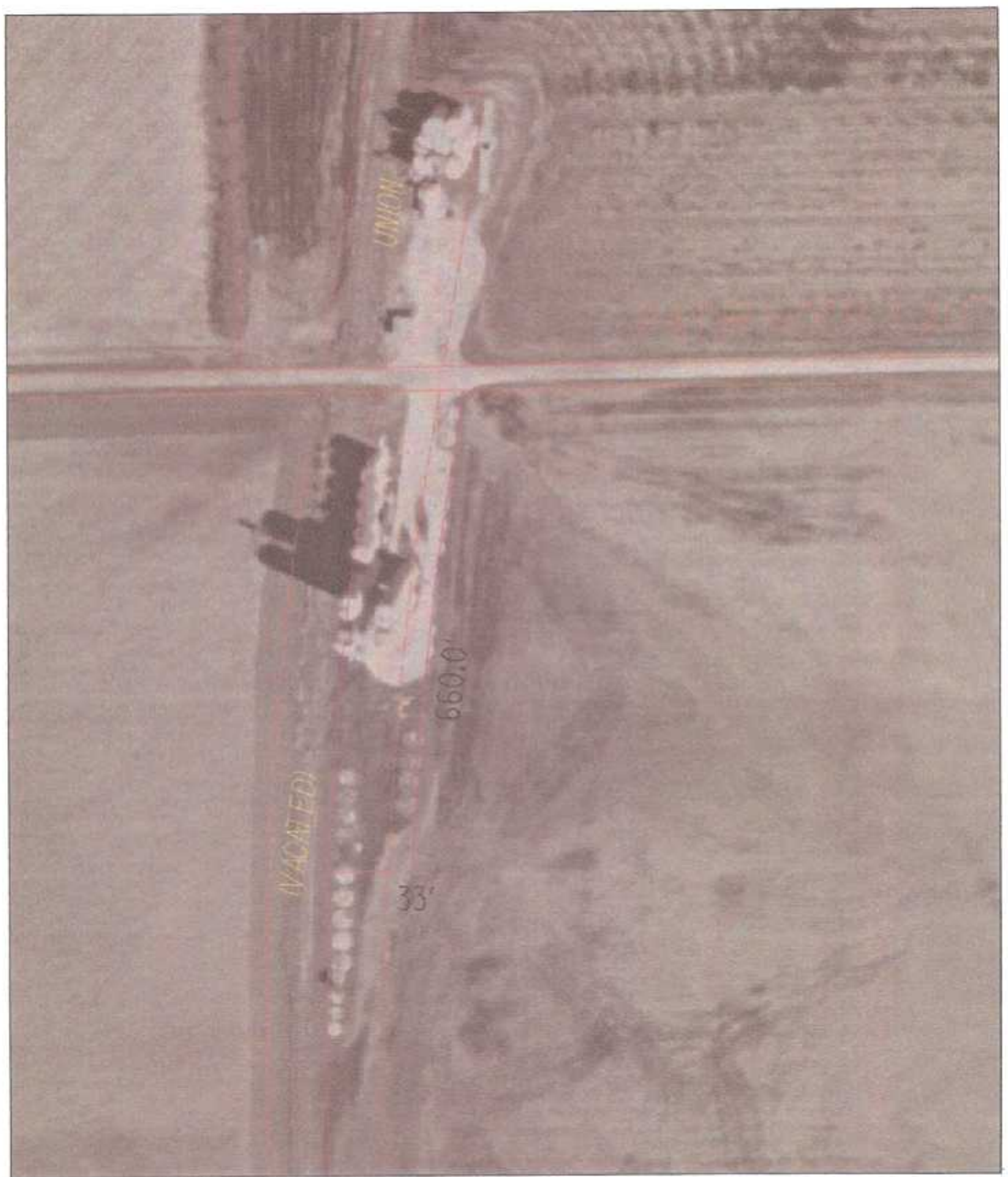




\section{Appendix C:}

\section{Waste Management Plan}




\section{Appendix C: Waste Management Plan}

This appendix presents the environmental protection controls and performance standards enacted to ensure that environmental investigation and remediation activities do not adversely affect public health or the environment. In addition, this section discusses the mechanisms for establishing site-specific controls for each location undergoing response activities.

This section addresses only environmental protection controls and standards, not the numerous controls and standards for protecting the health and safety of site workers and the general public and for assuring the quality and accuracy of data gathered. The latter are discussed in the Master Work Plan (Argonne 2002).

\section{C.1 The Comprehensive Environmental Response, Compensation, and Liability Act of 1980 and Applicable or Relevant and Appropriate Requirements}

The federal Comprehensive Environmental Response, Compensation, and Liability Act of 1980 (CERCLA or "Superfund") 1 is the primary driver for the work addressed at former CCC/USDA facilities. CERCLA authorizes both short-term removal (emergency response) actions and long-term remediation. CERCLA specifies that response and cleanup must assure protection of health and the environment. Further, on-site remedial actions must attain (or receive waivers for) federal environmental applicable or relevant and appropriate requirements (ARARs), more stringent state environmental ARARs, and state facility siting laws. ${ }^{2}$

Compliance with ARARs derived from other environmental laws is a crucial feature of CERCLA. The ARARs identified for each site addressed under CERCLA authority provide cleanup goals and guide response implementation. Factors considered in identifying site-specific ARARs include the chemicals present, the location, the physical features, and the actions under consideration as remedies.

142 USC (United States Code) 9601 et seq.

2 Section 121(d). 


\section{C.2 The National Oil and Hazardous Substances Pollution Contingency Plan and U.S. Environmental Protection Agency Policy}

The 1990 National Oil and Hazardous Substances Pollution Contingency Plan (NCP) 3 extended the requirement for compliance with ARARs to include not only long-term remedial actions, but also more immediate removal actions as practicable (considering the urgency of the situation). ${ }^{4}$ The NCP explicitly requires the identification of ARARs only when the response evaluation phase is reached (in the case of remediation activities, during the remedial investigation or feasibility study). ${ }^{5}$ As a matter of policy, the EPA has stated that treatment or disposal of investigation-derived wastes produced during response activities must ensure protection of human health and the environment and must, to the extent possible, comply with (or receive waivers for) federal and state ARARs.

\section{C.3 Procedure for Identifying Controls and Standards}

In numerous documents, the EPA has published guidance for the identification of sitespecific ARARs and the subsequent determination of environmental protection controls and performance standards from the ARARs. For this investigation, federal statutes, executive orders, federal regulations, state laws, state regulations, and local ordinances were all reviewed as potential sources of necessary, reasonable, and prudent controls and standards. State laws and regulations and local ordinances were considered only when they are legally enforceable, consistently enforced, and more stringent than the corresponding federal requirements. In addition to statutes and regulations directed at protection of the environment, state laws and regulations pertaining to the siting of certain facilities were also reviewed.

In general, applicable requirements have basic statutory or regulatory jurisdiction, whether an activity is conducted under CERCLA or another authority. The EPA guidance defines applicable requirements as "cleanup standards, standards of control, and other

3 Published in the Federal Register on March 8, 1990 (55 FR 8666) and codified in Part 300 of Title 40 of the Code of Federal Regulations (40 CFR 300).

4 See NCP, 40 CFR Section 300.415(j) (55 FR 8666, 8843) and Section 300.435(b)(2) (55 FR 8666, 8852) (March 8, 1990).

540 CFR 300.430(d)(3). 
substantive environmental protection requirements, criteria, or limitations promulgated under Federal or State law that specifically address a hazardous substance, pollutant, contaminant, remedial action, location, or other circumstance present at a CERCLA site." Relevant and appropriate requirements, though they are "not 'applicable' ... at a CERCLA site, address problems or situations sufficiently similar ... that their use is well suited to the particular site."

Additional standards and controls for response activities derived from guidance or advisories issued by federal or state agencies are not legally binding, but they are defined as requirements to be considered in the formal ARAR process. Such standards and controls were reviewed for their usefulness in developing operating procedures or best management practices that assure compliance with federal and state regulations and protection of public health and the environment in conjunction with response activities at former CCC/USDA facilities.

Environmental protection controls and standards are classified as follows:

- Chemical Specific. Chemical-specific (or ambient) controls and standards are health-based or risk-based standards issued for specific chemicals or chemical families. The anticipated chemical-specific requirements applicable to the generation, management, and ultimate disposal of wastes derived from site investigations would involve carbon tetrachloride and, at some locations, nitrates. (Even though the nitrate contamination that might be encountered is not attributable to CCC/USDA activities, the presence of such contamination will establish the applicability of nitrate-specific controls in the management and disposal of wastes.)

- Performance, Design, or Action Specific. Performance-, design-, or actionspecific requirements are applicable to the generation, management, and disposal of wastes associated with planned response activities.

- Location Specific. Location-specific restrictions are placed on concentrations of hazardous substances - or the conduct of activities involving those substances - solely because of the special or sensitive nature of the location involved. It is beyond the scope of this document to develop a comprehensive list of location-specific requirements. However, this document directs that a reconnaissance survey of each location be conducted before characterization 
activities begin. This survey is to identify the presence of environmentally sensitive areas that could be affected by the investigation and response. Such sensitive areas include faults displaced in Holocene time; 100-yr floodplains; salt dome formations; historic properties; critical habitats upon which endangered or threatened species depend; wetlands; wilderness areas; wildlife refuges; areas immediately adjacent to streams or rivers; areas affecting national, wild, scenic, or recreational rivers; wellhead protection areas; and other sensitive areas as defined by the EPA or state authorities. In addition, the survey is to identify all requirements imposed by federal or state siting statutes and regulations.

The KDHE is the authorized state agency for developing and implementing environmental protection programs. ${ }^{6}$ Most Kansas environmental control programs are authorized to operate in lieu of their federal counterparts.

The federal and state environmental protection controls and performance standards that are applicable or relevant and appropriate to the activities anticipated at former CCC/USDA facilities are identified and analyzed in Table D.1 (at the end of this appendix).

\section{C.4 Implementation of Controls and Standards}

The application of the standards and requirements identified in Table C.1 depends on the chosen techniques for the response action, the specific location where activities will be conducted, and the chemical constituency of the resulting waste at each site. Consequently, the controls ultimately applied will be determined during the response activities at each location.

6 Kansas Statutes Annotated (KSA), Chapter 75, Article 56, Statute 75-5601. 


\section{C.4.1 CERCLA Wastes and the Off-Site Rule}

CERCLA wastes are any hazardous substance, pollutant, or contaminant ${ }^{7}$ generated in the course of a response action. CERCLA wastes are subject to requirements of the Resource Conservation and Recovery Act (RCRA), Subtitle C, if they are listed or identified as hazardous waste or exhibit a characteristic of hazardous waste. Such wastes may be placed only in a facility operating in compliance with RCRA or other applicable federal or state requirements. Furthermore, CERCLA wastes may not be transferred to a land disposal facility that is releasing contaminants into the environment; releases from other waste management units must be controlled. The purpose is to avoid contributions to present or future environmental problems by CERCLA wastes from response actions authorized or funded under CERCLA. Such wastes are therefore directed to management units determined to be environmentally sound. ${ }^{8}$

The Off-Site Rule (OSR) ${ }^{9}$ regulates the off-site transfer of CERCLA wastes. The OSR establishes the following:

- Criteria and procedures for determining whether facilities are acceptable for the receipt of CERCLA wastes from response actions authorized or funded under CERCLA.

- Compliance and release criteria, along with a process for determining whether facilities are acceptable on the basis of those criteria.

The OSR applies to any remedial or removal action involving the off-site transfer of CERCLA waste, whether the action is conducted by the EPA, states, private parties, or other federal agencies, if the action is financed through the Superfund or is taken under CERCLA legal authority.

\footnotetext{
7 Section 121(d)(3).

8 Preamble to final Off-Site Rule (OSR), 58 FR 49200, 49201, September 22, 1993.

940 CFR 300.440.
} 


\section{C.4.2 Investigation-Derived Wastes}

Although the investigations conducted at former CCC/USDA facilities are generally minimally intrusive, they do produce small amounts of wastes. Investigation-derived waste (IDW) streams include (1) cuttings from soil borings and well installations, (2) well development fluids, (3) purge water recovered from sampling wells, (4) residuals and quality control duplicates from sample analyses performed both in the field and in off-site laboratories, (5) equipment decontamination rinsates, (6) soiled and potentially contaminated personal protective equipment, and (7) other miscellaneous wastes associated with well installation and sampling of various environmental media.

Site characterization activities might result in air emissions of particulate matter or other materials from drilling equipment. Therefore, air pollution laws and regulations can be ARARs.

In all instances, investigation-derived wastes are to be managed in a manner consistent with the applicable EPA Office of Solid Waste and Emergency Response directive. In addition, field investigators are to seek site-specific guidance and directives from the appropriate regulatory authorities, including federal, state, and local environmental or public health authorities.

The approach to management of IDWs is as follows:

- Leave a site in a condition no worse than before the project.

- Remove wastes that pose an immediate threat to human health or the environment.

- Keep on-site wastes that do not require off-site disposal or extended aboveground containerization.

- Comply with federal and state ARARs, to the extent practicable.

- Exercise careful planning and coordination for management of project-derived wastes. 
- Minimize the quantity of wastes generated.

The approach to handling and disposal of soil and water IDWs is as follows:

- Soil cores collected during sampling will be retained in core boxes for lithologic descriptions and research. The cores will be transported and stored at an Argonne facility.

- Soil IDW from drilling activities will be stored on-site in 55-gal drums or a roll-off dumpster. A representative sample will be collected and analyzed by a KDHE-certified laboratory.

- A Solid Waste Disposal Authorization will be obtained from the KDHE for disposal of soil in a permitted landfill. If analytical data indicate the soils cannot be disposed in a permitted landfill, alternative disposal methods will be proposed to KDHE for review.

- Water IDW will be stored on-site in 55-gal drums or polyurethane containers. If acceptable to the KDHE, the wastewater will be aerated prior to sampling. Then the wastewater will be analyzed for VOCs and nitrate.

- The results of the wastewater analyses will be discussed with the KDHE, and the appropriate disposal method will be determined. 
TABLE C.1 Potential applicable or relevant and appropriate requirements (ARARs) for former CCC/USDA facilities in Kansas. ${ }^{a}$

Title Citation ${ }^{b}$ Description

Federal Laws and Regulations

Comprehensive Environmental Response, Compensation, and Liability Act (CERCLA)

42 USC 9601 et seq.

National Oil and Hazardous

Substances Pollution

Contingency Plan (NCP)
40 CFR Part 300

responding to both oilspills and hazardous substance releases.

O ff-Site Rule establishes procedures for planning and im plem enting off-site response actions (40 CFR 300.440$)$.

Draft Guidance for Evaluating the Vapor Intrusion to Indoor Air Pathway from Groundwater and

Soils (Subsurface Vapor Intrusion Guidance)

67 FR 71169 (Nov. 29, 2002) The draft guidance is intended to be a screening tool to aid users in determining whether a vapor intrusion pathway is complete and, if so, whether the complete pathway poses an unacceptable risk to human health at cleanup sites. With a complete pathway, humans are exposed to vapors originating from site contamination. The draft guidance begins with simple and generally reasonably conservative screening approaches and gradually progresses toward a more complex assessment involving increasingly greater use of site-specific data. For sites determined to have an incomplete vapor intrusion pathway, further consideration of the current site situation generally should not be needed. For sites determined to have a complete pathway, guidance is provided to evaluate whether the pathway does or does not pose a potential significant risk to human health. The draft guidance is not intended to provide recommendations to delineate the extent of risk or eliminate the risk.
Applicable to releases into the environment of hazardous substances and pollutants or contaminants that may present an imminent and substantial danger to public health or the national welfare.

Applicable to response actions involving off-site transfers of

hazardous substances, pollutants, or contaminants.

\section{Suggested for use at National} Priorities List and Superfund Alternative Sites. 


Title Citation ${ }^{b}$ Description

Safe Drinking Water Act (SDWA)

(Public Health Service Act)

National Primary Drinking Wate Standards

42 USC 300(f) et seq

40 CFR Part 141, Subparts B and $\mathrm{G}$

National Secondary Drinking

Water Regulations (NSDWRs)

Maximum Contaminant Level Goals (MCLGs)

Standards for Owners and Operators of Public Water Supply Systems

Underground Injection Control Regulations

Sole-Source Aquifers
40 CFR Part 143

40 CFR Part 141, Subpart F

40 CFR Part 141

40 CFR Parts 144-148

40 CFR Part 149
Establishes maximum contaminant levels (MCLs), which are health-based standards for public water systems. The MCL for carbon tetrachloride is

$5 \mu \mathrm{g} / \mathrm{L}$. The MCL for chloroform (total trihalomethanes) is $100 \mu \mathrm{g} / \mathrm{L}$.

Establishes secondary maximum contaminant levels (SMCLs) for public water systems to protect the aesthetic quality of the water. The SMCLs are not federally enforceable but are included as guidelines for the states.

Establishes non-enforceable drinking water quality goals at levels of no known or anticipated adverse health effects, with an adequate margin of safety. The MCLG for carbon tetrachloride is zero.

Provides treatment requirements for public water supply systems (i.e., systems that serve at least 25 people or have at least 15 connections).

Provides for protection of underground sources of drinking water.

Prohibits activities, including drilling, in an area designated a sole-source aquifer without special permission of the U.S. Environmental Protection Agency (EPA).
Applicable to organic groundwater contamination in a sole-source aquifer.

Applicable if groundwater is a source of drinking water and if the NSDWRs have been adopted as enforceable standards by the state.

May be relevant and appropriate if a more stringent standard is required to protect human health and the environment.

Relevant and appropriate in the establishment of cleanup goals for groundwater contamination.

Applicable if a response alternative involves underground injection.

Applicable if the aquifer in the area is a sole-source aquifer. 
Clean Water Act (Federal Water Pollution Control Act)

Ambient Water Quality Criteria

40 CFR Part 131

National Pollutant Discharge Elimination System (NPDES)
Section 304(a) of the Clean Water Act (CWA) requires the EPA to publish and periodically update ambient water quality criteria (AWQCs). These criteria are to ". . . accurately reflect . . . the latest scientific knowledge ... on the kind and extent of al identifiable effects on health and welfare including,

but not limited to, plankton, fish, shellfish, wildlife, plant life ... which may be expected from the presence of pollutants in any body of water...." Water quality criteria developed under Section 304(a) are based solely on data and scientific judgments on the relationship between pollutant concentrations and environmental and human health effects. These recommended criteria provide guidance for states and tribes in adopting water quality standards under Section 303(c) of the CWA.

Establishes NPDES permit procedures, criteria, and standards governing the discharge of pollutants from any point source into waters of the United States (Sections 318, 402, and 405 of the CWA). Most storm water discharges require coverage by an NPDES permit.
Developed for some organic constituents in groundwater; may be relevant and appropriate.

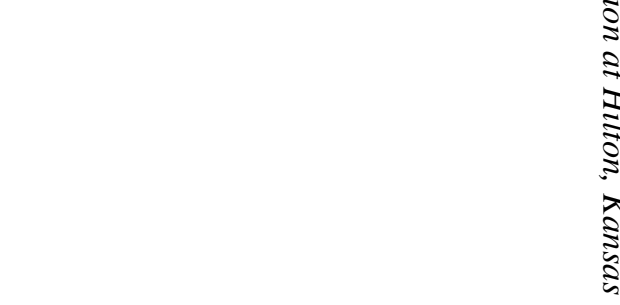

Discharge limits will be established if effluent is discharged to a surface water body.

No permit is required for on-site response actions under CERCLA, but the substantive requirements apply if a response alternative involves discharge into a creek or other surface water on-site. A permit is required if the discharge is to a creek or other surface water off-site. 
Dredge or Fill Requirements

40 CFR Parts $230-233$

(40 CFR Part 6.302(a))

Executive Order on Protection of Executive Order 11,990 Wetlands

\section{Rivers and Harbors Act of 1899}

\section{Section 10 Permit}

Executive Order on Floodplain Management

33 USC 401 et seq.

33 CFR Parts 320-330 (40 CFR Part 6.302(a))
Forms the foundation of the water-quality-based pollution control program mandated by the CWA. These standards define the goals for a water body by designating the water body's uses, setting criteria to protect those uses, and establishing provisions to protect water bodies from pollutants.

Provides general pretreatment standards and regulations for existing and new sources of pollution; establishes standards to control pollutants that pass through or interfere with treatment processes in publicly owned treatment works (POTW) or that may contaminate sewage sludge.

Requires permits for discharge of dredged or fill material into navigable waters.

Requires federal agencies to avoid, to the extent possible, adverse impacts associated with the destruction or loss of wetlands and to avoid support

of new construction in wetlands if a practicable alternative exists.

Executive Order No. 11,988
Requires permit for structures for work in or affecting navigable waters.

Requires federal agencies to evaluate the potential effects of actions in a floodplain to avoid, to the extent possible, the adverse impacts associated with direct and indirect development of a floodplain
Applicable to surface water discharges.

Applicable if a response alternative involves discharge to publicly owned treatment works.

Applicable if a response alternative requires discharge of dredged or fill material into navigable waters.

Applicable if a response alternative has a negative effect on a wetland.

Applicable if a response alternative affects a navigable waterway.

Applicable if activities are located in a 100-yr floodplain. 
National Emissions Standards for Hazardous Air Pollutants (NESHAPs)

\section{CFR Parts 61 and 63}

40 CFR Part 50

National Primary and Secondary Ambient Air Quality Standards

\section{Solid Waste Disposal Act (as} amended by the Resource

Conservation and Recovery Act

[RCRA])

Criteria for Classification of Solid

Waste Disposal Facilities and

Practices

40 USC 6901 et seq.
Establishes national primary and secondary ambient air quality standards under Section 109 of the Clean Air Act to protect public health and welfare.

Implements Section 112 of the Clean Air Act, which governs the federal control program for hazardous air pollutants (HAPs). Identifies emission standards for HAPs that originate from specific categories of sources, including site remediation. NESHAPs are technology based and are issued to limit the release of specified HAPs from specific industrial sectors. A Federal Register notice published for carbon tetrachloride (50 FR 32621 [August 13, 1985]) and chloroform (50 FR 39626 [September 27, 1985]) included consideration of serious health effects, such as cancer, due to ambient exposures.

\section{CFR Part 257}

Establishes classification criteria for sanitary landfills and open dumps, which are prohibited (Sections 4005 and 1008 of RCRA). At a minimum, facilities meet the sanitary landfill classification "only if there is no reasonable probability of adverse effects on health or the environment ..." [Section 4004(a) of RCRA].

Provides for protection of surface water and groundwater at solid waste disposal facilities (40 CFR 257.3-3 and -4).
Applicable if contaminants are discharged to the air during treatment.

Applicable if the identified HAPs are emitted from a specific source category (for example, if on-site treatment units with emissions are part of response actions).

Applicable if a response alternative involves land disposal of solid waste.
Applicable if a response action includes provisions for an on-site landfill. 


Title Citation ${ }^{b}$ Description

\section{Solid Waste Disposal Act (cont.)}

Criteria for Municipal Solid Waste Landfills

Identification and Listing of Hazardous Wastes

\section{Standards Applicable to} Generators of Hazardous Waste

Standards Applicable to

Transporters of Hazardous

Waste

Standards for Owners and

Operators of Hazardous Waste

Treatment, Storage, and

Disposal Facilities

Standards for Management of

Specific Hazardous Wastes and

Specific Types of Hazardous

Waste Management Facilities
40 CFR Part 258

40 CFR Part 261

40 CFR Part 262

40 CFR Part 263

40 CFR Part 264

40 CFR Part 266
Establishes minimum national criteria for municipal solid waste landfill units.

Identifies solid wastes that are subject to regulation as hazardous wastes under 40 CFR Parts 124, 262-265, 268, 270, and 271.

Establishes standards for generators of hazardous waste.

Establishes standards that apply to transporters of hazardous waste within the United States if the transportation requires a manifest under 40 CFR Part 262.

Establishes minimum national standards that define the acceptable management of hazardous wastes for owners and operators of facilities that treat, store, or dispose of hazardous waste.

Provides for groundwater protection standards, general monitoring requirements, corrective action requirements, and technical requirements.

Establishes requirements that apply to recyclable materials.
Applicable if municipal solid waste is placed in a municipal solid waste landfill.

Applicable if a material at the site is defined as a solid and hazardous waste; requires handling as a hazardous waste.

Applicable if hazardous wastes are generated as a result of on-site activities.

Applicable if a response alternative involves off-site transportation of hazardous wastes.

Applicable or relevant and appropriate if hazardous waste is disposed of on-site.

RCRA standards can be relevant and appropriate for groundwater at a site if an on-site landfill is constructed.

No substances are expected to be present at CCC/USDA sites in quantities to warrant recycling. 


\section{Hazardous Waste Permit}

Program

Universal Wastes

Technical Standards and Corrective Action Requirements

for Owners and Operators of

Underground Storage Tanks

Occupational Safety and Health Act (OSHA)

Occupational Safety and Health

Standards

Noise Control Act of 1972
40 CFR Part 268

40 CFR Part 270

40 CFR Part 273

40 CFR Part 280

29 USC 651 et seq.

29 CFR Part 1910

42 USC Sect. 4901 et seq
Establishes safety and health standards for workers. OSHA has set a limit of $100 \mathrm{ppm}$ for carbon tetrachloride in workplace air for an 8-hr work day, 40-hr work week.

Identifies hazardous wastes that are restricted from land disposal; defines limited circumstances under which an otherwise prohibited waste may continue

to be land disposed.

Establishes provisions covering basic EPA permitting requirements.

Establishes requirements governing universal wastes (hazardous waste batteries, hazardous waste pesticides that are either recalled or collected in waste pesticide collection programs, hazardous waste thermostats, and hazardous waste lamps).

Establishes regulations related to underground storage tanks.

Prohibits federal activities resulting in noise that would jeopardize the health or welfare of public.
Applicable or relevant and appropriate if hazardous waste are disposed of on-site.

No permit is required for on-site CERCLA response actions.

Substantive requirements are addressed in 40 CFR Part 264.

Applicable if universal wastes are generated or managed on-site in the course of investigation or response operations.

Applicable if a response alternative involves use of underground storage tanks.

Under 40 CFR Section 300.150 response actions under the NCP will comply with OSHA requirements for the safety and health of response action workers.

Applicable for activities such as drilling near a public access point. 
National Historic Preservation Act

16 USC 470 et seq.

Protection of Historic Properties

36 CFR Parts 800

(40 CFR 6.301(b))

Executive Order on Protection and Enhancement of Cultural Environments

\section{Executive Order on Preserve} America
Protects against the risks to life and property inherent in the transportation of hazardous material

by listing the materials deemed hazardous and describing required labeling, placarding, and

training. Hazardous materials are chemicals that the

U.S. Department of Transportation has determined

pose unreasonable risks to health, safety, and property during transportation activities.

Requires (Section 106) federal agencies to consider the effects of any federally assisted undertaking (including those carried out with federal financia assistance and those requiring a federal permit, license, or approval) on any district, site, building, structure, or object that is listed in the National Register of Historic Places or eligible for such listing; requires agencies to give the Advisory Council on Historic Preservation a reasonable opportunity to comment on those undertakings.

Defines how federal agencies meet the statutory responsibilities.

\section{Executive Order 11,593}

Executive Order 13,287

Requires federal agencies to preserve, restore, and maintain the nation's historic and cultural environment in their activities.

Formulates policy to promote intergovernmental
Applicable if an alternative involves transportation of hazardous

materials. Does not apply to on-site response operations.

Applicable if a district, site, building, structure, or object listed on or eligible for the National Register is on or adjacent to the site. cooperation and partnerships for the preservation and use of historic properties. 
Archaeological and Historic Preservation Act

Antiquities Act

Preservation of American Antiquities
16 USC 461 et seq.

(40 CFR 6.301(a))

16 USC 469 et seq (40 CFR 6.301(c))

16 USC 431 et seq.

43 CFR 3

(40 CFR Sect. 6.301(b))

Executive Order 11,593
Establishes a national policy to preserve historic sites and objects of national significance, including those located on refuges. Provides procedures for designation, acquisition, administration, and protection of such sites. Requires federal agencies to consider the existence and location of landmarks on the National Registry of Natural Landmarks and avoid undesirable impacts to such landmarks.

Carries out policy established by the Historic Sites Act. Establishes procedures for preservation of historical and archaeological data that might be lost or destroyed through alteration of terrain as a result of a federal construction project or a federally licensed activity or program. Directs federal agencies to notify the Secretary of the Interior whenever they find that a federal or federally assisted, licensed, or permitted project may cause loss or destruction of significant scientific prehistoric, or archaeological data.

Authorizes the President to designate as national monuments objects or areas of historic or scientific interest on lands owned or controlled by the United States. Requires a permit for examination of ruins, excavation of archaeological sites, and the gathering of objects of antiquity on lands under the jurisdiction of the Secretaries of Interior, Agriculture, and Army. Provides penalties for violations.

Protects all historic and prehistoric sites on federal lands and prohibits excavation or destruction of such antiquities without the permission (Antiquities Permit) of the secretary of the department that has the jurisdiction over those lands; authorizes the President to declare areas of public lands as national monuments and to reserve or accept private lands for that purpose.
Applicable if an entity on the National Register of National Landmarks is on or adjacent to the site.

Applicable if historical or archaeological data are on or adjacent to the site and if

construction projects or alteration of terrain at the site could destroy

historical or archaeological materials.

Applicable if site operations affect antiquities on federal lands. 
American Indian Religious Freedom Act

Executive Order on Indian Sacred Sites

Native American Grave Protection and Repatriation Act
43 CFR Part 7 ,

36 CFR Part 296

(40 CFR 6.301(b))

42 USC 1996 et seq.

Executive Order 13,007

25 USC 3001 et seq.
Supplements the provisions of the Antiquities Act of 1906. Establishes detailed requirements for issuance of permits for any excavation for or removal of archaeological resources from federal or Indian lands. Also established civil and criminal

penalties for the unauthorized excavation, removal, or damage of such resources.

Establishes uniform definitions, standards, and procedures to be followed by all federal land

managers in providing protection for archaeological resources located on public lands and Indian lands.

Protects and preserves the right of American Indians to have access to their sacred places: directs federal agencies to consult with Indian religious practitioners if a place of religious importance to American Indians may be affected by an undertaking.

Requires agencies managing federal lands to accommodate access to and ceremonial use of Indian sacred sites by Indian religious practitioners and to avoid adversely affecting the physical integrity of such sacred sites; also requires agencies to develop procedures for reasonable notification of proposed actions.

Establishes the priority for ownership or control of Native American cultural items excavated or discovered on federal or tribal lands after 1990 and the procedures for repatriation of items in federal possession; allows the intentional removal or excavation of Native American cultural items from federal or tribal lands only with a permit or upon consultation with the appropriate tribe.
Applicable if site operations affect archaeological resources on public or Indian lands.

Applicable if site operations affect access to sacred Indian sites.

Applicable if site operations affect access to sacred Indian sites.

Applicable if site operations involve excavation or discovery of Native American cultural items on federal or tribal lands. 


\section{Endangered Species Act}

Interagency Cooperation

\section{Migratory Bird Treaty Act}

\section{Migratory Bird Permits}

\section{Executive Order on}

Responsibilities of Federal

Agencies to Protect Migratory

Birds
43 CFR Part 10

16 USC 1531 et seq.

50 CFR Part 402

(40 CFR 6.302(h)

\section{USC 703 et seq.}

50 CFR Parts 10 and 21

Executive Order 13,186
Develops a systematic process for determining the rights of linear descendants and Indian tribes to certain Native American human remains, funerary objects, sacred objects, or objects of cultural patrimony. Defines items included under the Native American Grave Protection and Repatriation Act.

Describes the consultation procedure applicable to intentional excavation or inadvertent discovery of remains or objects covered.

Provides for the conservation of threatened and endangered plants and animals and the habitats in which they are found.

Requires action to conserve threatened and endangered species within critical habits upon which endangered species depend, including consultation and conferencing with the Department of the Interior (U.S. Fisheries and Wildlife Service/U.S. National Marine Fisheries Service).

Requires a permit from the U.S. Fisheries and Wildlife Service for the taking of protected migratory birds.

Directs federal agencies taking actions having or likely to have a negative effect on migratory bird populations to work with the U.S. Fisheries and Wildlife Service to develop an agreement to conserve those birds.
Applicable if threatened or endangered species or critical habitats are identified at the site. will affect a migratory pathway. 


Title Citation ${ }^{b}$ Description

Bald and Golden Eagle Protection 16 USC 668 et seq.

Act

\section{Eagle Permits}

50 CFR Parts 10 and 22

Fish and Wildlife Conservation Act

16 USC 2901 et seq.

Fish and Wildlife Coordination Act

16 USC 661 et seq (40 CFR 6.302(g))

\section{Wilderness Act}

Wilderness Preservation and Management

National Wildlife Refuge System Administration Act

Executive Order on the

Management and General Public

Use of the National Wildlife

Refuge System

\section{USC 1311 et seq.}

50 CFR Part 35, 43 CFR Part 6300

\section{USC 668dd}

Requires a permit from the U.S. Fisheries and

Directs preservation of a national network of lands

\section{wilderness areas and uses requiring authorization.} and waters for the conservation and management of fish, wildlife, and plant resources for the benefit of

present and future generations. Recognizes

compatible uses, while ensuring maintenance of biological integrity and environmental health. Wildlife Service to move nests because of construction or operation of project facilities.

Encourages states to develop conservation plans for nongame fish and wildlife of ecological, educational, aesthetic, cultural, recreational economic, or scientific value.

Requires consultation with the U.S. Fish and Wildlife Service when a federal department or agency proposes, authorizes, permits, or licenses any modification of any stream or other water body. Requires adequate provision for protection of fish and wildlife resources.

Applicable if project activities affect bald and golden eagle populations, including construction or operation of facilities that call for the moving of nests.

Applicable if significant populations are present at a site or are affected by site response activities.

Applicable if a response alternative will cause damage to or loss of wildlife by modifying a stream or body of water.

Describes the activities allowed and banned in

Applicable if a wilderness area exists on-site or adjacent to the site.
Applicable if a wildlife refuge area exists on-site or adjacent to the site 
TABLE C.1 (cont.)

Wild and Scenic Rivers

36 CFR Part 297

(40 CFR 6.302(e))

\section{Kansas State Laws}

Kansas Voluntary Cleanup and

Property Redevelopment Program
16 USC 1271 et seq.

Description

States that a federal agency may not assist, through grant, loan, license, or otherwise, the construction

of a water resources project that would have a

direct and adverse effect on the values for which a river in the National Wild and Scenic Rivers System

or a study river on the National Rivers Inventory was established (as determined by the Secretary of the Interior for rivers under the jurisdiction of the Department of the Interior and by the Secretary of

Agriculture for rivers under the jurisdiction of the Department of Agriculture).

Requires a notification process for federally assisted water resource projects in any portion of a designated river.

KSA, Chapter 65, Article 34

Sections 164 et seq. KAR, Title 28, Article 71
Applies to low- to medium-priority contaminated sites with minimal risk. Provides a mechanism for stakeholders (e.g., property owners, facility operators) cleaning up such sites to receive a "no further action" determination from the Kansas Department of Health and Environment (KDHE) (KAR 28-71-10). The KDHE is working with the to develop a Memorandum of Agreement providing voluntary participants with assurance of relief from future federal liability for the voluntary party's property.

Identifies remedial standards for cleanup of environmental media (KAR 28-71-11). Provides that groundwater cleanup levels will be based on the most beneficial use of the groundwater [KAR 28-71$11(1)]$
Applicable to on-site water resource projects located within, above, below, or outside a wild and scenic river or study river.

According to the KDHE, the program is truly voluntary and is designed for stakeholders to properly address contamination on eligible properties through private-state partnerships.

No additional burdens or

requirements are placed on voluntary participants. 
TABLE C.1 (cont.)

KSA, Chapter 65, Article 1, Sections 1,221 et seq.

KAR, Title 28, Article 73
Provides a way for landowners to achieve site closure by addressing environmental concerns caused by residual contamination.

An environmental use control is a restriction or prohibition of uses of or activities on a specific property, as requested by the property owner, to ensure future protection of public health and the environment when environmental contamination exceeding standards for unrestricted use remains on the property following appropriate assessment and/or remedial activities [KSA, 65-1,222(b)]. Eligible property is real property that exhibits environmental contamination exceeding standards for unrestricted use and is being or has been investigated or remediated, or both, in an approved program [KAR 28-73-1(c)].

Governs environmental use control agreements (KAR 28-73-3); long-term care agreements for properties that cover a large acreage, exhibit residual contamination characterized by higher toxicity or mobility, require complicated

maintenance or monitoring of protective structures, and require frequent or complicated site inspections (KAR 28-73-4); financial assurance for such

properties (KAR 28-73-5); duration of environmental controls (KAR 28-73-6); and restrictions,

prohibitions, and zoning in lieu of environmental use controls (KAR 28-73-7).
An environmental use control can be applied voluntarily to a property by the landowner to assure adequate protection of public health and the environment from contamination on the subject property. The protection offered can give a landowner relie from environmental liability concerns, making property more attractive to redevelopment or prospective buyers. 
Kansas Air Quality Control Act; Kansas Air Quality Regulations
KSA, Chapter 65, Article 30 KAR, Title 28, Article 19
Requires permitting and preconstruction notice for air contaminant sources. Provides for reporting and inspections. Establishes state emission standards for listed HAPs and state air quality standards to protect the public health.

Provides emission standards for HAPs (KAR 28-19 200 et seq.), including carbon tetrachloride and chloroform [KAR 28-19-201(a)]. Emission above a certain threshold requires an annual emissions fee (e.g., 10 tons/yr of a single HAP and 25 tons/yr of any combination of HAPS) (KAR 28-19-202).

Emissions above certain threshold amounts require a construction permit (e.g., 25 tons/yr of particulate matter [PM], 15 tons/yr of PM 10, 100 tons/yr of carbon monoxide, 40 tons/yr of volatile organic compounds [VOCs]) or a preconstruction approval (e.g., $5 \mathrm{lb} / \mathrm{hr}$ of PM, $2 \mathrm{lb} / \mathrm{hr}$ of PM 10, $50 \mathrm{lb}$ per 24-hr period of carbon monoxide, $50 \mathrm{lb}$ per 24-hr period of VOCs (either $15 \mathrm{lb}$ per 24-hr period or $3 \mathrm{lb} / \mathrm{hr}$ in a nonattainment area) (KAR 28-19-300).

Requires operating permits for certain stationary sources (28-19-500 et seq.).

Prohibits open burning (28-19-645 et seq.).

Establishes new source performance standards for municipal solid waste landfills, including reporting, calculation of nonmethane organic compound emissions, installation of collection and control systems (28-19-720 et seq.).

Establishes emission standards for major source HAPs (28-19-750 et seq.).
Applicable if any listed pollutants are discharged to the air during investigation or response through air stripping, thermal destruction, handling of contaminated soil, gaseous waste treatment, aeration,

or disposal in a municipal solid waste landfill. 
TABLE C.1 (cont.)

Water Pollution Control

Regulations
KSA, Chapter 65, Article 1; KAR, Title 28, Article 13

KSA, Chapter 65, Article 33; KAR, Title 28, Article 15a

KSA, Chapter 65, Article 33; KAR, Title 28, Article 16
Regulates the construction and use of underground storage reservoirs, disposal wells, and surface

ponds for the confinement, storage, and disposal of industrial fluids. Establishes approval and permitting requirements.

Establishes primary drinking water regulations. Adopts federal MCLs pertaining to public water supplies.

Provides for the maintenance and protection of public health and welfare and the use of surface water for aquatic life; for agricultural, domestic, and industrial water supply; and for recreation. Controls surface water use designation. Establishes surface water quality standards (KAR 28-16-28 et seq.)

Maximum contaminant level is defined as any of the enforceable standards for drinking water promulgated by the EPA [KAR 28-16-28b(aa)].

When the KDHE finds that the criteria are underprotective or overprotective for a given surface water segment, the KDHE may, in accordance with KAR 28-16-28f(f), make appropriate site-specific determinations (KAR 28$16-28 \mathrm{e}(\mathrm{a})$. Surface water must be free from the harmful effects of substances that produce any public health hazard; hazardous substances must not occur in surface water at concentrations that jeopardize public health and other protected life [KAR 28-16-28e(b)]
Applicable if a response alternative requires underground storage

reservoirs, disposal wells, or surface ponds.

Applicable if waste derived from investigation or response enters public water systems. Not applicable

to investigative wells that are not used for drinking water supply however, relevant and appropriate for establishing sampling and analysis parameters during an investigation.

Applicable if contaminated effluent from an investigative or response operation is discharged into surface water; also might be applicable to alluvial aquifers demonstrated to be hydraulically connected to surface water bodies. 
Water Pollution Control

Regulations (cont.)

Kansas Water Well Contractor's License Regulations; Water Well Construction and Abandonment Regulations

Kansas Water Appropriation Act; Kansas Water Appropriation Act Rules
Provides numeric criteria by use category for different parameters [KAR 28-16-28e(d)]. For chloroform the values are $28,900 \mu \mathrm{g} / \mathrm{L}$ for aquatic life-acute, $1,240 \mu \mathrm{g} / \mathrm{L}$ for aquatic life-chronic,

$15.7 \mu \mathrm{g} / \mathrm{L}$ for public health-food procurement, and $100 \mu \mathrm{g} / \mathrm{L}$ for public health-domestic water supply. (No values are specified for agriculture-livestock and agriculture-irrigation.) For carbon tetrachloride the values are $35,200 \mu \mathrm{g} / \mathrm{L}$ for aquatic life-acute, $6.94 \mu \mathrm{g} / \mathrm{L}$ for public health-food procurement, and $5 \mu \mathrm{g} / \mathrm{L}$ for public health-domestic water supply. (No values are specified for aquatic life-chronic, agriculture-livestock, and agriculture-irrigation.)
KSA, Chapter 82a, Article 12; KAR, Title 28, Article 30

KSA, Chapter 82a, Sections 701 et seq.

KAR, Title 5, Articles 1 et seq
Establishes procedures relating to the discharge of wastewaters under the NPDES program (KAR 2816-57 et seq.). National effluent standards are adopted by reference.

Requires water management planning for water quality management areas deemed critical by state authorities (KAR 28-16-69 et seq.).

Establishes limitations on the types of wastes that can be discharged to a POTW and governs pollutants from nondomestic sources that are subject to one or more pretreatment standards and that are indirectly discharged, or otherwise introduced by any means, into any POTW (KAR 2816-83 et seq.).

Applicable if investigation or response operations involve discharge into "waters of the state."

Applicable if contaminants resulting from investigation or response are discharged into a water quality management area.

Applicable if investigation or response alternatives involve discharge of contaminated groundwater to a POTW.

Establishes requirements for licensing of well drillers and standards for construction, operation, and plugging of wells (KAR 28-30-3 et seq.)

Applicable if investigation or response involves drilling of monitoring wells or other wells.

Establishes requirements for obtaining, maintaining, Applicable if water appropriations are and transferring water appropriations. 
TABLE C.1 (cont.)

Hazardous Waste Management Standards and Regulations
KSA, Chapter 65, Article 1; KAR, Title 28, Article 46

KSA, Chapter 65, Article 34 KAR, Title 28, Article 29

KSA, Chapter 65, Article 34 KAR, Title 28, Article 31
Governs discharges into underground injection wells (KAR 28-46-1 et seq.). In general, federal SDWA regulations are adopted by reference.

Establishes standards for management activities and facilities relative to solid wastes (KAR 28-29-21 et seq.).

Establishes location restrictions, design standards, operating standards, groundwater monitoring, corrective action, and financial assurance for closure and postclosure, for municipal solid waste landfill units (KAR 28-29-100 et seq.). Liners for new municipal solid waste landfills must be designed in accordance with KAR 28-19-

104(e)(1)(A)or(B). Alternative designs must demonstrate that a carbon tetrachloride concentration of $0.005 \mathrm{mg} / \mathrm{L}$ will not be exceeded [KAR 28-29-104(e)(1)(B)]. (Small units may request exemption [KAR 28-19-103].) Requires detection and assessment monitoring for carbon tetrachloride and chloroform (KAR 28-29-113).

Requires generators of solid waste to make a hazardous waste determination. For a waste that is not excluded from hazardous waste regulations and not listed as a hazardous waste, the determination is generally made through testing by a laboratory certified by the KDHE for such analyses [KAR 2831-4(b)].
Applicable if investigative or response wastes are introduced into wells for discharge or disposal.

Applicable if nonhazardous materials discarded as a result of the investigation or response are landfilled on-site.

Applicable if a municipal solid waste landfill is used.

\section{Applicable if investigation or} response operations generate solid wastes. 
Kansas Storage Tanks Act

Kansas State Board of Technical Professions

\section{Kansas Nongame and}

Endangered Species Conservation

Act; Kansas Nongame and

Endangered Species Conservation

Regulations
Establishes standards for hazardous waste generators and transporters and for storage, treatment, and disposal facilities (KAR 28-31-4 et seq.). Storage by certain hazardous waste generators for more than 90 days requires a permit [KAR 28-31-4(g)]. Adopts by reference federal land disposal restrictions (KAR 28-31-14).

Adopts by reference federal regulations governing universal wastes (KAR 28-31-15).

Governs storage tanks.

KSA, Chapter 74 , Article 70 KAR, Title 66, Number 66-6-1 through Number 66-14-12

KSA, Chapter 32, Article 9; KAR, Title 115, Article 15 land surveyors, geologists, and others. well as nongame species in need of conservation. Requires consultation with the Kansas Department of Wildlife and Parks regarding actions that might affect listed species and their critical habitats. Any

\section{Hazardous Waste Management
Standards and Regulations (cont.)}

Applicable to investigation samples or response-generated wastes that are determined to be hazardous and are transported or managed on-site through treatment, storage, and disposal. Transportation

requirements do not include a manifesting requirement for samples sent for characterization.

Applicable if universal wastes are generated or managed on-site in the course of investigation or response operations.

Applicable if a response alternative involves use of storage tanks to contain an accumulation of regulated substances; applies to associated piping, ancillary equipment, and the containment system.

Establishes requirements for licensing of engineers, Applicable if the services of engineers, land surveyors, and geologists are required for site investigation and response activities.

Designates endangered and threatened species, as projects that affect listed species or their habitat and that are publicly funded, are state or federally assisted, or require a permit from another state or federal agency require review and action permits (KSA 32-957 through 32-963, 32-1009 through 321012, 32-1033; KAR 115-15-1, -2, -3, and -4).
Applicable if threatened or endangered species are identified at or near the site. 
TABLE C.1 (cont.)

Kansas Unmarked Burial Sites Preservation Act
KSA, Chapter 24, Article 1; KAR, Title 5, Article 45

KSA, Chapter 75, Article 27 KAR, Title 118, Article 3

KSA Chapter 75, Article 27; KAR Title 126, Article 1
Requires prior approval of chief engineer before construction of floodplain fills and levees (KSA 24126)

Floodplain fill is material, usually soil, rock, or rubble, placed in a floodplain to an average height of more than $1 \mathrm{ft}$ above the existing ground, which has the effect of diverting, restricting, or raising the level of floodwaters of a stream (KAR 5-45-1).

Provides for protection and preservation of sites and buildings listed on state or federal historic registries (KSA 75-2715 through 75-2726; KAR 118-3-1 through 118-3-16).

Establishes Burial Sites Preservation Board. Prohibits unauthorized disturbance. Requires permits for excavation of any unmarked burial site, registered or unregistered (KSA 75-2741 through 75-2754; KAR 126-1-1 through 126-1-2).
Applicable if site activities involve construction of floodplain fills.

Applicable if the investigation or response site is a listed state or federal historic site or is adjacent to such a site and if activities requiring permitting are initiated.

Applicable if investigation or remediation activities encounter a burial site. 
a In addition to the ARARs listed here, the following advisory levels are to be considered:

- EPA Health Advisories (2004 Edition)

\begin{tabular}{|c|c|c|}
\hline Exposure & $\begin{array}{c}\text { Carbon } \\
\text { Tetrachloride } \\
\end{array}$ & $\underline{\text { Chloroform }}$ \\
\hline One day (mg/L) (10-kg child) & 4.0 & 4.0 \\
\hline Ten days (mg/L) (10-kg child) & 0.2 & 4.0 \\
\hline Reference dose (mg/kg day) & 0.0007 & 0.01 \\
\hline Drinking water equivalent level $(\mathrm{mg} / \mathrm{L})$ & 0.03 & 0.4 \\
\hline Lifetime (mg/L) (70-kg adult) & Not available & 0.7 \\
\hline
\end{tabular}

- EPA Region III Risk-Based Concentration Table (2006 Update) (carcinogenic effects)

Carbon

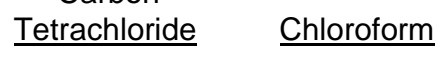

$\begin{array}{lll}\text { Tap water }(\mu \mathrm{g} / \mathrm{L}) & 1.6 \mathrm{E}-01 & 1.5 \mathrm{E}-01 \\ \text { Ambient air }\left(\mu \mathrm{g} / \mathrm{m}^{3}\right) & 1.2 \mathrm{E}-01 & 7.7 \mathrm{E}-02 \\ \text { Fish }(\mathrm{mg} / \mathrm{kg}) & 2.4 \mathrm{E}-02 & 1.4 \mathrm{E}+01 \\ \text { Soil, industrial }(\mathrm{mg} / \mathrm{kg}) & 2.2 \mathrm{E}+01 & 1.0 \mathrm{E}+04 \\ \text { Soil, residential }(\mathrm{mg} / \mathrm{kg}) & 4.9 \mathrm{E}+00 & 7.8 \mathrm{E}+02\end{array}$

b Abbreviations for citations:

CFR, Code of Federal Regulations

FR, Federal Register

KAR, Kansas Administrative Regulations

KSA, Kansas Statutes Annotated

Sect., Section

USC, United States Code 
Argonne

Environmental Science Division

Argonne National Laboratory

9700 South Cass Avenue, Bldg. 203

Argonne, IL 60439-4843

www.anl.gov

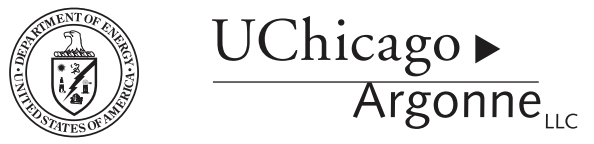

A U.S. Department of Energy laboratory

managed by UChicago Argonne, LLC 